\title{
Chemo-enzymatic synthesis of a tetra- and octasaccharide fragment of the capsular polysaccharide of Streptococcus pneumoniae type 14
}

\author{
John A.F. Joosten, Johannis P. Kamerling,* Johannes F.G. Vliegenthart \\ Bijvoet Center, Department of Bio-Organic Chemistry, Section of Glycoscience and Biocatalysis, Utrecht University, Padualaan 8, NL-3584 CH \\ Utrecht, The Netherlands
}

Received 16 April 2003; accepted 17 June 2003

\begin{abstract}
The chemo-enzymatic synthesis is described of tetrasaccharide $\beta$-D-Gal $p-(1 \rightarrow 4)-\beta-\mathrm{D}-\mathrm{Glc} p-(1 \rightarrow 6)-[\beta-\mathrm{D}-\mathrm{Gal} p-(1 \rightarrow 4)]-\beta-\mathrm{D}-$ Glc $p$ NAc- $\left(1 \rightarrow \mathrm{O}\left(\mathrm{CH}_{2}\right)_{6} \mathrm{NH}_{2}(\mathbf{1})\right.$ and octasaccharide $\beta$-D-Gal $p-(1 \rightarrow 4)-\beta-\mathrm{D}-\mathrm{Glc} p-(1 \rightarrow 6)-[\beta-\mathrm{D}-\mathrm{Gal} p-(1 \rightarrow 4)]-\beta-\mathrm{D}-\mathrm{Glc} p \mathrm{NAc}-(1 \rightarrow 3)-$ $\beta$-D-Gal $p-(1 \rightarrow 4)-\beta-\mathrm{D}-\mathrm{Glc} p-(1 \rightarrow 6)-[\beta-\mathrm{D}-\mathrm{Gal} p-(1 \rightarrow 4)]-\beta-\mathrm{D}-\mathrm{Glc} p$ NAc- $\left(1 \rightarrow \mathrm{O}\left(\mathrm{CH}_{2}\right)_{6} \mathrm{NH}_{2}(2)\right.$, representing one and two tetrasaccharide repeating units of Streptococcus pneumoniae serotype 14 capsular polysaccharide. In a chemical approach, the intermediate linear trisaccharide 3 and hexasaccharide 4 were synthesized. Galactose residues were $\beta$ - $(1 \rightarrow 4)$-connected to the internal $N$-acetyl- $\beta$ D-glucosamine residues by using bovine milk $\beta$-1,4-galactosyltransferase. Both title oligosaccharides will be conjugated to carrier proteins to be tested as potential vaccines in animal models.
\end{abstract}

(C) 2003 Elsevier Ltd. All rights reserved.

Keywords: Carbohydrates; Streptococcus pneumoniae; Oligosaccharide synthesis

\section{Introduction}

Gram-positive Streptococcus pneumoniae are members of the normal microflora of the human nasopharynx. Carriage of $S$. pneumoniae is more common in young children than in adults, and varies by geographic region and time. ${ }^{1,2} S$. pneumoniae is still a leading cause of lifethreatening diseases such as otitis media, pneumonia, meningitis, bacteraemia, and septicaemia, ${ }^{3,4}$ mainly due to a growing resistance towards antibiotics. ${ }^{5,6}$ Vaccination with the available 23 -valent capsular polysaccharide (CPS) vaccines ${ }^{7}$ offers protection for healthy adults to invasive pneumococcal diseases. ${ }^{8}$ However, these vaccines are ineffective in the most important high-risk groups, such as infants, small children, immuno-compromised patients, and the elderly, ${ }^{9}$ because they do not respond adequately to the T-cell independent polysaccharides as antigens. ${ }^{10}$ Conjugation of $S$. pneumoniae carbohydrate antigens to a protein carrier results in a $\mathrm{T}$ -

\footnotetext{
* Corresponding author. Tel.: +31-30-2533479; fax: +3130-2540980.

E-mail address: j.p.kamerling@chem.uu.nl (J.P. Kamerling).
}

cell dependent neoglycoconjugate antigen of which it has been proven that it gives an efficient immune response in the high-risk groups. ${ }^{11}$ Currently, neoglycoconjugate vaccines against $S$. pneumoniae serotypes, prepared by conjugation of isolated CPSs or of a mixture of polysaccharide-derived oligosaccharides to a protein carrier, have been introduced. ${ }^{11}$ The presence of oligosaccharide mixtures can complicate the product analysis, especially in the case of pneumococcal conjugate vaccines where many serotypes have to be included.

By using well-defined oligosaccharides for conjugation, it is possible to investigate the influence of different parameters originating from the carbohydrate part of the neoglycoproteins on their immunogenicity. Studies with oligosaccharide-protein conjugates related to the CPS of S. pneumoniae serotypes 3 and 6B showed that, via this approach, the minimal size of the carbohydrate antigen could be determined. ${ }^{12,13}$

Recently, we have reported the chemo-enzymatic synthesis of (spacered mimics of) fragments of the CPS of $S$. pneumoniae type 14 , comprising a branched tetrasaccharide and alkyl-bridged hexa- and octasaccharides. ${ }^{14,15}$ These oligosaccharides, together with 
tetra- and octasaccharide fragments of the type 14 CPS obtained by partial $\mathrm{N}$-deacetylation/deamination, were investigated for their immunological behavior. ${ }^{16}$ Based on the promising results of the branched tetrasaccharide, representing one repeating unit, it was decided to synthesize longer intact oligosaccharide fragments of the type 14 CPS. So far, we have reported the synthesis of 6aminohexyl-spacered penta- and hexasaccharide fragments, ${ }^{17}$ and here we report the chemo-enzymatic synthesis of 6-aminohexyl-spacered tetra- and octasaccharide fragments of the CPS (Fig. 1).

\section{Results and discussion}

\subsection{Retrosynthetic strategy}

The CPS of $S$. pneumoniae type 14 is built up from the tetrasaccharide repeating unit $\rightarrow 3)-\beta-\mathrm{D}-\mathrm{Gal} p-(1 \rightarrow 4)-\beta-$ D-Glc $p-(1 \rightarrow 6)-[\beta-D-G a l p-(1 \rightarrow 4)]-\beta-D-G l c p$ NAc- $(1 \rightarrow$ .${ }^{18}$ The title oligosaccharides (Fig. 1) represent either one (1) or two (2) tetrasaccharide-repeating units in which each repeating unit is built up from a linear backbone and a branched galactose residue. In a chemical approach to $\mathbf{1}$ and $\mathbf{2}$, first the intermediate linear trisaccharide 3 (Scheme 1) and hexasaccharide 4 (Scheme 2), respectively, were synthesized. Galactose residues were $\beta$ - $(1 \rightarrow 4)$-attached to the internal $N$ acetyl- $\beta$-D-glucosamine residues by using bovine milk $\beta$-1,4-galactosyltransferase (EC 2.4.1.22) and UDP-galactose.

\subsection{Synthesis of tetrasaccharide fragment 1}

In an earlier report, ${ }^{17}$ we have shown that by using the trisaccharide donor $(2,3,4,6$-tetra- $O$-acetyl- $\beta$-D-galactopyranosyl)-( $1 \rightarrow 4)$-(2,3,6-tri- $O$-acetyl- $\beta$-D-glucopyranosyl)-(1 $\rightarrow 6)$-2-deoxy-3,4-di- $O$ - $p$-methylbenzoyl-2-phthalimido- $\beta$-D-glucopyranosyl trichloroacetimidate (20) (Scheme 2) a linear tetra- and pentasaccharide could be synthesized. However, coupling of donor $\mathbf{2 0}$ with the spacer 6-azido-1-hexanol (6) could not be successfully realized. Therefore, an alternative route was followed to synthesize trisaccharide 3 (Scheme 1). To this end, two building blocks, 6-azidohexyl 2-deoxy-3,4-di-O- $P$ methylbenzoyl-2-phthalimido- $\beta$-D-glucopyranoside acceptor (11) and (2,3,4,6-tetra- $O$-acetyl- $\beta$-D-galactopyranosyl)-( $1 \rightarrow 4)-2,3,6$-tri- $O$-acetyl- $\beta$-D-glucopyranosyl
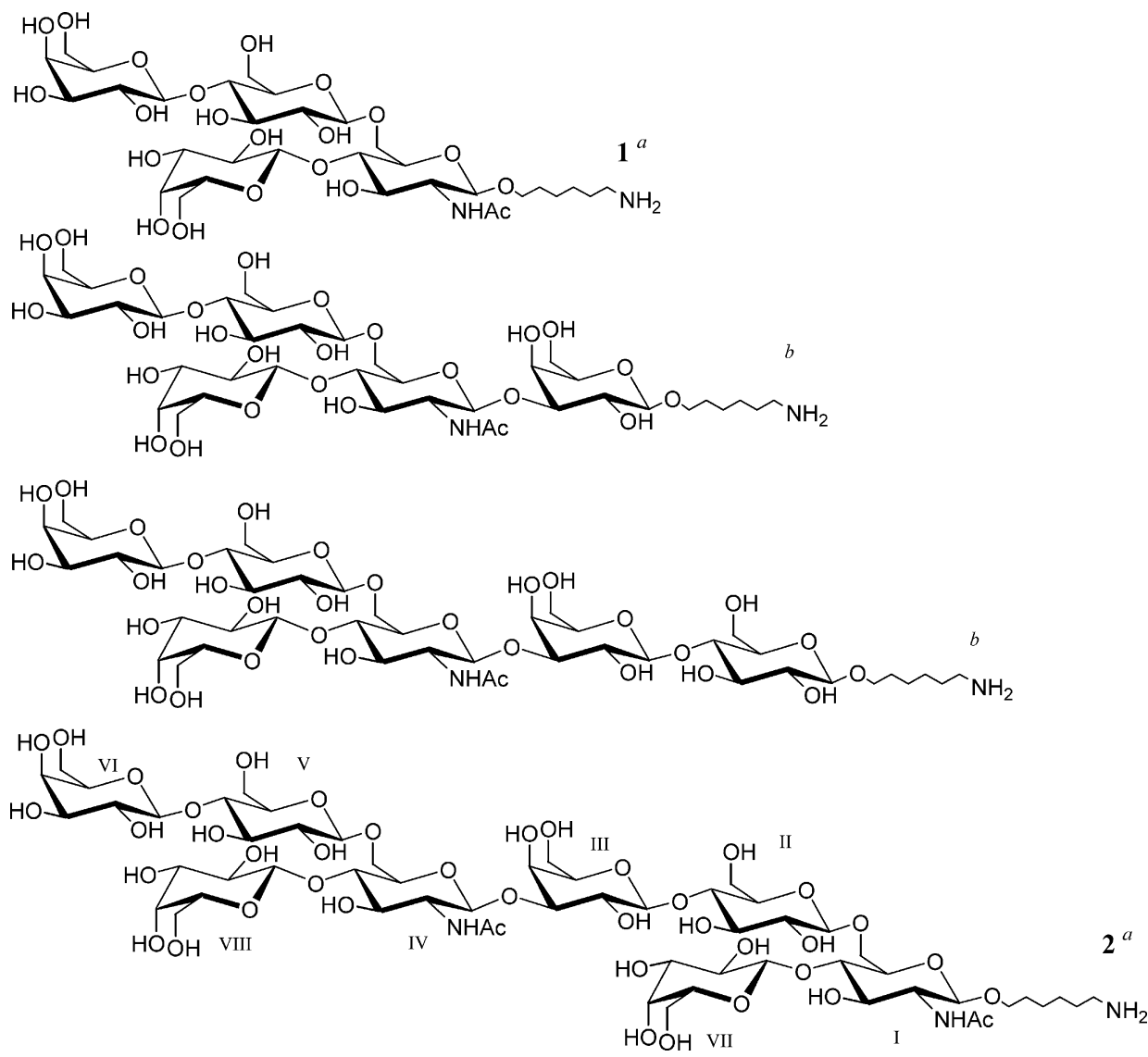

Fig. 1. Overview of 6-aminohexyl-spacered oligosaccharides, representing fragments varying in length between one and two repeating units of the capsular polysaccharide of $S$. pneumoniae type 14. (a) Synthesis described in this paper (compounds 1 and 2 ). (b) Synthesis described by Michalik and co-workers. ${ }^{17}$ 

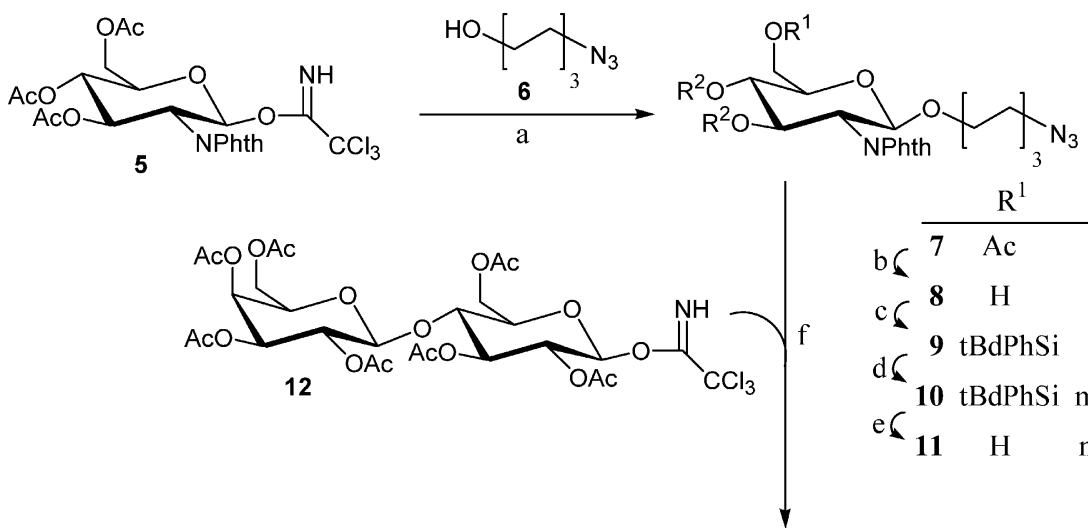

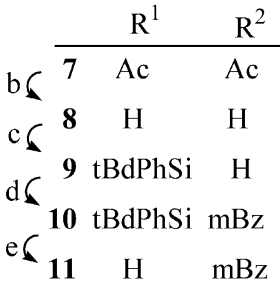

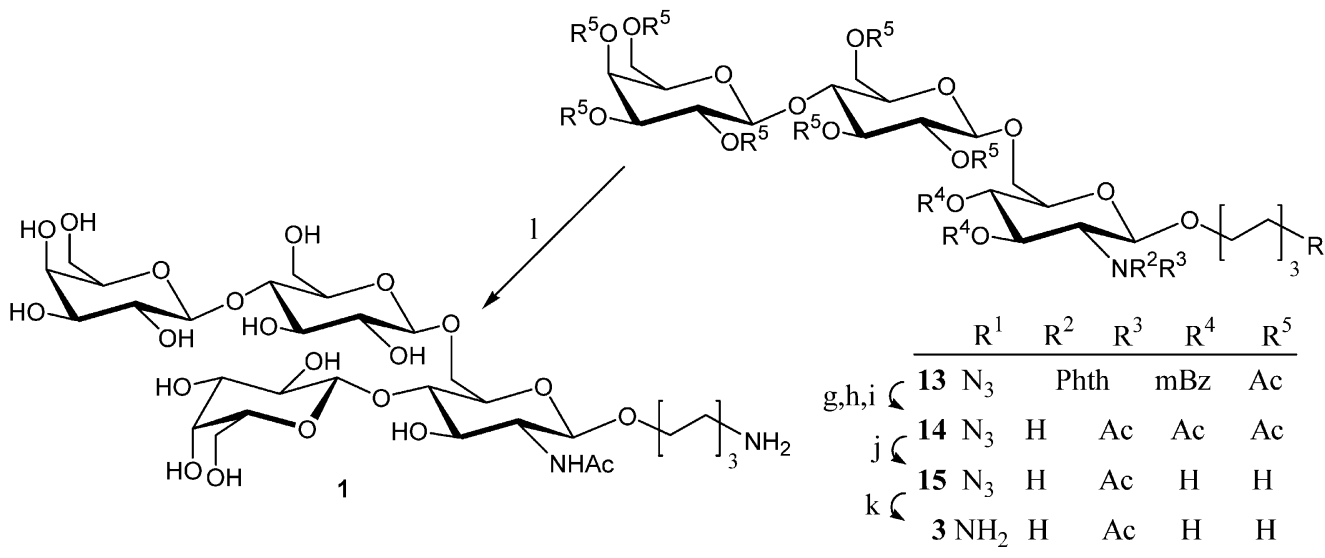

Scheme 1. Synthesis of tetrasaccharide 1: (a) 0.5 equiv $\mathrm{BF}_{3} \cdot \mathrm{Et}_{2} \mathrm{O}, 0{ }^{\circ} \mathrm{C}, \mathrm{CH}_{2} \mathrm{Cl}_{2}, 89 \%$; (b) $\mathrm{NaOMe}(\mathrm{pH} 8), \mathrm{MeOH} \mathrm{CH}_{2} \mathrm{Cl}_{2}$, quantitative; (c) $t$ - BdPhSiCl, DMAP, $\mathrm{Et}_{3} \mathrm{~N}$, pyridine, $\mathrm{CH}_{2} \mathrm{Cl}_{2}, 88 \%$; (d) $p$ - $\mathrm{mBzCl}, 0{ }^{\circ} \mathrm{C}$, pyridine, $\mathrm{CH}_{2} \mathrm{Cl}_{2}, 69^{\circ} \%$; (e) $\mathrm{AcCl}, 0{ }^{\circ} \mathrm{C}$, $\mathrm{MeOH}$, toluene, 75\%; (f) 10\% TMSOTf, $0{ }^{\circ} \mathrm{C}, \mathrm{CH}_{2} \mathrm{Cl}_{2}, 45 \%$; (g) NaOMe (pH 10), $\mathrm{MeOH}, \mathrm{CH}_{2} \mathrm{Cl}_{2} ;(\mathrm{h}) \mathrm{NH}_{2} \mathrm{CH}_{2} \mathrm{CH}_{2} \mathrm{NH}_{2}, 90{ }^{\circ} \mathrm{C}, 1-$ $\mathrm{BuOH}$; (i) pyridine, $\mathrm{Ac}_{2} \mathrm{O}, 86 \%$ over three steps; (j) $\mathrm{NaOMe}(\mathrm{pH} 10), \mathrm{MeOH}, \mathrm{CH}_{2} \mathrm{Cl}_{2}, 90 \%$; (k) 10\% $\mathrm{Pd}-\mathrm{C}, \mathrm{H}_{2}, \mathrm{aq} 25 \% \mathrm{NH}$, $t$ $\mathrm{BuOH}$, water, 71\%; (1) 1.8 equiv UDP-Gal, aq $50 \mathrm{mM}$ sodium cacodylate buffer (pH 7.5), $2.5 \mathrm{U} \beta-1$,4-galactosyltransferase, $12 \mathrm{U}$ alkaline phosphatase, $37^{\circ} \mathrm{C}, 91 \%$.

trichloroacetimidate donor $(\mathbf{1 2})^{19}$ were designed (Scheme 1). As a first step in the synthesis of 11, 3,4,6tri- $O$-acetyl-2-deoxy-2-phthalimido- $\beta$-D-glucopyranosyl trichloroacetimidate $(\mathbf{5})^{20}$ was coupled to 6-azido-1hexanol (6) using 0.5 equiv boron trifluoride ethyl etherate $\left(\mathrm{BF}_{3} \cdot \mathrm{Et}_{2} \mathrm{O}\right)$ as a catalyst at $0{ }^{\circ} \mathrm{C}$ in dry dichloromethane, giving 7 in $89 \%$ yield. After O-deacetylation of $\mathbf{7}$ using sodium methoxide at $\mathrm{pH} 8(\rightarrow \mathbf{8})$, a tert-butyldiphenylsilyl group $(t \mathrm{BdPhSi})$ was selectively introduced at the primary hydroxyl function of $\mathbf{8}$ using tert-butyldiphenylsilyl chloride in pyridine in the presence of a catalytic amount of 4-dimethylaminopyridine (DMAP), affording 9 in 88\% yield over two steps. The remaining free hydroxyl functions at O-3 and O-4 of 9 were protected with a toluoyl group $(\mathrm{mBz})$ by using $p$ methylbenzoyl chloride in pyridine at $0{ }^{\circ} \mathrm{C}(\rightarrow \mathbf{1 0}, 69 \%)$. The toluoyl group was chosen to avoid migration of an ester group from O-4 to O-6 after removal of the silyl group at O-6. ${ }^{21}$ Finally, removal of the tert-butyldiphenylsilyl ether group by using acetyl chloride in dry methanol yielded monosaccharide acceptor 11 (75\%). Coupling of 12 and 11 , at $0{ }^{\circ} \mathrm{C}$, using $10 \%$ trimethylsilyl trifluoromethanesulfonate (TMSOTf) as a catalyst gave trisaccharide 13 in a moderate yield (45\%). The main side-product was the 6-O-acetylated acceptor, which was isolated in $47 \%$ yield. This product, probably formed via the orthoester intermediate, could be converted back into acceptor $\mathbf{1 1}$ by treatment with acetyl chloride in dry methanol (data not shown). No attempts were made to improve the yield of this condensation reaction. O-Deacylation of 13 using sodium methoxide at $\mathrm{pH} 10$, followed by N-dephthaloylation by treatment with 1,2diaminoethane in 1-butanol at $90{ }^{\circ} \mathrm{C}$, and subsequent $\mathrm{N}, \mathrm{O}$-acetylation using acetic anhydride in pyridine afforded 14 in $86 \%$ yield over three steps. The Oacetylation step was carried out to facilitate chromatographic purification. O-Deacetylation of $\mathbf{1 4}$ using sodium methoxide at $\mathrm{pH} 10$ gave 15 (90\%). Finally, catalytic hydrogenation of the azido group of $\mathbf{1 5}$ using $10 \%$ palladium on charcoal and $\mathrm{H}_{2}$ in the presence of ammonia, yielded linear backbone 3 (71\%). Tetrasaccharide 1 was synthesized in $91 \%$ yield by transfer of galactose from UDP-galactose to O- 4 of the $N$-acetyl- $\beta$ D-glucosamine residue of 3 by using bovine milk $\beta-1,4-$ galactosyltransferase as a catalyst (Scheme 1). Alkaline phosphatase was added to the incubation mixture to 


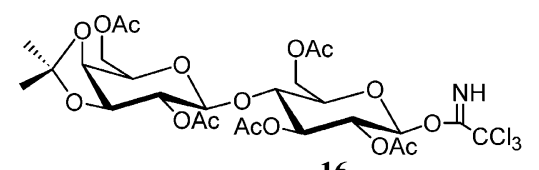

16
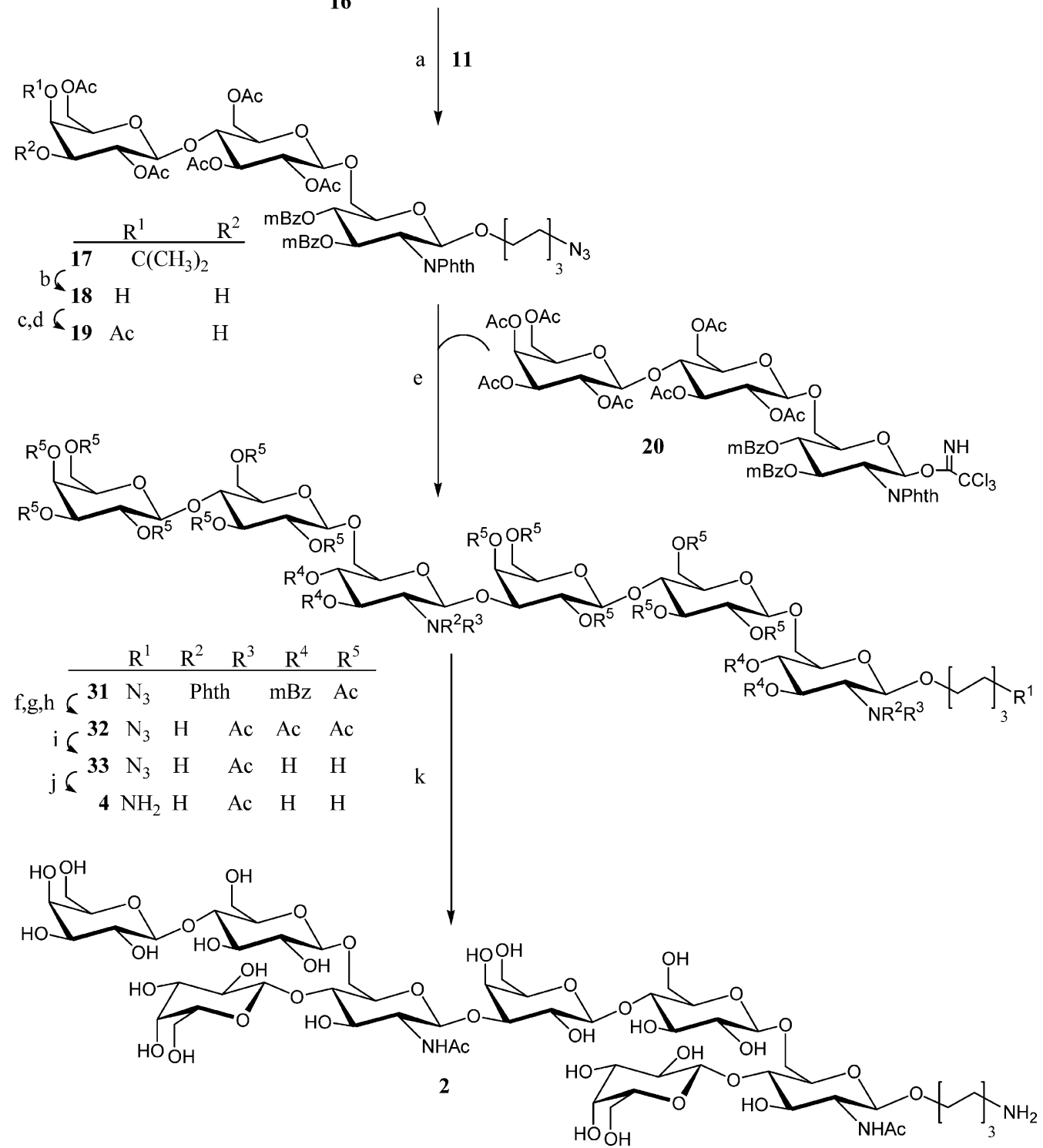

Scheme 2. Synthesis of octasaccharide 2: (a) $66 \%$ TMSOTf, $0{ }^{\circ} \mathrm{C}, \mathrm{CH}_{2} \mathrm{Cl}_{2}, 62 \%$; (b) TFA, $0{ }^{\circ} \mathrm{C}$, water, $\mathrm{CH}_{2} \mathrm{Cl}_{2}, 84 \%$; (c) trimethyl orthoacetate, $p$-TsOH, $\mathrm{CH}_{3} \mathrm{CN}$; (d) $\mathrm{AcOH}$, water, $85 \%$ over two steps; (e) $10 \%$ TMSOTf, $-20{ }^{\circ} \mathrm{C}, \mathrm{CH}_{2} \mathrm{Cl}_{2}, 12 \%$; (f) $\mathrm{NaOMe}$ (pH 10), $\mathrm{MeOH}, \mathrm{CH}_{2} \mathrm{Cl}_{2}$; (g) $\mathrm{NH}_{2} \mathrm{CH}_{2} \mathrm{CH}_{2} \mathrm{NH}_{2}, 80{ }^{\circ} \mathrm{C}, 1-\mathrm{BuOH}$; (h) pyridine, $\mathrm{Ac} 2 \mathrm{O}, 71 \%$ over three steps; (i) $\mathrm{NaOMe}$ (pH 10), $\mathrm{MeOH}$, $89 \%$; (j) $10 \% \mathrm{Pd}-\mathrm{C}, \mathrm{H}_{2}$, aq 25\% $\mathrm{NH}_{3}, t$ - $\mathrm{BuOH}$, water, 67\%; (k) 3 equiv UDP-Gal, aq $50 \mathrm{mM}$ sodium cacodylate buffer (pH 7.5), 1 $\mathrm{U} \beta$-1,4-galactosyltransferase, $10 \mathrm{U}$ alkaline phosphatase, $37{ }^{\circ} \mathrm{C}, 22 \%$.

prevent feedback inhibition by released UDP, thereby facilitating a high conversion of $\mathbf{3}$. Chemical and chemoenzymatic syntheses of structure 1, containing other functionalities at the anomeric center have been described and reviewed by Kamerling. ${ }^{22}{ }^{1} \mathrm{H}$ NMR data of 15,3 , and 1 are presented in Tables $1-3$, respectively.

\subsection{Synthesis of octasaccharide fragment 2}

Coupling of (2,6-di- $O$-acetyl-3,4-di- $O$-isopropylidene- $\beta$ D-galactopyranosyl)-( $1 \rightarrow 4)-2,3,6$-tri- $O$-acetyl- $\beta$-D-glucopyranosyl trichloroacetimidate donor $(\mathbf{1 6})^{23}$ to glucosamine acceptor 11 (Scheme 2) at $0{ }^{\circ} \mathrm{C}$, using $66 \%$ 
Table 1

$500 \mathrm{MHz}{ }^{1} \mathrm{H}$ NMR data (TOCSY, ROESY) of 15 at $300 \mathrm{~K}$ (in ppm)

\begin{tabular}{llll}
\hline Proton & \multicolumn{1}{l}{$\delta_{\mathrm{H}}$} & & \\
\cline { 2 - 4 } & GlcNAcI & GlcII & GalIII \\
\hline $\mathrm{H}-1$ & 4.51 & 4.55 & 4.45 \\
$\mathrm{H}-2$ & 3.68 & 3.38 & 3.53 \\
$\mathrm{H}-3$ & 3.52 & 3.67 & 3.66 \\
$\mathrm{H}-4$ & 3.52 & 3.67 & 3.93 \\
$\mathrm{H}-5$ & 3.62 & 3.61 & n.d. $^{\text {a }}$ \\
$\mathrm{H}-6 \mathrm{a}$ & 4.21 & 3.98 & n.d. \\
$\mathrm{H}-6 \mathrm{~b}$ & 3.89 & 3.82 & n.d. \\
\hline $\mathrm{O}\left(\mathrm{CH}_{2}\right)_{2}\left(\mathrm{CH}_{2}\right)_{2}\left(\mathrm{CH}_{2}\right)_{2} \mathrm{~N}_{3}$ & & & $1.34-1.37(4 \mathrm{H})$ \\
$\mathrm{OCH}$ & $\mathrm{CH}_{2}\left(\mathrm{CH}_{2}\right)_{2} \mathrm{CH}_{2} \mathrm{CH}_{2} \mathrm{~N}_{3}$ & & $1.54-1.61(4 \mathrm{H})$ \\
$\mathrm{CH} \mathrm{N}_{3}$ & & & 3.32 \\
$\mathrm{OCH}_{2}\left(\mathrm{CH}_{2}\right)_{5} \mathrm{~N}_{3}$ & & & $3.61,3.89$ \\
$\mathrm{NDCOCH}_{3}$ & & & 2.03 \\
\hline
\end{tabular}

a n.d., not determined.

trimethylsilyl trifluoromethanesulfonate, gave trisaccharide $17(62 \%)$. $O$-Deisopropylidenation of 17 with aqueous $90 \%$ trifluoroacetic acid $(\rightarrow \mathbf{1 8}, 84 \%$ ), followed by selective acetylation at $\mathrm{O}-4$ of the galactose residue via orthoester formation using trimethyl orthoacetate and $p$-toluenesulfonic acid, and subsequent ring opening with aqueous $80 \%$ acetic acid afforded $19(85 \%$ over two steps). ${ }^{24}$ A small amount of side-product $(6 \%)$ was formed in which O-3 of the galactose residue was acetylated, either due to acetyl migration or ring opening to O-3. Coupling of 19 with $(2,3,4,6$-tetra- $O$-acetyl$\beta$-D-galactopyranosyl)-( $1 \rightarrow 4)-(2,3,6$-tri- $O$-acetyl- $\beta$-Dglucopyranosyl)-(1 $\rightarrow 6$ )-2-deoxy-3,4-di- $O$ - $p$-methylbenzoyl-2-phthalimido- $\beta$-D-glucopyranosyl trichloroacetimidate $(\mathbf{2 0})^{17}$ in dichloromethane at $-20{ }^{\circ} \mathrm{C}$, using
$10 \%$ trimethylsilyl trifluoromethanesulfonate as a catalyst, gave hexasaccharide 31 in only $12 \%$ yield (Scheme 2 ). The low yield is probably due to the low reactivity of the free O-3 position, since the acceptor could be isolated after the reaction. Other coupling attempts, at different temperatures and with different catalysts, did not improve the yield of the reaction.

Therefore, in a second approach, an attempt was made to synthesize hexasaccharide $\mathbf{3 1}$ via a slightly different route in which acetyl ester groups were replaced by benzyl ether functions. O-Deacylation of $\mathbf{1 7}$, followed by benzylation with benzyl bromide in dry $N, N$-dimethylformamide in the presence of sodium hydride resulted also in the removal of the phthalimido protective group. Introduction of benzyl groups via

Table 2

$500 \mathrm{MHz}{ }^{1} \mathrm{H}$ NMR data (TOCSY, ROESY) of 3 at $300 \mathrm{~K}$ (in ppm)

\begin{tabular}{|c|c|c|c|}
\hline \multirow[t]{2}{*}{ Proton } & \multicolumn{3}{|l|}{$\delta_{\mathrm{H}}$} \\
\hline & GlcNAcI & GlcII & GalIII \\
\hline $\mathrm{H}-1$ & 4.51 & 4.55 & 4.45 \\
\hline $\mathrm{H}-2$ & 3.68 & 3.37 & 3.55 \\
\hline $\mathrm{H}-3$ & 3.53 & 3.67 & 3.67 \\
\hline $\mathrm{H}-4$ & 3.52 & 3.67 & 3.93 \\
\hline $\mathrm{H}-5$ & 3.62 & 3.62 & n.d. ${ }^{a}$ \\
\hline H-6a & 4.21 & 3.99 & n.d. \\
\hline $\mathrm{H}-6 \mathrm{~b}$ & 3.89 & 3.82 & n.d. \\
\hline $\mathrm{O}\left(\mathrm{CH}_{2}\right)_{2}\left(\mathrm{CH}_{2}\right)_{2}\left(\mathrm{CH}_{2}\right)_{2} \mathrm{ND}_{2}$ & & & $1.32-1.34(4 \mathrm{H})$ \\
\hline $\mathrm{OCH}_{2} \mathrm{CH}_{2}\left(\mathrm{CH}_{2}\right)_{2} \mathrm{CH}_{2} \mathrm{CH}_{2} \mathrm{ND}_{2}$ & & & $1.53-1.56(2 \mathrm{H}), 1.62-1.68(2 \mathrm{H})$ \\
\hline $\mathrm{CH}_{2} \mathrm{ND}_{2}$ & & & 2.98 \\
\hline $\mathrm{OCH}_{2}\left(\mathrm{CH}_{2}\right)_{5} \mathrm{ND}_{2}$ & & & $3.58,3.92$ \\
\hline $\mathrm{NDCOCH}_{3}$ & & & 2.03 \\
\hline
\end{tabular}

\footnotetext{
${ }^{a}$ n.d., not determined.
} 
Table 3

$500 \mathrm{MHz}{ }^{1} \mathrm{H}$ NMR data (TOCSY, ROESY) of 1 at $300 \mathrm{~K}$ (in ppm)

\begin{tabular}{|c|c|c|c|c|}
\hline \multirow[t]{2}{*}{ Proton } & \multicolumn{4}{|l|}{$\delta_{\mathrm{H}}$} \\
\hline & GlcNAcI & GlcII & GallII $^{a}$ & GalVII $^{b}$ \\
\hline H-1 & 4.54 & 4.56 & 4.45 & 4.53 \\
\hline $\mathrm{H}-2$ & 3.74 & 3.38 & 3.55 & 3.53 \\
\hline $\mathrm{H}-3$ & 3.69 & 3.67 & 3.68 & 3.68 \\
\hline $\mathrm{H}-4$ & 3.82 & 3.67 & 3.92 & 3.92 \\
\hline $\mathrm{H}-5$ & 3.72 & 3.62 & n.d. ${ }^{\mathrm{c}}$ & n.d. \\
\hline H-6a & 4.29 & 3.98 & 3.73 & 3.73 \\
\hline $\mathrm{H}-6 \mathrm{~b}$ & 3.96 & 3.82 & 3.78 & 3.78 \\
\hline $\mathrm{O}\left(\mathrm{CH}_{2}\right)_{2}\left(\mathrm{CH}_{2}\right)_{2}\left(\mathrm{CH}_{2}\right)_{2} \mathrm{ND}_{2}$ & & & $1.36-1.38(4 \mathrm{H})$ & \\
\hline $\mathrm{OCH}_{2} \mathrm{CH}_{2}\left(\mathrm{CH}_{2}\right)_{2} \mathrm{CH}_{2} \mathrm{CH}_{2} \mathrm{ND}_{2}$ & & & $1.55-1.58(2 \mathrm{H}), 1.62-1.67(2 \mathrm{H})$ & \\
\hline $\mathrm{CH}_{2} \mathrm{ND}_{2}$ & & & 2.98 & \\
\hline $\mathrm{OCH}_{2}\left(\mathrm{CH}_{2}\right)_{5} \mathrm{ND}_{2}$ & & & $3.60,3.92$ & \\
\hline $\mathrm{NDCOCH}_{3}$ & & & 2.03 & \\
\hline
\end{tabular}

a $\operatorname{Gal}(\beta 1-4) \mathrm{Glc}$.

b Gal $(\beta 1-4)$ GlcNAc.

c n.d., not determined.

coupling of the benzyl-protected lactose donor, with acceptor 11, yielded $\alpha / \beta$-mixtures, which could not be fractionated. In an alternative approach to obtain 31, trisaccharide donor $\mathbf{2 8}$ was designed to be combined with 11 and 12 (Scheme 3). Coupling of 3,4,6-tri- $O$ acetyl-2-deoxy-2-phthalimido- $\beta$-D-glucopyranosyl tri-
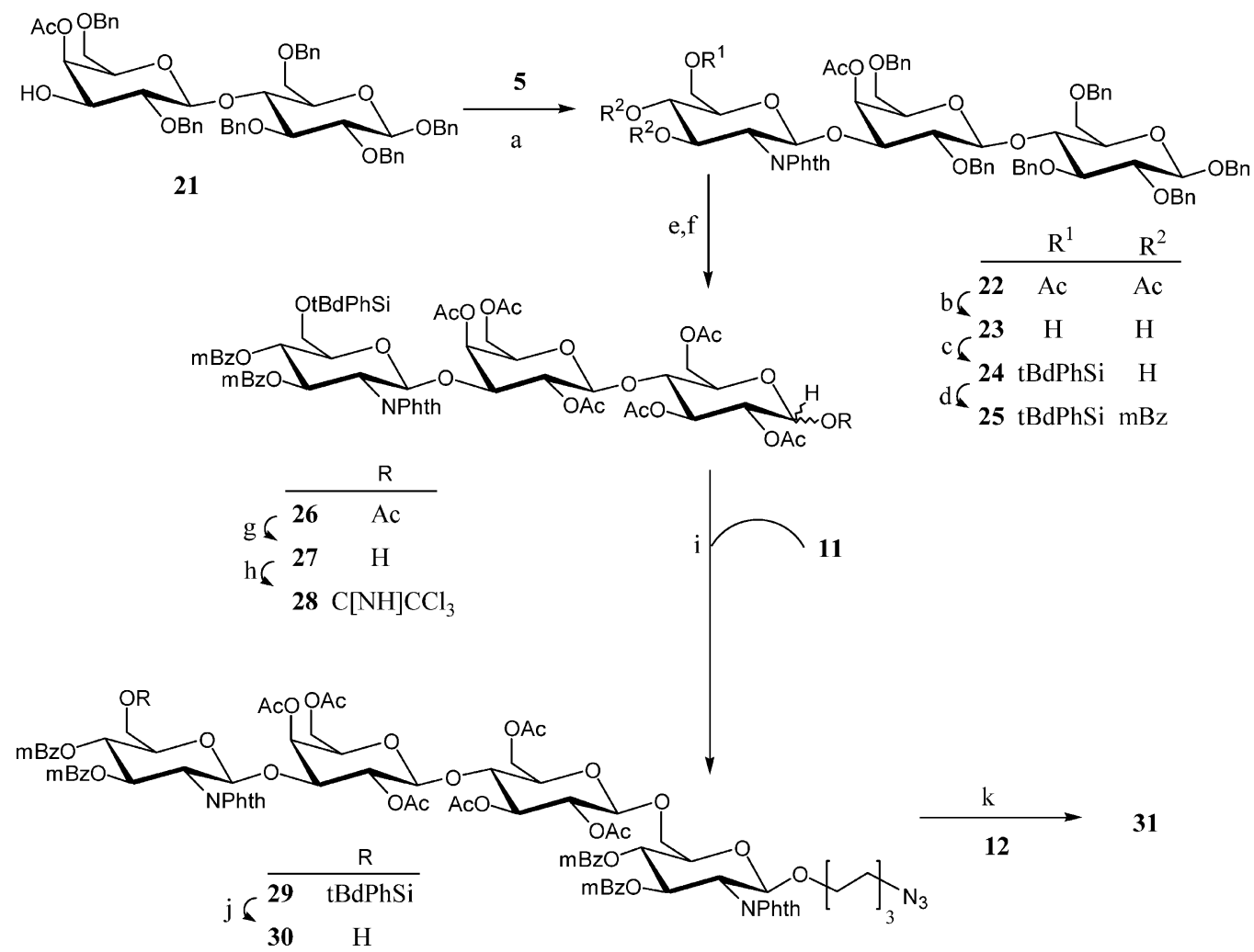

Scheme 3. Alternative synthesis of hexasaccharide backbone 31: (a) $8 \%$ TMSOTf, $-70{ }^{\circ} \mathrm{C}, \mathrm{CH}_{2} \mathrm{Cl}_{2}, 86 \%$; (b) $\mathrm{NaOMe}$ (pH 9), $\mathrm{MeOH}, \mathrm{CH}_{2} \mathrm{Cl}_{2}, 82 \%$; (c) $t$ - $\mathrm{BdPhSiCl}$, DMAP, $\mathrm{Et}_{3} \mathrm{~N}$, pyridine, $\mathrm{CH}_{2} \mathrm{Cl}_{2}, 89 \%$; (d) $p$-mBzCl, $0{ }^{\circ} \mathrm{C}$, pyridine, $\mathrm{CH}_{2} \mathrm{Cl}_{2}, 74 \%$; (e) $10 \%$ $\mathrm{Pd}-\mathrm{C}, \mathrm{H}_{2}, \mathrm{EtOH}, \mathrm{EtOAc}, 90 \%$; (f) pyridine, $\mathrm{Ac}_{2} \mathrm{O}$, quantitative; (g) hydrazinium acetate, DMF, $65 \%$; (h) $\mathrm{CCl}_{3} \mathrm{CN}, \mathrm{DBU}, 0{ }^{\circ} \mathrm{C}$, $\mathrm{CH}_{2} \mathrm{Cl}_{2}, 69 \%$; (i) $10 \%$ TMSOTf, $-40{ }^{\circ} \mathrm{C}, \mathrm{CH}_{2} \mathrm{Cl}_{2}, 38 \%$; (j) $1.0 \mathrm{M}$ TBAF in THF, $\mathrm{AcOH}, 0{ }^{\circ} \mathrm{C}, 90 \%$; (k) TMSOTf, $-40{ }^{\circ} \mathrm{C}$, $\mathrm{CH}_{2} \mathrm{Cl}_{2}, 60 \%$. 
chloroacetimidate $(\mathbf{5})^{20}$ to (4- $O$-acetyl-2,6-di- $O$-benzyl$\beta$-D-galactopyranosyl)-( $1 \rightarrow 4)-1,2,3,6$-tetra- $O$-benzyl- $\beta$ D-glucopyranose $(\mathbf{2 1})^{25}$ in dichloromethane at $-70{ }^{\circ} \mathrm{C}$, using $8 \%$ trimethylsilyl trifluoromethanesulfonate as a catalyst gave $22(86 \%)$. Mild O-deacetylation of 22, using sodium methoxide ( $\mathrm{pH} 9$ ), afforded $\mathbf{2 3}(82 \%)$, with retention of the acetyl group at galactose O-4. Selective tert-butyldiphenylsilylation of O-6 of the glucosamine residue of $\mathbf{2 3}$ using tert-butyldiphenylsilyl chloride and a catalytic amount of 4-dimethylaminopyridine in pyridine $(\rightarrow \mathbf{2 4}, 89 \%$ ), followed by toluoylation of $\mathrm{O}-3$ and O-4 using $p$-methylbenzoyl chloride in pyridine, gave $\mathbf{2 5}$ (74\%). O-Debenzylation of 25 using $10 \%$ palladium on charcoal and $\mathrm{H}_{2}$ and subsequent $\mathrm{O}$-acetylation using acetic anhydride in pyridine yielded $\mathbf{2 6}$ in $90 \%$ over two steps. Selective removal of the anomeric $O$-acetyl group by treatment with hydrazinium acetate in dry $N, N$ dimethylformamide ( $\rightarrow \mathbf{2 7}, 65 \%$ ), followed by imidation using trichloroacetonitrile in the presence of 1,8-diazabicyclo[5.4.0]undec-7-ene (DBU) gave donor 28 in an $\alpha: \beta$ ratio of $9: 1(69 \%)$. Coupling of $\mathbf{2 8}$ to glucosamine acceptor 11 (Scheme 3) at $-40{ }^{\circ} \mathrm{C}$ using $10 \%$ trimethylsilyl trifluoromethanesulfonate as a catalyst afforded tetrasaccharide 29 (38\%). O-De-tert-butyldiphenylsilylation of 29 in a 1:1 mixture of $1.0 \mathrm{M}$ tetrabutylammonium fluoride (TBAF) in tetrahydrofuran and acetic acid ( $\mathrm{pH}$ 6) yielded $30(90 \%)$. It should be noted that removal of the tert-butyldiphenylsilyl group by using acetyl chloride in dry methanol at $0{ }^{\circ} \mathrm{C}$ also lead to the removal of acetyl groups. Coupling of tetrasaccharide acceptor 30 with 3 equiv of $(2,3,4,6$-tetra- $O$-acetyl- $\beta$-Dgalactopyranosyl)-( $(1 \rightarrow 4)$-2,3,6-tri- $O$-acetyl- $\beta$-D-glucopyranosyl trichloroacetimidate (12), ${ }^{19}$ using $10 \%$ trimethylsilyl trifluoromethanesulfonate as a catalyst at $-40{ }^{\circ} \mathrm{C}$, gave hexasaccharide $31(60 \%)$. O-Deacylation of 31 with sodium methoxide in a methanol-dichloromethane mixture, followed by $\mathrm{N}$-dephthaloylation by treatment with 1,2-diaminoethane in 1-butanol at $80{ }^{\circ} \mathrm{C}$, and subsequent $\mathrm{N}, \mathrm{O}$-acetylation using acetic anhydride in pyridine gave 32 ( $71 \%$ over three steps). $O$-Deacetylation of $\mathbf{3 2}$ using sodium methoxide at $\mathrm{pH}$ $10(\rightarrow 33 ; 89 \%)$, and catalytic hydrogenation of the azido group using $10 \%$ palladium on charcoal and $\mathrm{H}_{2}$ gave 4 in $67 \%$ yield. Finally, octasaccharide 2 was synthesized in $22 \%$ yield by transfer of galactose from UDP-galactose to O- 4 of the two $N$-acetyl- $\beta$-D-glucosamine residues of 4 by using bovine milk $\beta-1,4-$ galactosyltransferase as a catalyst (Scheme 2). The low yield of the reaction was due to purification problems. ${ }^{1} \mathrm{H}$ NMR data of $\mathbf{3 3}, \mathbf{4}$, and $\mathbf{2}$ are presented in Tables 4-6, respectively.

Conjugation of the oligosaccharides $\mathbf{1}$ and $\mathbf{2}$ to $\mathrm{CRM}_{197}$ (cross reactive material) and immunological studies are in progress.

\section{Experimental}

\subsection{General methods}

All chemicals were of reagent grade, and were used without further purification. Reactions were monitored by TLC on Silica Gel $60 \mathrm{~F}_{254}$ (E. Merck); after examination under UV light, compounds were visualized by heating with $10 \%(\mathrm{v} / \mathrm{v})$ ethanolic $\mathrm{H}_{2} \mathrm{SO}_{4}$, orcinol (2 mg/mL) in $20 \%$ (v/v) methanolic $\mathrm{H}_{2} \mathrm{SO}_{4}$, or ninhydrin $(1.5 \mathrm{mg} / \mathrm{mL})$ in $1-\mathrm{BuOH}$-water-AcOH $(38: 1.75: 0.25)$. In the work-up procedures of reaction mixtures, organic solns were washed with appropriate amounts of the indicated aq solns, then dried $\left(\mathrm{MgSO}_{4}\right)$, and concentrated under diminished pressure at $40{ }^{\circ} \mathrm{C}$. Column chromatography was performed on Silica Gel 60 (E. Merck, $0.063-0.200 \mathrm{~mm}$ ). Optical rotations were measured with a Perkin-Elmer 241 polarimeter, using a 10 $\mathrm{cm}, 1 \mathrm{~mL}$ cell. ${ }^{1} \mathrm{H}$ NMR spectra were recorded at $300 \mathrm{~K}$ with a Bruker AC $300(300 \mathrm{MHz})$ or a Bruker AMX 500 $(500 \mathrm{MHz})$ spectrometer; the $\delta_{\mathrm{H}}$ values are given in ppm relative to the signal for internal $\mathrm{Me}_{4} \mathrm{Si}\left(\delta_{\mathrm{H}} 0, \mathrm{CDCl}_{3}\right.$ and $\left.\mathrm{CD}_{3} \mathrm{OD}\right)$ or internal acetone $\left(\delta_{\mathrm{H}} 2.225, \mathrm{D}_{2} \mathrm{O}\right) .{ }^{13} \mathrm{C}$ NMR spectra (APT, $75 \mathrm{MHz}$ ) were recorded at $300 \mathrm{~K}$ with a Bruker AC 300 spectrometer; $\delta_{\mathrm{C}}$ values are given in ppm relative to the signal of $\mathrm{CDCl}_{3}\left(\delta_{\mathrm{C}} 76.9, \mathrm{CDCl}_{3}\right)$ or for internal acetone $\left(\delta_{\mathrm{C}} 30.89, \mathrm{D}_{2} \mathrm{O}\right)$. Two-dimensional ${ }^{1} \mathrm{H}-{ }^{1} \mathrm{H}$ TOCSY (mixing times 7 and $100 \mathrm{~ms}$ ), ROESY (mixing time $300 \mathrm{~ms}$ ), and ${ }^{1} \mathrm{H}-{ }^{13} \mathrm{C}$ correlated HSQC NMR spectra $(500 \mathrm{MHz})$ were recorded at $300 \mathrm{~K}$ with a Bruker AMX 500 spectrometer. Exact masses were measured by nano electrospray time-of-flight mass spectrometry (positive-ion mode) using a Micromass LCToF mass spectrometer at a resolution of 5000 FWHM. Gold-coated capillaries were loaded with 1 $\mu \mathrm{L}$ of sample (conc $20 \mu \mathrm{M})$ dissolved in a $1: 1(\mathrm{v} / \mathrm{v})$ mixture of $\mathrm{MeCN}$-water with $0.1 \%$ formic acid. Pentafluorophenylalanine was added as internal standard. The capillary voltage was set at $1500 \mathrm{~V}$ and the cone voltage was set at $30 \mathrm{~V}$. Elemental analyses were carried out at the Department of Organic Chemistry of the University of Nijmegen (The Netherlands).

\subsection{6-Azidohexyl 3,4,6-tri- $O$-acetyl-2-deoxy-2- phthalimido- $\beta$-D-glucopyranose (7)}

A soln of 3,4,6-tri- $O$-acetyl-2-deoxy-2-phthalimido- $\beta$-Dglucopyranosyl trichloroacetimidate $(\mathbf{5})^{20}(2.45 \mathrm{~g}, 4.23$ $\mathrm{mmol})$ and 6-azido-1-hexanol (6) $(1.3 \mathrm{~g}, 9.08 \mathrm{mmol})$ in dry $\mathrm{CH}_{2} \mathrm{Cl}_{2}(50 \mathrm{~mL})$, containing molecular sieves $4 \AA$ (400 mg), was stirred for $2 \mathrm{~h}$ under Ar. After cooling to $0{ }^{\circ} \mathrm{C}, \mathrm{BF}_{3} \cdot \mathrm{Et}_{2} \mathrm{O}(0.28 \mathrm{~mL}, 2.19 \mathrm{mmol})$ was added and the mixture was stirred for $1.5 \mathrm{~h}$, after which a second portion of $\mathrm{BF}_{3} \cdot \mathrm{Et}_{2} \mathrm{O}(0.1 \mathrm{~mL}, 0.79 \mathrm{mmol})$ was added, and the stirring was continued for $1.5 \mathrm{~h}$. The mixture was neutralized with $\mathrm{Et}_{3} \mathrm{~N}$, washed with aq satd 
Table 4

$500 \mathrm{MHz}{ }^{1} \mathrm{H}$ NMR data (TOCSY, ROESY) of 33 at $300 \mathrm{~K}$ (in ppm)

\begin{tabular}{|c|c|c|c|c|c|c|}
\hline \multirow[t]{2}{*}{ Proton } & \multicolumn{6}{|l|}{$\delta_{\mathrm{H}}$} \\
\hline & GlcNAcI $^{a}$ & GlcII $^{b}$ & $\mathrm{GaIII}^{\mathrm{c}}$ & GlcNAcIV $^{\mathrm{d}}$ & $\mathrm{GlcV}^{\mathrm{e}}$ & GalVI $^{\mathrm{f}}$ \\
\hline $\mathrm{H}-1$ & 4.52 & 4.55 & 4.44 & 4.70 & 4.55 & 4.46 \\
\hline $\mathrm{H}-2$ & 3.68 & 3.37 & 3.59 & 3.76 & 3.37 & 3.57 \\
\hline $\mathrm{H}-3$ & 3.52 & 3.66 & 3.72 & 3.56 & 3.66 & 3.68 \\
\hline $\mathrm{H}-4$ & n.d. ${ }^{g}$ & n.d. & 4.16 & n.d. & n.d. & 3.92 \\
\hline H-5 & 3.60 & n.d. & n.d. & 3.61 & n.d. & n.d. \\
\hline H-6a & 4.21 & 3.98 & n.d. & 4.22 & 3.98 & n.d. \\
\hline $\mathrm{H}-6 \mathrm{~b}$ & 3.88 & 3.81 & n.d. & 3.89 & 3.81 & n.d. \\
\hline $\mathrm{O}\left(\mathrm{CH}_{2}\right)_{2}\left(\mathrm{CH}_{2}\right)_{2}\left(\mathrm{CH}_{2}\right)_{2} \mathrm{~N}_{3}$ & & & & $1.33-1.37(4 \mathrm{H})$ & & \\
\hline $\mathrm{OCH}_{2} \mathrm{CH}_{2}\left(\mathrm{CH}_{2}\right)_{2} \mathrm{CH}_{2} \mathrm{CH}_{2} \mathrm{~N}_{3}$ & & & & $1.53-1.60(4 \mathrm{H})$ & & \\
\hline $\mathrm{CH}_{2} \mathrm{~N}_{3}$ & & & & 3.32 & & \\
\hline $\mathrm{OCH}_{2}\left(\mathrm{CH}_{2}\right)_{5} \mathrm{~N}_{3}$ & & & & $3.61,3.89$ & & \\
\hline $\mathrm{NDCOCH}_{3}$ & & & & $2.03(2)$ & & \\
\hline
\end{tabular}

${ }^{\text {a }} \operatorname{GlcNAc}\left(\beta 1-\mathrm{O}\left(\mathrm{CH}_{2}\right)_{6} \mathrm{~N}_{3}\right)$.

b $\mathrm{Glc}(\beta 1-6) \mathrm{GlcNAc}\left(\beta 1-\mathrm{O}\left(\mathrm{CH}_{2}\right)_{6} \mathrm{~N}_{3}\right)$.

c $\operatorname{Gal}(\beta 1-4) \mathrm{Glc}(\beta 1-6) \operatorname{GlcNAc}\left(\beta 1-\mathrm{O}\left(\mathrm{CH}_{2}\right)_{6} \mathrm{~N}_{3}\right)$.

d GlcNAc $(\beta 1-3)$ Gal.

e $\operatorname{Glc}(\beta 1-6)$ GlcNAc $(\beta 1-3)$ Gal.

${ }^{f} \operatorname{Gal}(\beta 1-4) \operatorname{Glc}(\beta 1-6) \operatorname{GlcNAc}(\beta 1-3) \mathrm{Gal}$.

g n.d., not determined.

$\mathrm{NaHCO}_{3}$ and water, dried, filtered, and concentrated. Column chromatography $\left(9: 1 \mathrm{CH}_{2} \mathrm{Cl}_{2}-\mathrm{EtOAc}\right)$ of the residue gave 7 , isolated as a slightly yellow syrup $(2.10 \mathrm{~g}$, $89 \%) ; R_{f} 0.66\left(9: 1 \quad \mathrm{CH}_{2} \mathrm{Cl}_{2}-\mathrm{EtOAc}\right) ;[\alpha]_{\mathrm{D}}^{20}+19^{\circ}$ ( c 1 ,
$\left.\mathrm{CHCl}_{3}\right) ;{ }^{1} \mathrm{H} \mathrm{NMR}\left(300 \mathrm{MHz}, \mathrm{CDCl}_{3}\right): \delta 1.12-1.17(\mathrm{~m}$, $\left.4 \mathrm{H}, 2 \mathrm{CH}_{2}\right), 1.23-1.29\left(\mathrm{~m}, 2 \mathrm{H}, \mathrm{CH}_{2}\right), 1.43-1.45$ (m, 2 $\left.\mathrm{H}, \mathrm{CH}_{2}\right), 1.86,2.03$, and $2.11\left(3 \mathrm{~s}\right.$, each $\left.3 \mathrm{H}, 3 \mathrm{COCH}_{3}\right)$, $3.06\left(\mathrm{t}, 2 \mathrm{H}, \mathrm{CH}_{2} \mathrm{~N}_{3}\right), 3.44(\mathrm{~m}, 1 \mathrm{H}, \mathrm{OCHH}), 4.17(\mathrm{dd}, 1$

Table 5

$500 \mathrm{MHz}{ }^{1} \mathrm{H}$ NMR data (TOCSY, ROESY) of 4 at $300 \mathrm{~K}$ (in ppm)

\begin{tabular}{|c|c|c|c|c|c|c|}
\hline \multirow[t]{2}{*}{ Proton } & \multicolumn{6}{|l|}{$\delta_{\mathrm{H}}$} \\
\hline & GlcNAcI $^{\mathrm{a}}$ & $\mathrm{GlcII}^{\mathrm{b}}$ & GalIII $^{\mathrm{c}}$ & GlcNAcIV $^{\mathrm{d}}$ & $\mathrm{GlcV}^{\mathrm{e}}$ & GalVI $^{\mathrm{f}}$ \\
\hline $\mathrm{H}-1$ & 4.51 & 4.56 & 4.43 & 4.70 & 4.56 & 4.45 \\
\hline $\mathrm{H}-2$ & 3.68 & 3.37 & 3.60 & 3.76 & 3.37 & 3.55 \\
\hline $\mathrm{H}-3$ & 3.52 & 3.67 & 3.72 & 3.55 & 3.67 & 3.67 \\
\hline $\mathrm{H}-4$ & n.d. ${ }^{\mathrm{g}}$ & n.d. & 4.16 & n.d. & n.d. & 3.93 \\
\hline $\mathrm{H}-5$ & n.d. & n.d. & n.d. & n.d. & n.d. & n.d. \\
\hline $\mathrm{H}-6 \mathrm{a}$ & 4.21 & n.d. & n.d. & 4.22 & n.d. & n.d. \\
\hline $\mathrm{H}-6 \mathrm{~b}$ & 3.88 & n.d. & n.d. & 3.89 & n.d. & n.d. \\
\hline $\mathrm{O}\left(\mathrm{CH}_{2}\right)_{2}\left(\mathrm{CH}_{2}\right)_{2}\left(\mathrm{CH}_{2}\right)_{2} \mathrm{ND}_{2}$ & & & & $1.35-1.39(4 \mathrm{H})$ & & \\
\hline $\mathrm{OCH}_{2} \mathrm{CH}_{2}\left(\mathrm{CH}_{2}\right)_{2} \mathrm{CH}_{2} \mathrm{CH}_{2} \mathrm{ND}_{2}$ & & & & $1.55-1.57(2 \mathrm{H}), 1.64-1.66(2 \mathrm{H})$ & & \\
\hline $\mathrm{CH}_{2} \mathrm{ND}_{2}$ & & & & 2.99 & & \\
\hline $\mathrm{OCH}_{2}\left(\mathrm{CH}_{2}\right)_{5} \mathrm{ND}_{2}$ & & & & $3.59,3.91$ & & \\
\hline $\mathrm{NDCOCH}_{3}$ & & & & $2.03(2)$ & & \\
\hline
\end{tabular}

a $\operatorname{GlcNAc}\left(\beta 1-\mathrm{O}\left(\mathrm{CH}_{2}\right)_{6} \mathrm{NH}_{2}\right)$.

b $\mathrm{Glc}(\beta 1-6) \mathrm{GlcNAc}\left(\beta 1-\mathrm{O}\left(\mathrm{CH}_{2}\right)_{6} \mathrm{NH}_{2}\right)$.

${ }^{c} \mathrm{Gal}(\beta 1-4) \mathrm{Glc}(\beta 1-6) \mathrm{GlcNAc}\left(\beta 1-\mathrm{O}\left(\mathrm{CH}_{2}\right)_{6} \mathrm{NH}_{2}\right)$.

d GlcNAc( $\beta 1-3)$ Gal.

e $\operatorname{Glc}(\beta 1-6) \operatorname{GlcNAc}(\beta 1-3) \mathrm{Gal}$.

${ }^{\text {f }} \operatorname{Gal}(\beta 1-4) \operatorname{Glc}(\beta 1-6) \operatorname{GlcNAc}(\beta 1-3) \mathrm{Gal}$.

g n.d., not determined. 
Table 6

$500 \mathrm{MHz}{ }^{1} \mathrm{H}$ NMR data (TOCSY, ROESY) of 2 at $300 \mathrm{~K}$ (in ppm)

\begin{tabular}{|c|c|c|c|c|c|c|c|c|}
\hline \multirow[t]{2}{*}{ Proton } & \multicolumn{8}{|l|}{$\delta_{\mathrm{H}}$} \\
\hline & $\begin{array}{l}\text { GlcNAcI } \\
\text { a }\end{array}$ & GlcII $^{b}$ & GalIII $^{c}$ & GlcNAcIV $^{\mathrm{d}}$ & $\mathrm{GlcV}^{\mathrm{e}}$ & GalVI $^{\mathrm{f}}$ & GalVII $^{\mathrm{g}}$ & GalVIII $^{\mathrm{h}}$ \\
\hline $\mathrm{H}-1$ & 4.53 & 4.56 & 4.43 & 4.70 & 4.56 & 4.46 & 4.54 & 4.54 \\
\hline $\mathrm{H}-2$ & 3.72 & 3.37 & 3.59 & 3.81 & 3.37 & 3.55 & 3.54 & 3.54 \\
\hline $\mathrm{H}-3$ & n.d. ${ }^{\mathrm{i}}$ & 3.67 & 3.72 & n.d. & 3.67 & n.d. & 3.67 & 3.67 \\
\hline $\mathrm{H}-4$ & n.d. & n.d. & 4.16 & n.d. & n.d. & 3.94 & 3.93 & 3.93 \\
\hline $\mathrm{H}-5$ & n.d. & 3.62 & n.d. & 3.73 & 3.62 & n.d. & n.d. & n.d. \\
\hline H-6a & 4.29 & 3.99 & n.d. & 4.28 & 3.99 & n.d. & n.d. & n.d. \\
\hline $\mathrm{H}-6 \mathrm{~b}$ & 3.95 & 3.83 & n.d. & 3.97 & 3.83 & n.d. & n.d. & n.d. \\
\hline $\mathrm{O}\left(\mathrm{CH}_{2}\right)_{2}\left(\mathrm{CH}_{2}\right)_{2}\left(\mathrm{CH}_{2}\right)_{2} \mathrm{ND}_{2}$ & & & & $1.38-1.42(4 \mathrm{H})$ & & & & \\
\hline $\mathrm{OCH}_{2} \mathrm{CH}_{2}\left(\mathrm{CH}_{2}\right)_{2} \mathrm{CH}_{2} \mathrm{CH}_{2} \mathrm{ND}_{2}$ & & & & $\begin{array}{l}1.54-1.57(2 \mathrm{H}), 1.63-1.66(2 \\
\mathrm{H})\end{array}$ & & & & \\
\hline $\mathrm{CH}_{2} \mathrm{ND}_{2}$ & & & & 2.98 & & & & \\
\hline $\mathrm{OCH}_{2}\left(\mathrm{CH}_{2}\right)_{5} \mathrm{ND}_{2}$ & & & & $3.68,3.92$ & & & & \\
\hline $\mathrm{NDCOCH}_{3}$ & & & & $2.03(2)$ & & & & \\
\hline
\end{tabular}

${ }^{\text {a }} \mathrm{GlcNAc}\left(\beta 1-\mathrm{O}\left(\mathrm{CH}_{2}\right)_{6} \mathrm{NH}_{2}\right)$.

${ }^{\text {b }} \mathrm{Glc}(\beta 1-6) \mathrm{GlcNAc}\left(\beta 1-\mathrm{O}\left(\mathrm{CH}_{2}\right)_{6} \mathrm{NH}_{2}\right)$.

${ }^{c} \mathrm{Gal}(\beta 1-4) \mathrm{Glc}(\beta 1-6) \operatorname{GlcNAc}\left(\beta 1-\mathrm{O}\left(\mathrm{CH}_{2}\right)_{6} \mathrm{NH}_{2}\right)$.

d GlcNAc( $\beta 1-3)$ Gal.

e $\operatorname{Glc}(\beta 1-6) \operatorname{GlcNAc}(\beta 1-3) \mathrm{Gal}$.

${ }^{f} \operatorname{Gal}(\beta 1-4) \operatorname{Glc}(\beta 1-6) \operatorname{GlcNAc}(\beta 1-3) \mathrm{Gal}$.

${ }^{g} \mathrm{Gal}(\beta 1-4) \mathrm{GlcNAc}\left(\beta 1-\mathrm{O}\left(\mathrm{CH}_{2}\right)_{6} \mathrm{NH}_{2}\right)$.

h $\mathrm{Gal}(\beta 1-4) \mathrm{GlcNAc}(\beta 1-3) \mathrm{Gal}$.

i n.d., not determined.

H, $\left.J_{5,6 \mathrm{a}} 2.3, J_{6 \mathrm{a}, 6 \mathrm{~b}} 12.3 \mathrm{~Hz}, \mathrm{H}-6 \mathrm{a}\right), 4.31$ (dd, $1 \mathrm{H}, J_{1,2} 8.5$, $\left.J_{2,3} 10.8 \mathrm{~Hz}, \mathrm{H}-2\right), 4.33$ (dd, $\left.1 \mathrm{H}, J_{5,6 \mathrm{~b}} 4.7 \mathrm{~Hz}, \mathrm{H}-6 \mathrm{~b}\right)$, 5.17 (dd, $\left.1 \mathrm{H}, J_{3,4} 9.1, J_{4,5} 9.8 \mathrm{~Hz}, \mathrm{H}-4\right), 5.36$ (d, $1 \mathrm{H}, \mathrm{H}-$ 1), 5.79 (dd, $1 \mathrm{H}, \mathrm{H}-3), 7.75-7.89$ (2 m, $4 \mathrm{H}, \mathrm{Phth}) ;{ }^{13} \mathrm{C}$ NMR (75.5 MHz, $\left.\mathrm{CDCl}_{3}\right): \delta$ 20.2, 20.4, and 20.5 $\left(\mathrm{COCH}_{3}\right), 25.1,26.0,28.4$, and $28.8\left(4 \mathrm{CH}_{2}\right), 50.9$ $\left(\mathrm{CH}_{2} \mathrm{~N}_{3}\right), 54.5(\mathrm{C}-2), 61.9$ and $69.6\left(\mathrm{C}-6, \mathrm{OCH}_{2}\right), 68.9$, 70.6, and 71.6 (C-3, C-4, C-5), 98.0 (C-1), 123.3, 131.1, and 134.2 (Phth), 169.3, 169.9, and $170.5\left(\mathrm{COCH}_{3}\right)$; HRMS of $\mathrm{C}_{26} \mathrm{H}_{32} \mathrm{~N}_{4} \mathrm{O}_{10}(\mathrm{M}, 560.211)$ : $\left[\mathrm{M}+\mathrm{NH}_{4}\right]^{+}$ found 578.250, calcd 578.254.

\subsection{6-Azidohexyl 2-deoxy-2-phthalimido- $\beta$-D- glucopyranoside (8)}

To a soln of 7 (2.60 g, $4.64 \mathrm{mmol})$ in $\mathrm{MeOH}(20 \mathrm{~mL})$ and $\mathrm{CH}_{2} \mathrm{Cl}_{2}(5 \mathrm{~mL})$ was added $\mathrm{NaOMe}(\mathrm{pH} 8)$. The mixture was stirred for $5 \mathrm{~h}$, then neutralized with Dowex $50 \times 8$ $\left(\mathrm{H}^{+}\right)$, filtered, and concentrated giving 8 as a white solid (2.01 g, quantitative); $R_{f} 0.42\left(4: 1 \mathrm{CH}_{2} \mathrm{Cl}_{2}-\mathrm{MeOH}\right)$; $[\alpha]_{\mathrm{D}}^{20}-15^{\circ}\left(c 1, \mathrm{CHCl}_{3}\right) ;{ }^{1} \mathrm{H} \mathrm{NMR}\left(300 \mathrm{MHz}, \mathrm{CD}_{3} \mathrm{OD}\right)$; $\delta 1.08-1.10\left(\mathrm{~m}, 4 \mathrm{H}, 2 \mathrm{CH}_{2}\right), 1.14-1.20\left(\mathrm{~m}, 2 \mathrm{H}, \mathrm{CH}_{2}\right)$, 1.38-1.42 (m, $\left.2 \mathrm{H}, \mathrm{CH}_{2}\right), 3.01\left(\mathrm{t}, 2 \mathrm{H}, \mathrm{CH}_{2} \mathrm{~N}_{3}\right), 3.73(\mathrm{dd}$, $\left.1 \mathrm{H}, J_{5,6 \mathrm{~b}} 4.9, J_{6 \mathrm{a}, 6 \mathrm{~b}} 11.8 \mathrm{~Hz}, \mathrm{H}-6 \mathrm{~b}\right), 3.84(\mathrm{~m}, 1 \mathrm{H}$, OCH $H$ ), 3.92 (dd, $\left.1 \mathrm{H}, J_{5,6 \mathrm{a}} 1.5 \mathrm{~Hz}, \mathrm{H}-6 \mathrm{a}\right), 3.96$ (dd, 1 $\left.\mathrm{H}, J_{1,2} 8.5, J_{2,3} 10.6 \mathrm{~Hz}, \mathrm{H}-2\right), 4.25\left(\mathrm{dd}, 1 \mathrm{H}, J_{3,4} 7.7 \mathrm{~Hz}\right.$,
H-3), 5.13 (d, $1 \mathrm{H}, \mathrm{H}-1), 7.81-7.90$ (m, $4 \mathrm{H}, \mathrm{Phth}) ;{ }^{13} \mathrm{C}$ NMR (75.5 MHz, CD 3 OD): $\delta$ 26.2, 26.9, 29.3, and 29.8 $\left(4 \mathrm{CH}_{2}\right), 51.8\left(\mathrm{CH}_{2} \mathrm{~N}_{3}\right), 58.2(\mathrm{C}-2), 62.4$ and $70.0(\mathrm{C}-6$, $\left.\mathrm{OCH}_{2}\right), 72.2,72.3$, and 77.9 (C-3, C-4, C-5), 99.3 (C-1), 123.8, 132.6, and 135.3 (Phth); HRMS of $\mathrm{C}_{20} \mathrm{H}_{26} \mathrm{~N}_{4} \mathrm{O}_{7}$ $(\mathrm{M}, 434.180):\left[\mathrm{M}+\mathrm{NH}_{4}\right]^{+}$found 452.212, calcd 452.214

\subsection{6-Azidohexyl 6-O-tert-butyldiphenylsilyl-2-deoxy-2- phthalimido- $\beta$-D-glucopyranoside (9)}

To a soln of $8(2.01 \mathrm{~g}, 4.64 \mathrm{mmol})$ in $\mathrm{CH}_{2} \mathrm{Cl}_{2}(30 \mathrm{~mL})$ were added Py $(3.0 \mathrm{~mL})$, a catalytic amount of 4dimethylaminopyridine, $\mathrm{Et}_{3} \mathrm{~N}(0.86 \mathrm{~mL})$, and tert-butyldiphenylsilylchloride $(1.7 \mathrm{~mL}, 6.54 \mathrm{mmol})$. The mixture was stirred for $16 \mathrm{~h}$, then poured into cold water, washed with aq satd $\mathrm{NaHCO}_{3}$, dried, filtered, and concentrated. Column chromatography (1:1 tolueneEtOAc) of the residue gave 9, isolated as a white foam [3.12 g, 88\% (starting from 7)]; $R_{f} 0.53$ (1:1 tolueneEtOAc); $[\alpha]_{\mathrm{D}}^{20}-16^{\circ}\left(c 1, \mathrm{CHCl}_{3}\right) ;{ }^{1} \mathrm{H} \mathrm{NMR}(300 \mathrm{MHz}$, $\left.\mathrm{CDCl}_{3}\right): \delta 1.06\left[\mathrm{~s}, 9 \mathrm{H},\left(\mathrm{CH}_{3}\right)_{3} \mathrm{CSi}\right], 1.06-1.13(\mathrm{~m}, 4 \mathrm{H}, 2$ $\left.\mathrm{CH}_{2}\right), 1.25-1.27\left(\mathrm{~m}, 2 \mathrm{H}, \mathrm{CH}_{2}\right), 1.39-1.41(\mathrm{~m}, 2 \mathrm{H}$, $\left.\mathrm{CH}_{2}\right), 3.02\left(\mathrm{t}, 2 \mathrm{H}, \mathrm{CH}_{2} \mathrm{~N}_{3}\right), 3.38(\mathrm{~m}, 1 \mathrm{H}, \mathrm{OCH}), 3.58$ (m, $1 \mathrm{H}, \mathrm{H}-5), 3.67$ (t, $1 \mathrm{H}, \mathrm{H}-4), 3.76$ (m, $1 \mathrm{H}, \mathrm{OCH} H)$, 3.96 (d, 2 H, $J_{5,6} 4.9$ Hz, H-6a, H-6b), 4.09 (dd, 1 H, $J_{1,2}$ 
8.4, $\left.J_{2,3} 10.9 \mathrm{~Hz}, \mathrm{H}-2\right), 4.36$ (dd, $1 \mathrm{H}, J_{3,4} 8.4 \mathrm{~Hz}, \mathrm{H}-3$ ), 5.17 (d, $1 \mathrm{H}, \mathrm{H}-1) ;{ }^{13} \mathrm{C}$ NMR (75.5 MHz, $\left.\mathrm{CDCl}_{3}\right): \delta 19.1$ [( $\left.\left(\mathrm{CH}_{3}\right)_{3} \mathrm{CSi}\right], 25.3,26.0,28.4$, and $29.0\left(4 \mathrm{CH}_{2}\right), 26.7$ [ $\left.\left(\mathrm{CH}_{3}\right)_{3} \mathrm{CSi}\right], 51.0\left(\mathrm{CH}_{2} \mathrm{~N}_{3}\right), 56.3(\mathrm{C}-2), 64.8$ and $69.1(\mathrm{C}-$ 6, $\left.\mathrm{OCH}_{2}\right), 71.7,74.1$, and 74.4 (C-3, C-4, C-5), 98.0 (C1); HRMS of $\mathrm{C}_{36} \mathrm{H}_{44} \mathrm{~N}_{4} \mathrm{O}_{7} \mathrm{Si}(\mathrm{M}, 672.297)$ : $\left[\mathrm{M}+\mathrm{NH}_{4}\right]^{+}$ found 690.332 , calcd 690.330 .

\subsection{6-Azidohexyl 6-O-tert-butyldiphenylsilyl-2-deoxy- 3,4-di- $O$ - $p$-methylbenzoyl-2-phthalimido- $\beta$-D- glucopyranoside (10)}

To a soln of $9(2.75 \mathrm{~g}, 4.09 \mathrm{mmol})$ in Py $(35 \mathrm{~mL})$ was added, at $0{ }^{\circ} \mathrm{C}$, dropwise a soln of $p$-methylbenzoyl chloride $(1.35 \mathrm{~mL}, 10.2 \mathrm{mmol})$ in dry $\mathrm{CH}_{2} \mathrm{Cl}_{2}(10 \mathrm{~mL})$. The mixture was stirred for $20 \mathrm{~h}$, then, washed with cold water and aq satd $\mathrm{NaHCO}_{3}$, dried, filtered, and concentrated. Column chromatography (19:1 tolueneEtOAc) of the residue gave 10, isolated as a colourless syrup $(3.25 \mathrm{~g}, 69 \%) ; R_{f} 0.65\left(7: 1\right.$ toluene-EtOAc); $[\alpha]_{\mathrm{D}}^{20}$ $-6^{\circ}\left(\mathrm{c} 1, \mathrm{CHCl}_{3}\right) ;{ }^{1} \mathrm{H}$ NMR $\left(300 \mathrm{MHz}, \mathrm{CDCl}_{3}\right): \delta 1.03$ [s, $9 \mathrm{H},\left(\mathrm{CH}_{3}\right)_{3} \mathrm{CSi}$ ], 1.16-1.19 (m, $\left.4 \mathrm{H}, 2 \mathrm{CH}_{2}\right), 1.25-$ $1.29\left(\mathrm{~m}, 2 \mathrm{H}, \mathrm{CH}_{2}\right), 1.49-1.55\left(\mathrm{~m}, 2 \mathrm{H}, \mathrm{CH}_{2}\right), 2.27$ and $2.35\left(2 \mathrm{~s}\right.$, each $\left.3 \mathrm{H}, 2 \mathrm{CH}_{3} \mathrm{C}_{6} \mathrm{H}_{4} \mathrm{CO}\right), 3.05(\mathrm{t}, 2 \mathrm{H}$, $\left.\mathrm{CH}_{2} \mathrm{~N}_{3}\right), 3.48(\mathrm{~m}, 1 \mathrm{H}, \mathrm{OCH} \mathrm{H}), 4.51\left(\mathrm{dd}, 1 \mathrm{H}, J_{1,2} 8.5\right.$, $\left.J_{2,3} 10.8 \mathrm{~Hz}, \mathrm{H}-2\right), 5.48$ (d, $\left.1 \mathrm{H}, \mathrm{H}-1\right), 5.57$ (t, $\left.1 \mathrm{H}, \mathrm{H}-4\right)$, $6.17\left(\mathrm{dd}, 1 \mathrm{H}, J_{3,4} 9.3 \mathrm{~Hz}, \mathrm{H}-3\right), 7.04$ and $7.13(2 \mathrm{~d}$, each $2 \mathrm{H}$, Phth); ${ }^{13} \mathrm{C}$ NMR $\left(75.5 \mathrm{MHz}, \mathrm{CDCl}_{3}\right): \delta 19.1$ $\left[\left(\mathrm{CH}_{3}\right)_{3} \mathrm{CSi}\right], 21.5\left(2 \mathrm{CH}_{3} \mathrm{C}_{6} \mathrm{H}_{4} \mathrm{CO}\right), 25.4,26.2,28.5$, and $29.1\left(4 \mathrm{CH}_{2}\right), 26.6\left[\left(\mathrm{CH}_{3}\right)_{3} \mathrm{CSi}\right], 51.1\left(\mathrm{CH}_{2} \mathrm{~N}_{3}\right), 55.0(\mathrm{C}-$ 2), 62.9 and $69.3\left(\mathrm{C}-6, \mathrm{OCH}_{2}\right), 69.7,71.3$, and $75.1(\mathrm{C}-3$, C-4, C-5), 98.0 (C-1), 164.9 and $165.0\left(2 \mathrm{CH}_{3} \mathrm{C}_{6} \mathrm{H}_{4} \mathrm{CO}\right)$; Anal. Calcd for $\mathrm{C}_{52} \mathrm{H}_{56} \mathrm{~N}_{4} \mathrm{O}_{9} \mathrm{Si}: \mathrm{C}, 68.70 ; \mathrm{H}, 6.21 ; \mathrm{N}$, 6.16. Found: C, 68.58; H, 6.26; N, 6.02 .

\subsection{6-Azidohexyl 2-deoxy-3,4-di- $O$-p-methylbenzoyl-2- phthalimido- $\beta$-D-glucopyranoside (11)}

To a soln of $\mathbf{1 0}(0.72 \mathrm{~g}, 0.79 \mathrm{mmol})$ in dry toluene $(5.0$ $\mathrm{mL})$ was added, at $0{ }^{\circ} \mathrm{C}$, a soln of acetyl chloride $(0.93$ $\mathrm{mL}, 13.05 \mathrm{mmol})$ in dry $\mathrm{MeOH}(25 \mathrm{~mL})$. The mixture was stirred under $\mathrm{Ar}$ for $4 \mathrm{~h}$, then neutralized with $\mathrm{Et}_{3} \mathrm{~N}$, washed with water, dried, filtered, and concentrated. Low-pressure column chromatography (5:1 tolueneEtOAc) of the residue gave 11, isolated as a colourless syrup $(0.40 \mathrm{~g}, 75 \%) ; R_{f} 0.28\left(4: 1\right.$ toluene-EtOAc); $[\alpha]_{\mathrm{D}}^{20}$ $-3^{\circ}\left(c \quad 1, \mathrm{CHCl}_{3}\right) ;{ }^{1} \mathrm{H}$ NMR $\left(300 \mathrm{MHz}, \mathrm{CDCl}_{3}\right): \delta$ $1.15-1.13\left(\mathrm{~m}, 4 \mathrm{H}, 2 \mathrm{CH}_{2}\right), 1.24-1.28\left(\mathrm{~m}, 2 \mathrm{H}, \mathrm{CH}_{2}\right)$, $1.43-1.49\left(\mathrm{~m}, 2 \mathrm{H}, \mathrm{CH}_{2}\right), 2.23$ and $2.30(2 \mathrm{~s}$, each $3 \mathrm{H}, 2$ $\left.\mathrm{CH}_{3} \mathrm{C}_{6} \mathrm{H}_{4} \mathrm{CO}\right), 3.04\left(\mathrm{t}, 2 \mathrm{H}, \mathrm{CH}_{2} \mathrm{~N}_{3}\right), 3.51(\mathrm{~m}, 1 \mathrm{H}$, $\mathrm{OC} H \mathrm{H}), 3.75\left(\mathrm{dd}, 1 \mathrm{H}, J_{5,6 \mathrm{~b}} 4.0, J_{6 \mathrm{a}, 6 \mathrm{~b}} 12.7 \mathrm{~Hz}, \mathrm{H}-6 \mathrm{~b}\right)$, $4.55\left(\mathrm{dd}, 1 \mathrm{H}, J_{1,2} 8.5, J_{2,3} 10.7 \mathrm{~Hz}, \mathrm{H}-2\right), 5.50(\mathrm{t}, 1 \mathrm{H}, \mathrm{H}-$ 4), 5.55 (d, $1 \mathrm{H}, \mathrm{H}-1), 6.31$ (dd, $1 \mathrm{H}, J_{3,4} 9.3 \mathrm{~Hz}, \mathrm{H}-3$ ), 7.02 and $7.13(2 \mathrm{~d}$, each $2 \mathrm{H}$, Phth), 7.66 and $7.83(2 \mathrm{~d}$, each $\left.4 \mathrm{H}, 2 \mathrm{CH}_{3} \mathrm{C}_{6} \mathrm{H}_{4} \mathrm{CO}\right) ;{ }^{13} \mathrm{C}$ NMR $(75.5 \mathrm{MHz}$, $\left.\mathrm{CDCl}_{3}\right): \delta 21.3$ and $21.4\left(2 \mathrm{CH}_{3} \mathrm{C}_{6} \mathrm{H}_{4} \mathrm{CO}\right), 25.2,26.0$,
28.4, and $28.9\left(4 \mathrm{CH}_{2}\right), 50.9\left(\mathrm{CH}_{2} \mathrm{~N}_{3}\right), 54.7(\mathrm{C}-2), 61.2$ and $69.5\left(\mathrm{C}-6, \mathrm{OCH}_{2}\right), 69.8,70.5$, and $74.3(\mathrm{C}-3, \mathrm{C}-4, \mathrm{C}-$ 5), 98.1 (C-1), 165.4 and $165.9\left(2 \mathrm{CH}_{3} \mathrm{C}_{6} \mathrm{H}_{4} \mathrm{CO}\right)$; HRMS of $\mathrm{C}_{36} \mathrm{H}_{38} \mathrm{~N}_{4} \mathrm{O}_{9} \quad(\mathrm{M}, 670.263):\left[\mathrm{M}+\mathrm{NH}_{4}\right]^{+}$found 688.294, calcd 688.298.

\subsection{6-Azidohexyl $(2,3,4,6$-tetra- $O$-acetyl- $\beta$-D- galactopyranosyl)-( $(1 \rightarrow 4)-(2,3,6$-tri- $O$-acetyl- $\beta$-D- glucopyranosyl)-(1 $\rightarrow 6)-2$-deoxy-3,4-di- $O$ - $p$ - methylbenzoyl-2-phthalimido- $\beta$-D-glucopyranoside (13)}

A soln of $(2,3,4,6$-tetra- $O$-acetyl- $\beta$-D-galactopyranosyl)$(1 \rightarrow 4)-2,3,6$-tri- $O$-acetyl- $\beta$-D-glucopyranosyl trichloroacetimidate $(\mathbf{1 2})^{19}(0.26 \mathrm{~g}, 0.33 \mathrm{mmol})$ and $11(0.17 \mathrm{~g}$, $0.26 \mathrm{mmol})$ in dry $\mathrm{CH}_{2} \mathrm{Cl}_{2}(5 \mathrm{~mL})$, containing molecular sieves $4 \AA(0.2 \mathrm{~g})$, was stirred for $1 \mathrm{~h}$ under Ar. The mixture was cooled to $0{ }^{\circ} \mathrm{C}$ and TMSOTf $(6 \mu \mathrm{L}, 33$ $\mu \mathrm{mol})$ was added. The mixture was stirred for $1 \mathrm{~h}$, during which period the temperature was allowed to reach room temperature ( $\mathrm{rt}$ ), then neutralized with $\mathrm{Et}_{3} \mathrm{~N}$, washed with aq satd $\mathrm{NaHCO}_{3}$ and water, dried, filtered, and concentrated. Column chromatography (1:2 toluene-EtOAc) of the residue afforded 13, isolated as a white foam (148 mg, 45\%); $R_{f} 0.55$ (1:2 tolueneEtOAc); $[\alpha]_{\mathrm{D}}^{20}-6^{\circ}\left(c \quad 1, \mathrm{CHCl}_{3}\right) ;{ }^{1} \mathrm{H}$ NMR $(300 \mathrm{MHz}$, $\left.\mathrm{CDCl}_{3}\right): \delta 1.14-1.16\left(\mathrm{~m}, 4 \mathrm{H}, 2 \mathrm{CH}_{2}\right), 1.22-1.30(\mathrm{~m}, 2$ $\left.\mathrm{H}, \mathrm{CH}_{2}\right), 1.45-1.51\left(\mathrm{~m}, 2 \mathrm{H}, \mathrm{CH}_{2}\right), 1.95,2.04,2.05,2.07$, and $2.14\left(5 \mathrm{~s}, 3,3,9,3,3 \mathrm{H}, 7 \mathrm{COCH}_{3}\right), 2.27$ and $2.34(2 \mathrm{~s}$, each $\left.3 \mathrm{H}, 2 \mathrm{CH}_{3} \mathrm{C}_{6} \mathrm{H}_{4} \mathrm{CO}\right), 3.05\left(\mathrm{t}, 2 \mathrm{H}, \mathrm{CH}_{2} \mathrm{~N}_{3}\right), 3.49$ $(\mathrm{m}, 1 \mathrm{H}, \mathrm{OC} H \mathrm{H}), 3.60\left(\mathrm{~m}, 1 \mathrm{H}, \mathrm{H}-5^{\mathrm{II}}\right), 4.64\left(\mathrm{~d}, 1 \mathrm{H}, J_{1,2}\right.$ $\left.7.7 \mathrm{~Hz}, \mathrm{H}-1^{\mathrm{II}}\right), 4.90\left(\mathrm{t}, 1 \mathrm{H}, J_{2,3} 9.2 \mathrm{~Hz}, \mathrm{H}-2^{\mathrm{II}}\right), 4.95(\mathrm{dd}$, $\left.1 \mathrm{H}, J_{2,3} 10.3, J_{3,4} 3.4 \mathrm{~Hz}, \mathrm{H}-3^{\mathrm{III}}\right), 5.10\left(\mathrm{dd}, 1 \mathrm{H}, J_{1,2} 7.8\right.$ $\left.\mathrm{Hz}, \mathrm{H}-2^{\mathrm{III}}\right), 5.15\left(\mathrm{t}, 1 \mathrm{H}, \mathrm{H}-4^{\mathrm{I}}\right), 5.46\left(\mathrm{~d}, 1 \mathrm{H}, J_{1,2} 8.4 \mathrm{~Hz}\right.$, H-1 $\left.{ }^{\mathrm{I}}\right), 6.17\left(\mathrm{dd}, 1 \mathrm{H}, J_{2,3} 10.7, J_{3,4} 9.2 \mathrm{~Hz}, \mathrm{H}-3^{\mathrm{I}}\right), 7.04$ and 7.15 ( $2 \mathrm{~d}$, each $2 \mathrm{H}$, Phth), 7.61 and 7.78 ( $2 \mathrm{~d}$, each 4 $\left.\mathrm{H}, 2 \mathrm{CH}_{3} \mathrm{C}_{6} \mathrm{H}_{4} \mathrm{CO}\right) ;{ }^{13} \mathrm{C}$ NMR $\left(75.5 \mathrm{MHz}, \mathrm{CDCl}_{3}\right): \delta$ 20.3-20.6 $\left(\mathrm{COCH}_{3}\right), 21.4\left(2 \mathrm{CH}_{3} \mathrm{C}_{6} \mathrm{H}_{4} \mathrm{CO}\right), 25.3,26.0$, 28.4, and $28.9\left(4 \mathrm{CH}_{2}\right), 50.9\left(\mathrm{CH}_{2} \mathrm{~N}_{3}\right), 54.8\left(\mathrm{C}-2^{\mathrm{I}}\right), 60.6$, $62.0,68.1$, and $69.6\left(\mathrm{C}-6^{\mathrm{I}}, \mathrm{C}-6^{\mathrm{II}}, \mathrm{C}-6^{\mathrm{III}}, \mathrm{OCH}_{2}\right), 66.5$, $68.9,69.8,70.5,70.7,70.8,71.5,72.4,72.7,74.0$, and $76.2\left(\mathrm{C}-3^{\mathrm{I}}, \mathrm{C}-4^{\mathrm{I}}, \mathrm{C}-5^{\mathrm{I}}, \mathrm{C}-2^{\mathrm{II}}, \mathrm{C}-3^{\mathrm{II}}, \mathrm{C}-4^{\mathrm{II}}, \mathrm{C}-5^{\mathrm{II}}, \mathrm{C}-2^{\mathrm{III}}, \mathrm{C}-\right.$ $\left.3^{\mathrm{III}}, \mathrm{C}-4^{\mathrm{III}}, \mathrm{C}-5^{\mathrm{III}}\right), 98.0,100.3$, and $100.9\left(\mathrm{C}-1^{\mathrm{I}}, \mathrm{C}-1^{\mathrm{II}}, \mathrm{C}-\right.$ $\left.1^{\mathrm{III}}\right), 165.1$ and $165.4\left(2 \mathrm{CH}_{3} \mathrm{C}_{6} \mathrm{H}_{4} \mathrm{CO}\right), 168.8-170.1$ $\left(\mathrm{COCH}_{3}\right)$; Anal. Calcd for $\mathrm{C}_{62} \mathrm{H}_{72} \mathrm{~N}_{4} \mathrm{O}_{26}: \mathrm{C}, 57.76 ; \mathrm{H}$, 5.63; N, 4.35. Found: C, 57.65; H, 5.81; N, 4.19.

\subsection{6-Azidohexyl $(2,3,4,6$-tetra- $O$-acetyl- $\beta$-D- galactopyranosyl)-(1 $\rightarrow 4)-(2,3,6$-tri- $O$-acetyl- $\beta$-D- glucopyranosyl)-(1 $\rightarrow 6)$-2-acetamido-3,4-di- $O$-acetyl-2- deoxy- $\beta$-D-glucopyranoside (14)}

To a soln of $13(141 \mathrm{mg}, 0.11 \mathrm{mmol})$ in $\mathrm{MeOH}(20 \mathrm{~mL})$ and $\mathrm{CH}_{2} \mathrm{Cl}_{2}(3 \mathrm{~mL})$ was added $\mathrm{NaOMe}(\mathrm{pH} 10)$, and the mixture was stirred for $24 \mathrm{~h}$. After neutralization with Dowex $50 \times 8\left(\mathrm{H}^{+}\right)$and filtration, the solution was concentrated. To a solution of the residue in $1-\mathrm{BuOH}$ 
(45 mL) was added 1,2-diaminoethane ( $1 \mathrm{~mL}, 15 \mathrm{mmol})$, and the mixture was stirred overnight at $90{ }^{\circ} \mathrm{C}$, then, coconcentrated with toluene. A soln of the residue in Py $(50 \mathrm{~mL})$ and $\mathrm{Ac}_{2} \mathrm{O}(40 \mathrm{~mL})$ was stirred overnight, then co-concentrated with toluene, EtOH and $\mathrm{CH}_{2} \mathrm{Cl}_{2}$. Column chromatography $\left(3: 1 \mathrm{CH}_{2} \mathrm{Cl}_{2}\right.$-acetone) of the residue yielded 14, isolated as a colourless glass $(98 \mathrm{mg}$, $86 \%) ; R_{f} 0.61\left(9: 1 \mathrm{CH}_{2} \mathrm{Cl}_{2}-\mathrm{MeOH}\right) ;[\alpha]_{\mathrm{D}}^{20}-12^{\circ}(c 1$, $\left.\mathrm{CHCl}_{3}\right) ;{ }^{1} \mathrm{H}$ NMR $\left(500 \mathrm{MHz}, \mathrm{CDCl}_{3} ; 2 \mathrm{D}\right.$ TOCSY, ROESY, HSQC): $\delta$ 1.37-1.38 (m, $\left.4 \mathrm{H}, 2 \mathrm{CH}_{2}\right), 1.58-$ $1.59\left(\mathrm{~m}, 4 \mathrm{H}, 2 \mathrm{CH}_{2}\right), 1.93\left(\mathrm{~s}, 3 \mathrm{H}, \mathrm{NHCOCH}_{3}\right), 1.96$, 2.01, 2.03, 2.04, 2.06, 2.12, and $2.14(7 \mathrm{~s}, 3,6,3,6,3,3,3 \mathrm{H}$, $\left.9 \mathrm{COCH}_{3}\right), 3.26\left(\mathrm{t}, 2 \mathrm{H}, \mathrm{CH}_{2} \mathrm{~N}_{3}\right), 3.46(\mathrm{~m}, 1 \mathrm{H}, \mathrm{OCH} \mathrm{H})$, $3.70\left(\mathrm{~m}, 1 \mathrm{H}, \mathrm{H}-5^{\mathrm{I}}\right), 4.13\left(\mathrm{dd}, 1 \mathrm{H}, J_{5,6 \mathrm{~b}} 6.4, J_{6 \mathrm{a}, 6 \mathrm{~b}} 11.3\right.$ $\left.\mathrm{Hz}, \mathrm{H}-6 \mathrm{~b}^{\mathrm{III}}\right), 4.47\left(\mathrm{dd}, 1 \mathrm{H}, J_{5,6 \mathrm{a}} 2.2, J_{6 \mathrm{a}, 6 \mathrm{~b}} 12.2 \mathrm{~Hz}, \mathrm{H}-\right.$ $\left.6 \mathrm{a}^{\mathrm{II}}\right), 4.49\left(\mathrm{~d}, 1 \mathrm{H}, J_{1,2} 8.0 \mathrm{~Hz}, \mathrm{H}-1^{\mathrm{III}}\right), 4.58\left(\mathrm{~d}, 1 \mathrm{H}, J_{1,2}\right.$ $\left.7.6 \mathrm{~Hz}, \mathrm{H}-1^{\mathrm{II}}\right), 4.65\left(\mathrm{~d}, 1 \mathrm{H}, J_{1,2} 8.3 \mathrm{~Hz}, \mathrm{H}-1^{\mathrm{I}}\right), 4.95$ (dd, $\left.1 \mathrm{H}, J_{2,3} 10.4, J_{3,4} 3.4 \mathrm{~Hz}, \mathrm{H}-3^{\mathrm{III}}\right), 5.10\left(\mathrm{dd}, 1 \mathrm{H}, \mathrm{H}-2^{\mathrm{III}}\right)$, $5.14\left(\mathrm{t}, 1 \mathrm{H}, J_{2,3} 9.2, J_{3,4} 9.2 \mathrm{~Hz}, \mathrm{H}-3^{\mathrm{II}}\right), 5.26(\mathrm{dd}, 1 \mathrm{H}$, $\left.J_{2,3} 10.4, J_{3,4} 9.1 \mathrm{~Hz}, \mathrm{H}-3^{\mathrm{I}}\right), 5.34\left(\mathrm{~d}, 1 \mathrm{H}, J_{4,5}<1 \mathrm{~Hz}, \mathrm{H}-\right.$ $\left.4^{\mathrm{IIII}}\right), 5.79\left(\mathrm{~d}, 1 \mathrm{H}, J_{2, \mathrm{NH}} 8.9 \mathrm{~Hz}, \mathrm{NH} \mathrm{COCH}_{3}\right) ;{ }^{13} \mathrm{C} \mathrm{NMR}$ $\left(75.5 \mathrm{MHz}, \mathrm{CDCl}_{3}\right): \delta$ 20.2-20.5 $\left(\mathrm{COCH}_{3}\right), 22.9$ $\left(\mathrm{NHCOCH}_{3}\right), 25.2,26.1,28.5$, and $29.0\left(4 \mathrm{CH}_{2}\right), 51.0$ $\left(\mathrm{CH}_{2} \mathrm{~N}_{3}\right), 54.5\left(\mathrm{C}-2^{\mathrm{I}}\right), 60.6\left(\mathrm{C}-6^{\mathrm{III}}\right), 61.8\left(\mathrm{C}-6^{\mathrm{II}}\right), 66.4(\mathrm{C}-$ $\left.4^{\mathrm{III}}\right), 67.9\left(\mathrm{C}-6^{\mathrm{I}}\right), 68.8\left(\mathrm{C}-2^{\mathrm{III}}\right), 69.1\left(\mathrm{C}-4^{\mathrm{I}}\right), 69.2\left(\mathrm{OCH}_{2}\right)$, $70.3\left(\mathrm{C}-5^{\mathrm{III}}\right), 70.7\left(\mathrm{C}-3^{\mathrm{III}}\right), 71.3\left(\mathrm{C}-2^{\mathrm{II}}\right), 72.1\left(\mathrm{C}-3^{\mathrm{I}}\right), 72.4$ $\left(\mathrm{C}-5^{\mathrm{II}}\right), 72.6\left(\mathrm{C}-3^{\mathrm{II}}\right), 73.1\left(\mathrm{C}-5^{\mathrm{I}}\right), 75.9\left(\mathrm{C}-4^{\mathrm{II}}\right), 100.0(\mathrm{C}-$ $\left.1^{\mathrm{II}}\right), 100.2\left(\mathrm{C}-1^{\mathrm{I}}\right), 100.8\left(\mathrm{C}-1^{\mathrm{III}}\right), 168.7-170.4\left(\mathrm{COCH}_{3}\right.$, $\mathrm{NHCOCH}_{3}$ ); Anal. Calcd for $\mathrm{C}_{44} \mathrm{H}_{64} \mathrm{~N}_{4} \mathrm{O}_{25}$ : C, 50.38; $\mathrm{H}, 6.15$; N, 5.34. Found: C, 50.24; H, 6.06; N, 5.35; HRMS of $\mathrm{C}_{44} \mathrm{H}_{64} \mathrm{~N}_{4} \mathrm{O}_{25}(\mathrm{M}, 1048.386):[\mathrm{M}+\mathrm{H}]^{+}$found 1049.397, calcd 1049.393.

\subsection{6-Azidohexyl $\beta$-D-galactopyranosyl-( $1 \rightarrow 4)-\beta$-D- glucopyranosyl-(1 $\rightarrow 6)$-2-acetamido-2-deoxy- $\beta$-D- glucopyranoside (15)}

To a soln of $14(50 \mathrm{mg}, 47.6 \mu \mathrm{mol})$ in $\mathrm{MeOH}(8 \mathrm{~mL})$ and $\mathrm{CH}_{2} \mathrm{Cl}_{2}(1 \mathrm{~mL})$ was added $\mathrm{NaOMe}(\mathrm{pH} 10)$, and the mixture was stirred for $2.5 \mathrm{~h}$. After neutralization with Dowex $50 \times 8\left(\mathrm{H}^{+}\right)$and filtration, the solution was concentrated. Column chromatography $(2: 1 \mathrm{MeOH}-$ $\mathrm{CH}_{2} \mathrm{Cl}_{2}$ ) of the residue yielded $\mathbf{1 5}$, isolated as a colourless glass $(29 \mathrm{mg}, 90 \%) ; R_{f} 0.63\left(4: 1 \mathrm{MeOH}-\mathrm{CH}_{2} \mathrm{Cl}_{2}\right)$; $[\alpha]_{\mathrm{D}}^{20}-8^{\circ}(c 1$, water $) ;{ }^{13} \mathrm{C}$ NMR $\left(75.5 \mathrm{MHz}, \mathrm{D}_{2} \mathrm{O}\right): \delta$ $22.8\left(\mathrm{NDCOCH}_{3}\right), 25.3,26.2,28.6$, and $29.1\left(4 \mathrm{CH}_{2}\right)$, $51.8\left(\mathrm{CH}_{2} \mathrm{~N}_{3}\right), 56.2\left(\mathrm{C}-2^{\mathrm{I}}\right), 60.7\left(\mathrm{C}-6^{\mathrm{II}}\right), 61.7\left(\mathrm{C}-6^{\mathrm{III}}\right)$, $69.3\left(\mathrm{C}-6^{\mathrm{I}}\right), 71.1\left(\mathrm{OCH}_{2}\right), 69.2\left(\mathrm{C}-4^{\mathrm{III}}\right), 70.4,71.6,73.2$, $73.4,74.4,75.0,75.4,75.5,76.0$, and $79.1\left(\mathrm{C}-3^{\mathrm{I}}, \mathrm{C}-4^{\mathrm{I}}, \mathrm{C}-\right.$ $\left.5^{\mathrm{I}}, \mathrm{C}-2^{\mathrm{II}}, \mathrm{C}-3^{\mathrm{II}}, \mathrm{C}-4^{\mathrm{II}}, \mathrm{C}-5^{\mathrm{II}}, \mathrm{C}-2^{\mathrm{III}}, \mathrm{C}-3^{\mathrm{III}}, \mathrm{C}-5^{\mathrm{III}}\right), 101.8$ $\left(\mathrm{C}-1^{\mathrm{I}}\right), 103.2\left(\mathrm{C}-1^{\mathrm{II}}\right), 103.6\left(\mathrm{C}-1^{\mathrm{III}}\right), 175.0\left(\mathrm{NDCOCH}_{3}\right)$; HRMS of $\mathrm{C}_{26} \mathrm{H}_{46} \mathrm{~N}_{4} \mathrm{O}_{16}$ (M, 670.290): $\left[\mathrm{M}+\mathrm{NH}_{4}\right]^{+}$ found 688.330 , calcd 688.333. For ${ }^{1} \mathrm{H}$ NMR data, see Table 1.

\subsection{6-Aminohexyl $\beta$-D-galactopyranosyl-( $1 \rightarrow 4)-\beta$-D- glucopyranosyl-(1 $\rightarrow 6)$-2-acetamido-2-deoxy- $\beta$-D- glucopyranoside (3)}

To a soln of $15(60 \mathrm{mg}, 89.4 \mu \mathrm{mol})$ in $t$ - $\mathrm{BuOH}(25 \mathrm{~mL})$ and water $(20 \mathrm{~mL})$ were added $10 \% \mathrm{Pd}-\mathrm{C}(240 \mathrm{mg})$ and three drops aq $25 \% \mathrm{NH}_{3}$, and the mixture was stirred for $24 \mathrm{~h}$ under $\mathrm{H}_{2}$, then filtered over hyflo, and coconcentrated with toluene. Column chromatography of the residue on a Toyopearl HW-40S column, eluted with aq $0.1 \mathrm{M} \mathrm{NH}_{4} \mathrm{OAc}$, and subsequent lyophilization gave 3 , isolated as a white powder (41 $\mathrm{mg}, 71 \%) ; R_{f} 0.30$ $(2: 1: 1 \mathrm{AcOH}-1-\mathrm{BuOH}$-water $) ;[\alpha]_{\mathrm{D}}^{20}-2^{\circ}(c 1$, water $)$; ${ }^{13} \mathrm{C}$ NMR $\left(75.5 \mathrm{MHz}, \mathrm{D}_{2} \mathrm{O}\right): \delta 22.8\left(\mathrm{NDCOCH}_{3}\right), 25.2$, 25.8, 27.3, and $29.0\left(4 \mathrm{CH}_{2}\right), 40.0\left(\mathrm{CH}_{2} \mathrm{ND}_{2}\right), 56.1$ $\left(\mathrm{C}-2^{\mathrm{I}}\right), 60.7\left(\mathrm{C}-6^{\mathrm{II}}\right), 61.6\left(\mathrm{C}-6^{\mathrm{II}}\right), 69.1\left(\mathrm{C}-4^{\mathrm{III}}\right), 69.2$ $\left(\mathrm{C}-6^{\mathrm{I}}\right), 71.5\left(\mathrm{OCH}_{2}\right), 73.4\left(\mathrm{C}-2^{\mathrm{II}}\right), 70.3,71.5,73.1$, $74.3,74.9,75.4,75.5$, and $75.9\left(\mathrm{C}-3^{\mathrm{I}}, \mathrm{C}-4^{\mathrm{I}}, \mathrm{C}-5^{\mathrm{I}}, \mathrm{C}-3^{\mathrm{II}}\right.$, $\left.\mathrm{C}-5^{\mathrm{II}}, \mathrm{C}-2^{\mathrm{III}}, \mathrm{C}-3^{\mathrm{III}}, \mathrm{C}-5^{\mathrm{III}}\right), 79.0\left(\mathrm{C}-4^{\mathrm{II}}\right), 101.8\left(\mathrm{C}-1^{\mathrm{I}}\right)$, $103.2\left(\mathrm{C}-1^{\mathrm{II}}\right), \quad 103.5\left(\mathrm{C}-1^{\mathrm{III}}\right), \quad 175.0\left(\mathrm{NDCOCH}_{3}\right)$; HRMS of $\mathrm{C}_{26} \mathrm{H}_{48} \mathrm{~N}_{2} \mathrm{O}_{16} \quad(\mathrm{M}, \quad 644.300):[\mathrm{M}+\mathrm{H}]^{+}$ found 645.306, calcd 645.308. For ${ }^{1} \mathrm{H}$ NMR data, see Table 2.

\subsection{6-Aminohexyl $\beta$-D-galactopyranosyl-( $1 \rightarrow 4)-\beta$-D- glucopyranosyl-( $1 \rightarrow 6)$-[ $\beta$-D-galactopyranosyl-(1 $\rightarrow 4)]-2-$ acetamido-2-deoxy- $\beta$-D-glucopyranoside (1)}

To a soln of $3(8.4 \mathrm{mg}, 13.0 \mu \mathrm{mol})$ in aq $50 \mathrm{mM}$ sodium cacodylate buffer $\mathrm{pH} 7.5(600 \mu \mathrm{L})$, containing $5 \mathrm{mM}$ $\mathrm{MnCl}_{2}$, bovine serum albumin (BSA) $(0.5 \mathrm{mg})$, and $\mathrm{NaN}_{3}(0.02 \%)$, were added alkaline phosphatase (12 U), UDP-galactose $(15 \mathrm{mg}, 24.5 \mu \mathrm{mol})$, and $\beta$-1,4-galactosyltransferase $(2.5 \mathrm{U})$. The reaction mixture was incubated for $20 \mathrm{~h}$ at $37^{\circ} \mathrm{C}$, then, water $(150 \mu \mathrm{L})$ was added. UDP-Galactose was removed on a Dowex $1 \times 8\left(\mathrm{Cl}^{-}\right)$ column, eluted with water. The eluate was concentrated, and the residue applied on a Bio Gel P-2 column, eluted with aq $0.1 \mathrm{M} \mathrm{NH}_{4} \mathrm{HCO}_{3}$ at a flow rate of $40 \mathrm{~mL} / \mathrm{h}$. The appropriate fractions were freeze-dried to give $\mathbf{1}(9.6 \mathrm{mg}$, $91 \%) ; R_{f} 0.15$ (2:1:1 AcOH-1-BuOH-water); $[\alpha]_{\mathrm{D}}^{20}-3^{\circ}$ (c 0.8 , water); ${ }^{13} \mathrm{C}$ NMR $\left(75.5 \mathrm{MHz}, \mathrm{D}_{2} \mathrm{O}\right.$ ): $\delta 22.7$ $\left(\mathrm{NDCOCH}_{3}\right), 25.2,25.9,27.3$, and $29.0\left(4 \mathrm{CH}_{2}\right), 40.1$ $\left(\mathrm{CH}_{2} \mathrm{ND}_{2}\right), 55.8\left(\mathrm{C}-2^{\mathrm{I}}\right), 60.7,61.7(2 \mathrm{C}), 68.1$, and 71.2 $\left(\mathrm{C}-6^{\mathrm{I}}, \mathrm{C}-6^{\mathrm{II}}, \mathrm{C}-6^{\mathrm{III}}, \mathrm{C}-6^{\mathrm{IV}}, \mathrm{OCH}_{2}\right), 69.2(2 \mathrm{C}), 71.6(3 \mathrm{C})$, 73.0, 73.2, 73.3, 74.2, 75.0, 75.4, 75.9, 76.0, 78.7, and $79.1\left(\mathrm{C}-3^{\mathrm{I}}, \mathrm{C}-4^{\mathrm{I}}, \mathrm{C}-5^{\mathrm{I}}, \mathrm{C}-2^{\mathrm{II}}, \mathrm{C}-3^{\mathrm{II}}, \mathrm{C}-4^{\mathrm{II}}, \mathrm{C}-5^{\mathrm{II}}, \mathrm{C}-2^{\mathrm{III}}, \mathrm{C}-\right.$ $\left.3^{\mathrm{III}}, \mathrm{C}-4^{\mathrm{III}}, \mathrm{C}-5^{\mathrm{III}}, \mathrm{C}-2^{\mathrm{IV}}, \mathrm{C}-3^{\mathrm{IV}}, \mathrm{C}-4^{\mathrm{IV}}, \mathrm{C}-5^{\mathrm{IV}}\right), 101.8$, 103.0, 103.4, and $103.6\left(\mathrm{C}-1^{\mathrm{I}}, \mathrm{C}-1^{\mathrm{II}}, \mathrm{C}-1^{\mathrm{III}}, \mathrm{C}-1^{\mathrm{IV}}\right), 175.0$ $\left(\mathrm{NDCOCH}_{3}\right)$; HRMS of $\mathrm{C}_{32} \mathrm{H}_{58} \mathrm{~N}_{2} \mathrm{O}_{21}$ (M, 806.353): $[\mathrm{M}+\mathrm{H}]^{+}$found 807.359, calcd 807.361. For ${ }^{1} \mathrm{H}$ NMR data, see Table 3 . 
3.12. 6-Azidohexyl (2,6-di- $O$-acetyl-3,4-di- $O$ isopropylidene- $\beta$-D-galactopyranosyl)-( $1 \rightarrow 4)-(2,3,6$-tri$O$-acetyl- $\beta$-D-glucopyranosyl)-(1 $\rightarrow$ 6)-2-deoxy-3,4-di- $O$ $p$-methylbenzoyl-2-phthalimido- $\beta$-D-glucopyranoside (17)

A soln of (2,6-di- $O$-acetyl-3,4-di- $O$-isopropylidene- $\beta$-Dgalactopyranosyl)-( $1 \rightarrow 4)-2,3,6$-tri- $O$-acetyl- $\beta$-D-glucopyranosyl trichloroacetimidate donor $(\mathbf{1 6})^{23}(0.51 \mathrm{~g}, 0.69$ $\mathrm{mmol})$ and $11(0.31 \mathrm{~g}, 0.48 \mathrm{mmol})$ in dry $\mathrm{CH}_{2} \mathrm{Cl}_{2}(5 \mathrm{~mL})$, containing powdered molecular sieves $4 \AA$ ( $1 \mathrm{~g})$, was stirred for $1 \mathrm{~h}$ under Ar. After cooling to $0{ }^{\circ} \mathrm{C}$, TMSOTf $(83 \mu \mathrm{L}, 0.46 \mathrm{mmol})$ was added. The mixture was stirred for $20 \mathrm{~min}$, during which period the temperature was allowed to reach $\mathrm{rt}$, then neutralized with $\mathrm{Et}_{3} \mathrm{~N}$, filtered over hyflo, and concentrated. Column chromatography (3:1 toluene-EtOAc with $1 \% \mathrm{Et}_{3} \mathrm{~N}$ ) of the residue afforded 17, isolated as a white foam $(0.36 \mathrm{~g}, 62 \%) ; R_{f}$ $0.57\left(1: 1\right.$ toluene-EtOAc); $[\alpha]_{\mathrm{D}}^{20}+3^{\circ}\left(c 1, \mathrm{CHCl}_{3}\right) ;{ }^{1} \mathrm{H}$ NMR (300 MHz, $\left.\mathrm{CDCl}_{3}\right): \delta 1.15-1.16\left(\mathrm{~m}, 4 \mathrm{H}, 2 \mathrm{CH}_{2}\right)$, $1.26-1.30\left(\mathrm{~m}, 2 \mathrm{H}, \mathrm{CH}_{2}\right), 1.30$ and $1.52[2 \mathrm{~s}$, each $3 \mathrm{H}$, $\left.\mathrm{C}\left(\mathrm{CH}_{3}\right)_{2}\right], 1.47-1.52\left(\mathrm{~m}, 2 \mathrm{H}, \mathrm{CH}_{2}\right), 2.04,2.05,2.07$, and $2.11\left(4 \mathrm{~s}, 3,3,6,3 \mathrm{H}, 5 \mathrm{COCH}_{3}\right), 2.25$ and $2.32(2 \mathrm{~s}$, each 3 $\left.\mathrm{H}, 2 \mathrm{CH}_{3} \mathrm{C}_{6} \mathrm{H}_{4} \mathrm{CO}\right), 3.04\left(\mathrm{t}, 2 \mathrm{H}, \mathrm{CH}_{2} \mathrm{~N}_{3}\right), 3.49(\mathrm{~m}, 1 \mathrm{H}$, $\mathrm{OC} H \mathrm{H}), 3.60\left(\mathrm{~m}, 1 \mathrm{H}, \mathrm{H}-5^{\mathrm{II}}\right), 4.47\left(\mathrm{dd}, 1 \mathrm{H}, J_{1,2} 8.4, J_{2,3}\right.$ $\left.10.7 \mathrm{~Hz}, \mathrm{H}-2^{\mathrm{I}}\right), 4.63\left(\mathrm{~d}, 1 \mathrm{H}, J_{1,2} 7.8 \mathrm{~Hz}, \mathrm{H}-1^{\mathrm{II}}\right), 4.85(\mathrm{t}, 1$ $\left.\mathrm{H}, \mathrm{H}-2^{\mathrm{III}}\right), 4.93\left(\mathrm{t}, 1 \mathrm{H}, \mathrm{H}-2^{\mathrm{II}}\right), 5.15\left(\mathrm{t}, 1 \mathrm{H}, \mathrm{H}-3^{\mathrm{II}}\right), 5.36$ $\left(\mathrm{t}, 1 \mathrm{H}, \mathrm{H}-4^{\mathrm{I}}\right), 5.47\left(\mathrm{~d}, 1 \mathrm{H}, \mathrm{H}-1^{\mathrm{I}}\right), 6.18\left(\mathrm{dd}, 1 \mathrm{H}, J_{3,4} 9.2\right.$ $\left.\mathrm{Hz}, \mathrm{H}-3^{\mathrm{I}}\right), 7.03$ and $7.13(2 \mathrm{~d}$, each $2 \mathrm{H}, \mathrm{Phth}), 7.62$ and $7.78\left(2 \mathrm{~d}\right.$, each $\left.4 \mathrm{H}, 2 \mathrm{CH}_{3} \mathrm{C}_{6} \mathrm{H}_{4} \mathrm{CO}\right) ;{ }^{13} \mathrm{C}$ NMR $(75.5$ $\left.\mathrm{MHz}, \mathrm{CDCl}_{3}\right): \delta 20.5-20.6\left(\mathrm{COCH}_{3}\right), 21.3$ and $21.4(2$ $\left.\mathrm{CH}_{3} \mathrm{C}_{6} \mathrm{H}_{4} \mathrm{CO}\right), 25.2$ and $26.0\left(2 \mathrm{CH}_{2}\right), 25.9$ and 27.1 $\left[\left(\mathrm{CH}_{3}\right)_{2} \mathrm{C}\right], 28.4$ and $28.9\left(2 \mathrm{CH}_{2}\right), 50.9\left(\mathrm{CH}_{2} \mathrm{~N}_{3}\right), 54.7$ $\left(\mathrm{C}-2^{\mathrm{I}}\right), 62.1,62.9,68.1$, and $69.6\left(\mathrm{C}-6^{\mathrm{I}}, \mathrm{C}-6^{\mathrm{II}}, \mathrm{C}-6^{\mathrm{III}}\right.$, $\left.\mathrm{OCH}_{2}\right), 69.6,70.7$ (2 C), 71.4, 72.3, $72.6(2 \mathrm{C}), 72.9,74.0$, 75.9, and $76.7\left(\mathrm{C}-3^{\mathrm{I}}, \mathrm{C}-4^{\mathrm{I}}, \mathrm{C}-5^{\mathrm{I}}, \mathrm{C}-2^{\mathrm{II}}, \mathrm{C}-3^{\mathrm{II}}, \mathrm{C}-4^{\mathrm{II}}, \mathrm{C}-5^{\mathrm{II}}\right.$, $\left.\mathrm{C}-2^{\mathrm{III}}, \mathrm{C}-3^{\mathrm{III}}, \mathrm{C}-4^{\mathrm{III}}, \mathrm{C}-5^{\mathrm{III}}\right), 97.9,100.3$, and 100.5 (C-1 ${ }^{\mathrm{I}}$, $\left.\mathrm{C}-1^{\mathrm{II}}, \mathrm{C}-1^{\mathrm{III}}\right), 110.6\left[\left(\mathrm{CH}_{3}\right)_{2} \mathrm{C}\right], 165.1$ and 165.4 (2 $\left.\mathrm{CH}_{3} \mathrm{C}_{6} \mathrm{H}_{4} \mathrm{CO}\right), \quad 168.9-170.5\left(\mathrm{COCH}_{3}\right)$; HRMS of $\mathrm{C}_{61} \mathrm{H}_{72} \mathrm{~N}_{4} \mathrm{O}_{24} \quad\left(\mathrm{M}\right.$, 1244.453): $\left[\mathrm{M}+\mathrm{NH}_{4}\right]^{+}$found 1262.475, calcd 1262.488

\subsection{6-Azidohexyl (2,6-di- $O$-acetyl- $\beta$-D-} galactopyranosyl)-( $(1 \rightarrow 4)-(2,3,6$-tri- $O$-acetyl- $\beta$-Dglucopyranosyl)-(1 $\rightarrow 6)-2-$ deoxy-3,4-di- $O-p$ methylbenzoyl-2-phthalimido- $\beta$-D-glucopyranoside (18)

To a soln of $\mathbf{1 7}(155 \mathrm{mg}, 0.13 \mathrm{mmol})$ in $\mathrm{CH}_{2} \mathrm{Cl}_{2}(2.5 \mathrm{~mL})$ at $0{ }^{\circ} \mathrm{C}$, was added aq $90 \%$ TFA $(0.25 \mathrm{~mL})$. The mixture was stirred for $1.5 \mathrm{~h}$, then co-concentrated with toluene to give 18, isolated as a colourless glass $(127 \mathrm{mg}, 84 \%)$; $R_{f} 0.20$ (1:2 toluene-EtOAc); $[\alpha]_{\mathrm{D}}^{20}-6^{\circ}\left(c \quad 1, \mathrm{CHCl}_{3}\right)$; ${ }^{1} \mathrm{H}$ NMR (500 MHz, $\mathrm{CDCl}_{3} ; 2 \mathrm{D}$ TOCSY, ROESY): $\delta$ 1.11-1.17 (m, $\left.4 \mathrm{H}, 2 \mathrm{CH}_{2}\right), 1.25-1.30\left(\mathrm{~m}, 2 \mathrm{H}, \mathrm{CH}_{2}\right)$, 1.44-1.50 (m, $\left.2 \mathrm{H}, \mathrm{CH}_{2}\right), 2.04,2.07,2.10$, and $2.12(4 \mathrm{~s}$, $\left.6,3,3,3 \mathrm{H}, 5 \mathrm{COCH}_{3}\right), 2.28$ and $2.34(2 \mathrm{~s}$, each $3 \mathrm{H}, 2$ $\mathrm{CH}_{3} \mathrm{C}_{6} \mathrm{H}_{4} \mathrm{CO}$ ), 2.81 (bs, $\left.1 \mathrm{H}, \mathrm{OH}\right), 3.04$ (t, $2 \mathrm{H}, \mathrm{CH}_{2} \mathrm{~N}_{3}$ ),
3.24 (bs, $1 \mathrm{H}, \mathrm{OH}), 3.48(\mathrm{~m}, 1 \mathrm{H}, \mathrm{OC} H \mathrm{H}), 3.72(\mathrm{dd}, 1 \mathrm{H}$, $\left.J_{3,4} 9.3, J_{4,5} 9.7 \mathrm{~Hz}, \mathrm{H}-4^{\mathrm{II}}\right), 3.78\left(\mathrm{dd}, 1 \mathrm{H}, J_{5,6 \mathrm{~b}} 7.7, J_{6 \mathrm{a}, 6 \mathrm{~b}}\right.$ $\left.11.6 \mathrm{~Hz}, \mathrm{H}-6 \mathrm{~b}^{\mathrm{I}}\right), 3.83$ (bs, $\left.1 \mathrm{H}, \mathrm{H}-4^{\mathrm{III}}\right), 4.05(\mathrm{~m}, 1 \mathrm{H}, \mathrm{H}-$ $\left.5^{\mathrm{I}}\right), 4.13\left(\mathrm{dd}, 1 \mathrm{H}, J_{5,6 \mathrm{~b}} 5.7, J_{6 \mathrm{a}, 6 \mathrm{~b}} 11.9 \mathrm{~Hz}, \mathrm{H}-6 \mathrm{~b}^{\mathrm{II}}\right), 4.22$ (dd, $\left.1 \mathrm{H}, J_{5,6 \mathrm{a}} 6.4, J_{6 \mathrm{a}, 6 \mathrm{~b}} 11.4 \mathrm{~Hz}, \mathrm{H}-6 \mathrm{~b}^{\mathrm{III}}\right), 4.42(\mathrm{dd}, 1 \mathrm{H}$, $\left.J_{5,6 \mathrm{a}} 1.5 \mathrm{~Hz}, \mathrm{H}-6 \mathrm{a}^{\mathrm{II}}\right), 4.46\left(\mathrm{dd}, 1 \mathrm{H}, J_{1,2} 8.5, J_{2,3} 10.7 \mathrm{~Hz}\right.$, $\left.\mathrm{H}-2^{\mathrm{I}}\right), 4.62\left(\mathrm{~d}, 1 \mathrm{H}, J_{1,2} 7.9 \mathrm{~Hz}, \mathrm{H}-1^{\mathrm{II}}\right), 4.81\left(\mathrm{dd}, 1 \mathrm{H}, J_{1,2}\right.$ 8.0, $\left.J_{2,3} 9.1 \mathrm{~Hz}, \mathrm{H}-2^{\mathrm{III}}\right), 4.92\left(\mathrm{dd}, 1 \mathrm{H}, J_{2,3} 9.2 \mathrm{~Hz}, \mathrm{H}-2^{\mathrm{II}}\right)$, $5.13\left(\mathrm{t}, 1 \mathrm{H}, \mathrm{H}-3^{\mathrm{II}}\right), 5.34\left(\mathrm{dd}, 1 \mathrm{H}, J_{3,4} 9.3, J_{4,5} 9.9 \mathrm{~Hz}, \mathrm{H}-\right.$ $\left.4^{\mathrm{I}}\right), 5.45\left(\mathrm{~d}, 1 \mathrm{H}, \mathrm{H}-1^{\mathrm{I}}\right), 6.16\left(\mathrm{dd}, 1 \mathrm{H}, \mathrm{H}-3^{\mathrm{I}}\right), 7.04$ and 7.15 ( $2 \mathrm{~d}$, each $2 \mathrm{H}, \mathrm{Phth}), 7.62$ and 7.78 ( $2 \mathrm{~d}$, each $4 \mathrm{H}, 2$ $\left.\mathrm{CH}_{3} \mathrm{C}_{6} \mathrm{H}_{4} \mathrm{CO}\right) ;{ }^{13} \mathrm{C}$ NMR $\left(75.5 \mathrm{MHz}, \mathrm{CDCl}_{3}\right): \delta 20.4-$ $20.6\left(\mathrm{COCH}_{3}\right), 21.2$ and $21.3\left(2 \mathrm{CH}_{3} \mathrm{C}_{6} \mathrm{H}_{4} \mathrm{CO}\right), 25.1$, $25.9,28.3$, and $28.8\left(4 \mathrm{CH}_{2}\right), 50.8\left(\mathrm{CH}_{2} \mathrm{~N}_{3}\right), 54.6\left(\mathrm{C}-2^{\mathrm{I}}\right)$, $62.0,62.3,68.1$, and $69.4\left(\mathrm{C}-6^{\mathrm{I}}, \mathrm{C}-6^{\mathrm{II}}, \mathrm{C}-6^{\mathrm{III}}, \mathrm{OCH}_{2}\right)$, $68.3,69.8,70.7,71.2,72.1,72.2,72.5,72.7,73.7,76.1$, and $77.1\left(\mathrm{C}-3^{\mathrm{I}}, \mathrm{C}-4^{\mathrm{I}}, \mathrm{C}-5^{\mathrm{I}}, \mathrm{C}-2^{\mathrm{II}}, \mathrm{C}-3^{\mathrm{II}}, \mathrm{C}-4^{\mathrm{II}}, \mathrm{C}-5^{\mathrm{II}}, \mathrm{C}-\right.$ $\left.2^{\mathrm{III}}, \mathrm{C}-3^{\mathrm{III}}, \mathrm{C}-4^{\mathrm{III}}, \mathrm{C}-5^{\mathrm{III}}\right), 97.8,100.4$, and $100.7\left(\mathrm{C}-1^{\mathrm{I}}\right.$, $\left.\mathrm{C}-1^{\mathrm{II}}, \mathrm{C}-1^{\mathrm{III}}\right), 165.0$ and $165.3\left(2 \mathrm{CH}_{3} \mathrm{C}_{6} \mathrm{H}_{4} \mathrm{CO}\right), 169.1-$ $170.7\left(\mathrm{COCH}_{3}\right)$; HRMS of $\mathrm{C}_{58} \mathrm{H}_{68} \mathrm{~N}_{4} \mathrm{O}_{24}(\mathrm{M}, 1204.422)$ : $[\mathrm{M}+\mathrm{H}]^{+}$found 1205.399 , calcd 1205.430 .

\subsection{6-Azidohexyl $(2,4,6$-tri- $O$-acetyl- $\beta$-D-} galactopyranosyl)-( $(1 \rightarrow 4)-(2,3,6$-tri- $O$-acetyl- $\beta$-Dglucopyranosyl)-(1 $\rightarrow 6)-2$-deoxy-3,4-di- $O$ - $p$ methylbenzoyl-2-phthalimido- $\beta$-D-glucopyranoside (19)

To a soln of $\mathbf{1 8}(86 \mathrm{mg}, 72.8 \mu \mathrm{mol})$ in dry $\mathrm{MeCN}(1 \mathrm{~mL})$ were added trimethyl orthoacetate $(23 \mu \mathrm{L}, 182 \mu \mathrm{mol})$ and a catalytic amount of $p$-toluenesulfonic acid. After stirring for $3.5 \mathrm{~h}$, aq $80 \% \mathrm{AcOH}(50 \mu \mathrm{L})$ was added, and the stirring was continued for $1 \mathrm{~h}$. The mixture was diluted with $\mathrm{CH}_{2} \mathrm{Cl}_{2}$, washed with cold aq satd $\mathrm{NaHCO}_{3}$ and cold water, dried, filtered, and concentrated. Column chromatography (1:4 toluene-EtOAc) of the residue afforded 19 , isolated as a white foam $(78 \mathrm{mg}$, $85 \%) ; R_{f} 0.49$ (1:4 toluene-EtOAc); $[\alpha]_{\mathrm{D}}^{20}-10^{\circ}(c 1$, $\left.\mathrm{CHCl}_{3}\right) ;{ }^{1} \mathrm{H}$ NMR $\left(300 \mathrm{MHz}, \mathrm{CDCl}_{3}\right): \delta 1.14-1.16(\mathrm{~m}$, $\left.4 \mathrm{H}, 2 \mathrm{CH}_{2}\right), 1.23-1.28\left(\mathrm{~m}, 2 \mathrm{H}, \mathrm{CH}_{2}\right), 1.45-1.53(\mathrm{~m}, 2$ $\left.\mathrm{H}, \mathrm{CH}_{2}\right), 2.03,2.04,2.06,2.07,2.11$, and $2.15(6 \mathrm{~s}$, each 3 $\left.\mathrm{H}, 6 \mathrm{COCH}_{3}\right), 2.27$ and $2.34(2 \mathrm{~s}$, each $3 \mathrm{H}, 2$ $\left.\mathrm{CH}_{3} \mathrm{C}_{6} \mathrm{H}_{4} \mathrm{CO}\right), 2.76(\mathrm{bd}, 1 \mathrm{H}, \mathrm{OH}), 3.05$ (t, $2 \mathrm{H}$, $\left.\mathrm{CH}_{2} \mathrm{~N}_{3}\right), 3.49(\mathrm{~m}, 1 \mathrm{H}, \mathrm{OCH} \mathrm{H}), 3.61\left(\mathrm{~m}, 1 \mathrm{H}, \mathrm{H}-5^{\mathrm{II}}\right)$, $4.47\left(\mathrm{dd}, 1 \mathrm{H}, J_{1,2} 8.4, J_{2,3} 10.7 \mathrm{~Hz}, \mathrm{H}-2^{\mathrm{I}}\right), 4.63(\mathrm{~d}, 1 \mathrm{H}$, $\left.J_{1,2} 7.8 \mathrm{~Hz}, \mathrm{H}-1^{\mathrm{II}}\right), 4.85\left(\mathrm{dd}, 1 \mathrm{H}, J_{1,2} 7.8, J_{2,3} 9.9 \mathrm{~Hz}, \mathrm{H}-\right.$ $\left.2^{\mathrm{III}}\right), 4.92\left(\mathrm{dd}, 1 \mathrm{H}, J_{2,3} 9.3 \mathrm{~Hz}, \mathrm{H}-2^{\mathrm{II}}\right), 5.14(\mathrm{t}, 1 \mathrm{H}, \mathrm{H}-$ $\left.3^{\mathrm{II}}\right), 5.28\left(\mathrm{~d}, 1 \mathrm{H}, J_{3,4} 3.3, J_{4,5}<1 \mathrm{~Hz}, \mathrm{H}-4^{\mathrm{III}}\right), 5.35(\mathrm{t}, 1$ $\left.\mathrm{H}, \mathrm{H}-4^{\mathrm{I}}\right), 5.46\left(\mathrm{~d}, 1 \mathrm{H}, \mathrm{H}-1^{\mathrm{I}}\right), 6.18\left(\mathrm{dd}, 1 \mathrm{H}, J_{3,4} 9.1 \mathrm{~Hz}\right.$, H-3 $\left.{ }^{\mathrm{I}}\right), 7.04$ and $7.14(2 \mathrm{~d}$, each $2 \mathrm{H}, \mathrm{Phth}), 7.62$ and 7.78 $\left(2 \mathrm{~d}\right.$, each $\left.4 \mathrm{H}, 2 \mathrm{CH}_{3} \mathrm{C}_{6} \mathrm{H}_{4} \mathrm{CO}\right) ;{ }^{13} \mathrm{C}$ NMR $(75.5 \mathrm{MHz}$, $\left.\mathrm{CDCl}_{3}\right): \delta 20.5-20.6\left(\mathrm{COCH}_{3}\right), 21.4\left(2 \mathrm{CH}_{3} \mathrm{C}_{6} \mathrm{H}_{4} \mathrm{CO}\right)$, 25.3, 26.0, 28.4, and $28.9\left(4 \mathrm{CH}_{2}\right), 50.9\left(\mathrm{CH}_{2} \mathrm{~N}_{3}\right), 54.8$ $\left(\mathrm{C}-2^{\mathrm{I}}\right), 61.3,62.2,68.2$, and $69.6\left(\mathrm{C}-6^{\mathrm{I}}, \mathrm{C}-6^{\mathrm{II}}, \mathrm{C}-6^{\mathrm{III}}\right.$, $\left.\mathrm{OCH}_{2}\right), 69.1,69.9,70.7,70.8,71.4(2 \mathrm{C}), 72.5,72.6,72.8$, 74.0 , and $76.2\left(\mathrm{C}-3^{\mathrm{I}}, \mathrm{C}-4^{\mathrm{I}}, \mathrm{C}-5^{\mathrm{I}}, \mathrm{C}-2^{\mathrm{II}}, \mathrm{C}-3^{\mathrm{II}}, \mathrm{C}-4^{\mathrm{II}}, \mathrm{C}-5^{\mathrm{II}}\right.$, $\left.\mathrm{C}-2^{\mathrm{III}}, \mathrm{C}-3^{\mathrm{III}}, \mathrm{C}-4^{\mathrm{III}}, \mathrm{C}-5^{\mathrm{III}}\right), 165.1$ and 165.4 
$\left.\mathrm{CH}_{3} \mathrm{C}_{6} \mathrm{H}_{4} \mathrm{CO}\right), \quad 169.2-170.8\left(\mathrm{COCH}_{3}\right) ;$ HRMS of $\mathrm{C}_{60} \mathrm{H}_{70} \mathrm{~N}_{4} \mathrm{O}_{25} \quad(\mathrm{M}, \quad 1246.432):\left[\mathrm{M}+\mathrm{NH}_{4}\right]^{+}$found 1264.443, calcd 1264.467 .

\subsection{Benzyl (3,4,6-tri- $O$-acetyl-2-deoxy-2-phthalimido- $\beta$-D-glucopyranosyl)-(1 $\rightarrow 3)-(4-O$-acetyl-2,6-di- $O$-benzyl- $\beta$-D-galactopyranosyl)-( $1 \rightarrow 4)-2,3,6$-tri- $O$-benzyl- $\beta$-D- glucopyranoside (22)}

A soln of 3,4,6-tri- $O$-acetyl-2-deoxy-2-phthalimido- $\beta$-Dglucopyranosyl trichloroacetimidate $(5)^{20}(2.62 \mathrm{~g}, 4.52$ $\mathrm{mmol})$ and (4- $O$-acetyl-2,6-di- $O$-benzyl- $\beta$-D-galactopyranosyl)-( $1 \rightarrow 4)-1,2,3,6$-tetra- $O$-benzyl- $\beta$-D-glucopyranose $(\mathbf{2 1})^{25}$ (3.39 g, $\left.3.66 \mathrm{mmol}\right)$ in dry $\mathrm{CH}_{2} \mathrm{Cl}_{2}(80 \mathrm{~mL})$, containing powdered molecular sieves $4 \AA$ ( $2 \mathrm{~g})$, was stirred for $30 \mathrm{~min}$ under Ar. The mixture was cooled to $-70{ }^{\circ} \mathrm{C}$, and TMSOTf $(0.1 \mathrm{~mL}, 0.54 \mathrm{mmol})$ was added. The mixture was stirred for $2.5 \mathrm{~h}$, during which period the temperature was allowed to reach $\mathrm{rt}$, then neutralized with $\mathrm{Et}_{3} \mathrm{~N}$, and filtered over hyflo. The filtrate was washed with water, dried, filtered, and concentrated. Low-pressure column chromatography (9:1 tolueneEtOAc) of the residue gave 22, isolated as a white solid $(4.26 \mathrm{~g}, 86 \%) ; R_{f} 0.37\left(2: 1\right.$ toluene-EtOAc); $[\alpha]_{\mathrm{D}}^{20}+12^{\circ}$ (c $\left.1, \mathrm{CHCl}_{3}\right) ;{ }^{1} \mathrm{H}$ NMR $\left(500 \mathrm{MHz}, \mathrm{CDCl}_{3} ; 2 \mathrm{D}\right.$ TOCSY, ROESY, HSQC): $\delta 1.81,2.01,2.02$, and $2.06(4 \mathrm{~s}$, each 3 $\left.\mathrm{H}, 4 \mathrm{COCH}_{3}\right), 3.02\left(\mathrm{~m}, 1 \mathrm{H}, \mathrm{H}-5^{\mathrm{I}}\right), 3.46\left(\mathrm{t}, 1 \mathrm{H}, \mathrm{H}-5^{\mathrm{II}}\right)$, $3.52\left(\mathrm{dd}, 1 \mathrm{H}, J_{5,6 \mathrm{a}} 3.6, J_{6 \mathrm{a}, 6 \mathrm{~b}} 10.9 \mathrm{~Hz}, \mathrm{H}-6^{\mathrm{I}}\right), 3.60(\mathrm{dd}, 1$ $\left.\mathrm{H}, J_{2,3} 9.6, J_{3,4} 3.3 \mathrm{~Hz}, \mathrm{H}-3^{\mathrm{II}}\right), 3.81\left(\mathrm{~m}, 1 \mathrm{H}, \mathrm{H}-5^{\mathrm{III}}\right), 3.92$ $\left(\mathrm{m}, 1 \mathrm{H}, \mathrm{H}-4^{\mathrm{I}}\right), 4.22\left(\mathrm{dd}, 1 \mathrm{H}, J_{5,6 \mathrm{~b}} 4.1, J_{6 \mathrm{a}, 6 \mathrm{~b}} 12.3 \mathrm{~Hz}, \mathrm{H}-\right.$ $\left.6 \mathrm{~b}^{\mathrm{III}}\right), 4.32\left(\mathrm{~d}, 1 \mathrm{H}, J_{1,2} 7.4 \mathrm{~Hz}, \mathrm{H}-1^{\mathrm{II}}\right), 4.33\left(\mathrm{~d}, 1 \mathrm{H}, J_{1,2}\right.$ $\left.7.7 \mathrm{~Hz}, \mathrm{H}-1^{\mathrm{I}}\right), 4.15$ and $4.34\left(2 \mathrm{~d}\right.$, each $\left.1 \mathrm{H}, \mathrm{OCH}_{2} \mathrm{C}_{6} \mathrm{H}_{5}\right)$, $4.42\left(\mathrm{~d}, 1 \mathrm{H}, \mathrm{OCH} H \mathrm{C}_{6} \mathrm{H}_{5}\right), 4.51\left(\mathrm{~d}, 1 \mathrm{H}, \mathrm{OCH} H \mathrm{C}_{6} \mathrm{H}_{5}\right)$, 4.68 and $4.84\left(2 \mathrm{~d}\right.$, each $\left.1 \mathrm{H}, \mathrm{OCH}_{2} \mathrm{C}_{6} \mathrm{H}_{5}\right), 4.57$ and 4.86 $\left(2 \mathrm{~d}\right.$, each $\left.1 \mathrm{H}, \mathrm{OCH}_{2} \mathrm{C}_{6} \mathrm{H}_{5}\right), 4.65$ and $4.89(2 \mathrm{~d}$, each 1 $\left.\mathrm{H}, \mathrm{OCH}_{2} \mathrm{C}_{6} \mathrm{H}_{5}\right), 5.17\left(\mathrm{t}, 1 \mathrm{H}, J_{4,5} 9.5 \mathrm{~Hz}, \mathrm{H}-4^{\mathrm{III}}\right), 5.40(\mathrm{~d}$, $\left.1 \mathrm{H}, J_{4,5}<1 \mathrm{~Hz}, \mathrm{H}-4^{\mathrm{II}}\right), 5.55\left(\mathrm{~d}, 1 \mathrm{H}, J_{1,2} 8.5 \mathrm{~Hz}, \mathrm{H}-1^{\mathrm{III}}\right)$, $5.80\left(\mathrm{dd}, 1 \mathrm{H}, J_{2,3} 10.7, J_{3,4} 9.5 \mathrm{~Hz}, \mathrm{H}-3^{\mathrm{III}}\right) ;{ }^{13} \mathrm{C}$ NMR $\left(75.5 \mathrm{MHz}, \mathrm{CDCl}_{3}\right): \delta$ 19.9-20.2 $\left(\mathrm{COCH}_{3}\right), 54.5(\mathrm{C}-$ $\left.2^{\mathrm{III}}\right), 61.1\left(\mathrm{C}-6^{\mathrm{III}}\right), 67.3$ and $67.9\left(\mathrm{C}-6^{\mathrm{I}}, \mathrm{C}-6^{\mathrm{II}}\right), 70.4,72.7$, 73.0, 74.0, 74.5, and $74.7\left(6 \mathrm{OCH}_{2} \mathrm{C}_{6} \mathrm{H}_{5}\right), 68.4,69.3$, 70.1, 71.3, 72.2, 74.3, 75.2, 78.4, 78.9, 81.2, and 82.2 (C$2^{\mathrm{I}}, \mathrm{C}-3^{\mathrm{I}}, \mathrm{C}-4^{\mathrm{I}}, \mathrm{C}-5^{\mathrm{I}}, \mathrm{C}-2^{\mathrm{II}}, \mathrm{C}-3^{\mathrm{II}}, \mathrm{C}-4^{\mathrm{II}}, \mathrm{C}-5^{\mathrm{II}}, \mathrm{C}-3^{\mathrm{III}}, \mathrm{C}-$ $\left.4^{\mathrm{III}}, \mathrm{C}-5^{\mathrm{III}}\right), 97.8\left(\mathrm{C}-1^{\mathrm{III}}\right), 101.3$ and $101.9\left(\mathrm{C}-1^{\mathrm{I}}, \mathrm{C}-1^{\mathrm{II}}\right)$, $168.9,169.1,169.5$, and $170.1\left(4 \mathrm{COCH}_{3}\right)$; HRMS of $\mathrm{C}_{76} \mathrm{H}_{79} \mathrm{NO}_{21} \quad\left(\mathrm{M}\right.$, 1341.514): $\left[\mathrm{M}+\mathrm{NH}_{4}\right]^{+}$found 1359.546, calcd 1359.549.

\subsection{Benzyl (2-deoxy-2-phthalimido- $\beta$-D-} glucopyranosyl)-( $\rightarrow 3)$-(4- $O$-acetyl-2,6-di- $O$-benzyl- $\beta$-Dgalactopyranosyl)-( $1 \rightarrow 4)-2,3,6$-tri- $O$-benzyl- $\beta$-Dglucopyranoside (23)

To a soln of $22(6.88 \mathrm{~g}, 5.12 \mathrm{mmol})$ in $\mathrm{CH}_{2} \mathrm{Cl}_{2}(13.5 \mathrm{~mL})$ and $\mathrm{MeOH}(54 \mathrm{~mL})$ was added $\mathrm{NaOMe}(\mathrm{pH}$ 9). The mixture was stirred for $2 \mathrm{~h}$, then neutralized with Dowex
$50 \times 8\left(\mathrm{H}^{+}\right)$, filtered, and concentrated. Low-pressure column chromatography $(9: 1 \rightarrow 1: 1$ toluene-EtOAc) of the residue gave $\mathbf{2 3}$, isolated as a white solid $(5.1 \mathrm{~g}, 82 \%)$; $R_{f} 0.10\left(1: 1\right.$ toluene-EtOAc); $[\alpha]_{\mathrm{D}}^{20}+1^{\circ}\left(c 1, \mathrm{CHCl}_{3}\right) ;{ }^{1} \mathrm{H}$ NMR $\left(500 \mathrm{MHz}, \mathrm{CDCl}_{3} ; 2 \mathrm{D}\right.$ TOCSY, ROESY, HSQC): $\delta 2.07\left(\mathrm{~s}, 3 \mathrm{H}, \mathrm{COCH}_{3}\right), 2.97\left(\mathrm{~m}, 1 \mathrm{H}, \mathrm{H}-5^{\mathrm{I}}\right)$, $3.23\left(\mathrm{dd}, 1 \mathrm{H}, J_{5,6 \mathrm{~b}} 7.4, J_{6 \mathrm{a}, 6 \mathrm{~b}} 9.6 \mathrm{~Hz}, \mathrm{H}-6 \mathrm{~b}^{\mathrm{II}}\right), 3.68(\mathrm{t}, 1$ $\left.\mathrm{H}, \mathrm{H}-4^{\mathrm{III}}\right), 3.81\left(\mathrm{dd}, 1 \mathrm{H}, J_{5,6 \mathrm{~b}} 3.1, J_{6 \mathrm{a}, 6 \mathrm{~b}} 12.3 \mathrm{~Hz}, \mathrm{H}-\right.$ $\left.6 \mathrm{~b}^{\mathrm{III}}\right), 3.87\left(\mathrm{dd}, 1 \mathrm{H}, \mathrm{H}-4^{\mathrm{I}}\right), 3.92\left(\mathrm{dd}, 1 \mathrm{H}, J_{5,6 \mathrm{a}} 2.0 \mathrm{~Hz}\right.$, H-6a $\left.{ }^{\mathrm{III}}\right), 4.04\left(\mathrm{dd}, 1 \mathrm{H}, J_{1,2} 8.1, J_{2,3} 10.6 \mathrm{~Hz}, \mathrm{H}-2^{\mathrm{III}}\right), 4.08$ and $4.22\left(2 \mathrm{~d}\right.$, each $\left.1 \mathrm{H}, \mathrm{OCH}_{2} \mathrm{C}_{6} \mathrm{H}_{5}\right), 4.24$ and $4.38(2 \mathrm{~d}$, each $\left.1 \mathrm{H}, \mathrm{OCH}_{2} \mathrm{C}_{6} \mathrm{H}_{5}\right), 4.24$ and $4.45(2 \mathrm{~d}$, each $1 \mathrm{H}$, $\left.\mathrm{OCH}_{2} \mathrm{C}_{6} \mathrm{H}_{5}\right), 4.66$ and $4.83\left(2 \mathrm{~d}\right.$, each $\left.1 \mathrm{H}, \mathrm{OCH}_{2} \mathrm{C}_{6} \mathrm{H}_{5}\right)$, 4.55 and $4.85\left(2 \mathrm{~d}\right.$, each $\left.1 \mathrm{H}, \mathrm{OCH}_{2} \mathrm{C}_{6} \mathrm{H}_{5}\right), 4.63$ and 4.87 $\left(2 \mathrm{~d}\right.$, each $\left.1 \mathrm{H}, \mathrm{OCH}_{2} \mathrm{C}_{6} \mathrm{H}_{5}\right), 5.39\left(\mathrm{~d}, 1 \mathrm{H}, \mathrm{H}-1^{\mathrm{III}}\right), 5.53$ $\left(\mathrm{d}, 1 \mathrm{H}, J_{3,4} 3.5, J_{4,5}<1 \mathrm{~Hz}, \mathrm{H}-4{ }^{\mathrm{II}}\right) ;{ }^{13} \mathrm{C}$ NMR $(75.5$ $\left.\mathrm{MHz}, \mathrm{CDCl}_{3}\right): \delta 20.6\left(\mathrm{COCH}_{3}\right), 56.6\left(\mathrm{C}-2^{\mathrm{III}}\right), 61.0(\mathrm{C}-$ $\left.6^{\mathrm{III}}\right), 67.3$ and $67.7\left(\mathrm{C}-6^{\mathrm{I}}, \mathrm{C}-6^{\mathrm{II}}\right), 70.4,72.7,73.1,74.0$, 74.6, and $74.7\left(6 \mathrm{OCH}_{2} \mathrm{C}_{6} \mathrm{H}_{5}\right), 70.2(2 \mathrm{C}), 70.6,71.8$, 74.3, 75.5 (2 C), 78.2, 80.1, 81.2, and 82.3 (C-2 ${ }^{\mathrm{I}}, \mathrm{C}-3^{\mathrm{I}}$, C$\left.4^{\mathrm{I}}, \mathrm{C}-5^{\mathrm{I}}, \mathrm{C}-2^{\mathrm{II}}, \mathrm{C}-3^{\mathrm{II}}, \mathrm{C}-4^{\mathrm{II}}, \mathrm{C}-5^{\mathrm{II}}, \mathrm{C}-3^{\mathrm{III}}, \mathrm{C}-4^{\mathrm{III}}, \mathrm{C}-5^{\mathrm{III}}\right)$, $99.0\left(\mathrm{C}-1^{\mathrm{III}}\right), 101.4$ and $101.9\left(\mathrm{C}-1^{\mathrm{I}}, \mathrm{C}-1^{\mathrm{II}}\right), 170.9$ $\left(\mathrm{COCH}_{3}\right)$; HRMS of $\mathrm{C}_{70} \mathrm{H}_{73} \mathrm{NO}_{18}(\mathrm{M}, 1215.482)$ : $\left[\mathrm{M}+\mathrm{NH}_{4}\right]^{+}$found 1233.497, calcd 1233.517.

\subsection{Benzyl (6-O-tert-butyldiphenylsilyl-2-deoxy-2- phthalimido- $\beta$-D-glucopyranosyl)-( $\rightarrow 3)-(4-O$-acetyl-2,6- di- $O$-benzyl- $\beta$-D-galactopyranosyl)-( $1 \rightarrow 4)-2,3,6$-tri- $O$ - benzyl- $\beta$-D-glucopyranoside (24)}

To a soln of $23(4.25 \mathrm{~g}, 3.49 \mathrm{mmol})$ in dry $\mathrm{CH}_{2} \mathrm{Cl}_{2}(35$ $\mathrm{mL})$ was added dry Py $(1.67 \mathrm{~mL})$, 4-dimethylaminopyridine $(0.14 \mathrm{~g}, 1.1 \mathrm{mmol})$, tert -butyldiphenylsilylchloride $(0.61 \mathrm{~mL}, 4.35 \mathrm{mmol})$, and $\mathrm{Et}_{3} \mathrm{~N}(1.20 \mathrm{~mL})$. The mixture was stirred for $48 \mathrm{~h}$, washed with cold water, cold aq satd $\mathrm{NaHCO}_{3}$, and cold water, dried, filtered, and concentrated. Low-pressure column chromatography $(17: 3 \rightarrow 3: 1$ toluene-EtOAc) of the residue gave $\mathbf{2 4}$, isolated as a white solid $(4.51 \mathrm{~g}, 89 \%) ; R_{f} 0.49(1: 1$ toluene-EtOAc); $[\alpha]_{\mathrm{D}}^{20}-15^{\circ}\left(\mathrm{c} 1, \mathrm{CHCl}_{3}\right) ;{ }^{1} \mathrm{H}$ NMR (500 $\mathrm{MHz}, \mathrm{CDCl}_{3} ; 2 \mathrm{D}$ TOCSY, ROESY, HSQC): $\delta$ 1.05 [s, $9 \mathrm{H},\left(\mathrm{CH}_{3}\right)_{3} \mathrm{CSi}$ ] $1.97\left(\mathrm{~s}, 3 \mathrm{H}, \mathrm{COCH}_{3}\right), 3.03(\mathrm{~m}$, $\left.1 \mathrm{H}, \mathrm{H}-5^{\mathrm{I}}\right), 3.51\left(\mathrm{dd}, 1 \mathrm{H}, J_{5,6 \mathrm{~b}} 4.0, J_{6 \mathrm{a}, 6 \mathrm{~b}} 10.8 \mathrm{~Hz}, \mathrm{H}-\right.$ $\left.6 \mathrm{~b}^{\mathrm{I}}\right), 3.63\left(\mathrm{dd}, 1 \mathrm{H}, J_{2,3} 9.6, J_{3,4} 3.5 \mathrm{~Hz}, \mathrm{H}-3^{\mathrm{II}}\right), 3.89(\mathrm{~m}, 1$ $\left.\mathrm{H}, \mathrm{H}-4^{\mathrm{I}}\right), 3.98\left(\mathrm{~d}, 2 \mathrm{H}, \mathrm{H}-6 \mathrm{a}^{\mathrm{III}}, \mathrm{H}-6 \mathrm{~b}^{\mathrm{III}}\right), 4.05(\mathrm{dd}, 1 \mathrm{H}$, $\left.J_{1,2} 8.3, J_{2,3} 11.0 \mathrm{~Hz}, \mathrm{H}-2^{\mathrm{III}}\right), 4.28\left(\mathrm{~d}, 1 \mathrm{H}, J_{1,2} 7.2 \mathrm{~Hz}, \mathrm{H}-\right.$ $\left.1^{\mathrm{II}}\right), 4.12$ and $4.28\left(2 \mathrm{~d}\right.$, each $\left.1 \mathrm{H}, \mathrm{OCH}_{2} \mathrm{C}_{6} \mathrm{H}_{5}\right), 4.34(\mathrm{~d}, 1$ $\left.\mathrm{H}, J_{1,2} 7.4 \mathrm{~Hz}, \mathrm{H}-1^{\mathrm{I}}\right), 4.16$ and $4.36(2 \mathrm{~d}$, each $1 \mathrm{H}$, $\left.\mathrm{OCH}_{2} \mathrm{C}_{6} \mathrm{H}_{5}\right), 4.40\left(\mathrm{dd}, 1 \mathrm{H}, J_{3,4} 8.2 \mathrm{~Hz}, \mathrm{H}-3^{\mathrm{III}}\right), 4.25$ and $4.46\left(2 \mathrm{~d}\right.$, each $\left.1 \mathrm{H}, \mathrm{OCH}_{2} \mathrm{C}_{6} \mathrm{H}_{5}\right), 4.66$ and $4.83(2 \mathrm{~d}$, each $\left.1 \mathrm{H}, \mathrm{OCH}_{2} \mathrm{C}_{6} \mathrm{H}_{5}\right), 4.56$ and $4.86(2 \mathrm{~d}$, each $1 \mathrm{H}$, $\left.\mathrm{OCH}_{2} \mathrm{C}_{6} \mathrm{H}_{5}\right), 4.61$ and $4.87\left(2 \mathrm{~d}\right.$, each $\left.1 \mathrm{H}, \mathrm{OCH}_{2} \mathrm{C}_{6} \mathrm{H}_{5}\right)$; ${ }^{13} \mathrm{C}$ NMR $\left(75.5 \mathrm{MHz}, \mathrm{CDCl}_{3}\right): \delta 19.0\left[\left(\mathrm{CH}_{3}\right)_{3} \mathrm{CSi}\right], 20.5$ $\left(\mathrm{COCH}_{3}\right), 26.6\left[\left(\mathrm{CH}_{3}\right)_{3} \mathrm{CSi}\right], 56.6\left(\mathrm{C}-2^{\mathrm{III}}\right), 64.8\left(\mathrm{C}-6^{\mathrm{III}}\right)$, 67.6 and $68.1\left(\mathrm{C}-6^{\mathrm{I}}, \mathrm{C}-6^{\mathrm{II}}\right), 70.7,72.9,73.2,74.3,74.8$, and $74.9\left(6 \mathrm{OCH}_{2} \mathrm{C}_{6} \mathrm{H}_{5}\right), 70.1,70.9,72.5,74.0,74.4$, 
74.7, 75.5, 77.2, 79.1, 81.5, and $82.5\left(\mathrm{C}-2^{\mathrm{I}}, \mathrm{C}-3^{\mathrm{I}}, \mathrm{C}-4^{\mathrm{I}}, \mathrm{C}-\right.$ $\left.5^{\mathrm{I}}, \mathrm{C}-2^{\mathrm{II}}, \mathrm{C}-3^{\mathrm{II}}, \mathrm{C}-4^{\mathrm{II}}, \mathrm{C}-5^{\mathrm{II}}, \mathrm{C}-3^{\mathrm{III}}, \mathrm{C}-4^{\mathrm{III}}, \mathrm{C}-5^{\mathrm{III}}\right), 98.3$ $\left(\mathrm{C}-1^{\mathrm{III}}\right), 101.7$ and $102.2\left(\mathrm{C}-1^{\mathrm{I}}, \mathrm{C}-1^{\mathrm{II}}\right), 169.7\left(\mathrm{COCH}_{3}\right)$; HRMS of $\mathrm{C}_{86} \mathrm{H}_{91} \mathrm{NO}_{18} \mathrm{Si}(\mathrm{M}, 1453.600):\left[\mathrm{M}+\mathrm{NH}_{4}\right]^{+}$ found 1471.598 , calcd 1471.635 .

3.18. Benzyl (6-O-tert-butyldiphenylsilyl-2-deoxy-3,4-di$O$-p-methylbenzoyl-2-phthalimido- $\beta$-D-glucopyranosyl)$(1 \rightarrow 3)-(4-O$-acetyl-2,6-di- $O$-benzyl- $\beta$-Dgalactopyranosyl)-( $\rightarrow 4$ )-2,3,6-tri- $O$-benzyl- $\beta$-Dglucopyranoside (25)

To a soln of $24(1.17 \mathrm{~g}, 0.80 \mathrm{mmol})$ in dry Py $(115 \mathrm{~mL})$ was added dropwise at $0{ }^{\circ} \mathrm{C}$, a soln of $p$-methylbenzoyl chloride $(0.45 \mathrm{~mL}, 3.40 \mathrm{mmol})$ in dry $\mathrm{CH}_{2} \mathrm{Cl}_{2}(15 \mathrm{~mL})$. The mixture was stirred under $\mathrm{Ar}$ for $20 \mathrm{~h}$ at rt, diluted with $\mathrm{CH}_{2} \mathrm{Cl}_{2}$ and poured into cold water. The organic layer was washed with aq satd $\mathrm{NaHCO}_{3}$, dried, filtered, and concentrated. Low-pressure column chromatography (24:1 toluene-EtOAc) of the residue gave 25, isolated as a white solid $(1.01 \mathrm{~g}, 74 \%) ; R_{f} 0.57(9: 1$ toluene-EtOAc); $[\alpha]_{\mathrm{D}}^{20}+11^{\circ}\left(c \quad 1, \mathrm{CHCl}_{3}\right) ;{ }^{1} \mathrm{H}$ NMR $\left(500 \mathrm{MHz}, \mathrm{CDCl}_{3} ; 2 \mathrm{D}\right.$ TOCSY, ROESY, HSQC): $\delta$ $1.03\left[\mathrm{~s}, 9 \mathrm{H},\left(\mathrm{CH}_{3}\right)_{3} \mathrm{CSi}\right.$ ], $2.06\left(\mathrm{~s}, 3 \mathrm{H}, \mathrm{COCH}_{3}\right), 2.21$ and $2.31\left(2 \mathrm{~s}\right.$, each $\left.3 \mathrm{H}, 2 \mathrm{CH}_{3} \mathrm{C}_{6} \mathrm{H}_{4} \mathrm{CO}\right), 3.08\left(\mathrm{~m}, 1 \mathrm{H}, \mathrm{H}-5^{\mathrm{I}}\right)$, $3.33\left(\mathrm{dd}, 1 \mathrm{H}, J_{5,6 \mathrm{~b}} 6.4, J_{6 \mathrm{a}, 6 \mathrm{~b}} 9.9 \mathrm{~Hz}, \mathrm{H}-6 \mathrm{~b}^{\mathrm{II}}\right), 3.37$ (dd, 1 $\left.\mathrm{H}, J_{1,2} 8.0, J_{2,3} 9.5 \mathrm{~Hz}, \mathrm{H}-2^{\mathrm{II}}\right), 3.46\left(\mathrm{dd}, 1 \mathrm{H}, J_{5,6 \mathrm{a}}<1\right.$, $\left.J_{6 \mathrm{a}, 6 \mathrm{~b}} 11.0 \mathrm{~Hz}, \mathrm{H}-6 \mathrm{a}^{\mathrm{I}}\right), 3.53\left(\mathrm{t}, 1 \mathrm{H}, \mathrm{H}-5^{\mathrm{II}}\right), 3.58(\mathrm{dd}, 1 \mathrm{H}$, $\left.J_{5,6 \mathrm{~b}} 4.0 \mathrm{~Hz}, \mathrm{H}-6 \mathrm{~b}^{\mathrm{I}}\right), 4.13$ and $4.36(2 \mathrm{~d}$, each $1 \mathrm{H}$, $\left.\mathrm{OCH}_{2} \mathrm{C}_{6} \mathrm{H}_{5}\right), 4.21$ and $4.43\left(2 \mathrm{~d}\right.$, each $\left.1 \mathrm{H}, \mathrm{OCH}_{2} \mathrm{C}_{6} \mathrm{H}_{5}\right)$, 4.31 and $4.50\left(2 \mathrm{~d}\right.$, each $\left.1 \mathrm{H}, \mathrm{OCH}_{2} \mathrm{C}_{6} \mathrm{H}_{5}\right), 4.51(\mathrm{dd}, 1 \mathrm{H}$, $\left.J_{1,2} 8.1, J_{2,3} 10.7 \mathrm{~Hz}, \mathrm{H}-2^{\mathrm{III}}\right), 4.70$ and $4.87(2 \mathrm{~d}$, each 1 $\left.\mathrm{H}, \mathrm{OCH}_{2} \mathrm{C}_{6} \mathrm{H}_{5}\right), 4.59$ and $4.88(2 \mathrm{~d}$, each $1 \mathrm{H}$, $\left.\mathrm{OCH}_{2} \mathrm{C}_{6} \mathrm{H}_{5}\right), 4.65$ and $4.93\left(2 \mathrm{~d}\right.$, each $\left.1 \mathrm{H}, \mathrm{OCH}_{2} \mathrm{C}_{6} \mathrm{H}_{5}\right)$, $5.74\left(\mathrm{~d}, 1 \mathrm{H}, \mathrm{H}-1^{\mathrm{III}}\right), 6.29$ (dd, $\left.1 \mathrm{H}, J_{3,4} 9.2 \mathrm{~Hz}, \mathrm{H}-3^{\mathrm{III}}\right)$; ${ }^{13} \mathrm{C}$ NMR (75.5 MHz, $\left.\mathrm{CDCl}_{3}\right): \delta 18.9\left[\left(\mathrm{CH}_{3}\right)_{3} \mathrm{CSi}\right], 20.5$ $\left(\mathrm{COCH}_{3}\right), \quad 21.3$ and $21.4 \quad\left(2 \mathrm{CH}_{3} \mathrm{C}_{6} \mathrm{H}_{4} \mathrm{CO}\right), \quad 26.5$ $\left[\left(\mathrm{CH}_{3}\right)_{3} \mathrm{CSi}\right], 55.4\left(\mathrm{C}-2^{\mathrm{III}}\right), 62.8\left(\mathrm{C}-6^{\mathrm{III}}\right), 67.6\left(\mathrm{C}-6^{\mathrm{I}}\right)$, $68.1\left(\mathrm{C}-6^{\mathrm{II}}\right), 70.6,72.9,73.2,74.3,74.8$, and $74.9(6$ $\left.\mathrm{OCH}_{2} \mathrm{C}_{6} \mathrm{H}_{5}\right), 69.7\left(\mathrm{C}-4^{\mathrm{III}}\right), 70.2\left(\mathrm{C}-4^{\mathrm{II}}\right), 70.7\left(\mathrm{C}-3^{\mathrm{III}}\right)$, $72.7\left(\mathrm{C}-5^{\mathrm{II}}\right), 74.7\left(\mathrm{C}-5^{\mathrm{I}}\right), 74.8$ and $75.4\left(\mathrm{C}-4^{\mathrm{I}}, \mathrm{C}-5^{\mathrm{III}}\right), 77.2$ $\left(\mathrm{C}-3^{\mathrm{II}}\right), 79.2\left(\mathrm{C}-2^{\mathrm{II}}\right), 81.5$ and $82.5\left(\mathrm{C}-2^{\mathrm{I}}, \mathrm{C}-3^{\mathrm{I}}\right), 98.4(\mathrm{C}-$ $\left.1^{\mathrm{III}}\right), 101.8$ and $102.2\left(\mathrm{C}-1^{\mathrm{I}}, \mathrm{C}-1^{\mathrm{II}}\right), 164.8$ and $165.4(2$ $\left.\mathrm{CH}_{3} \mathrm{C}_{6} \mathrm{H}_{4} \mathrm{CO}\right), \quad 169.3 \quad\left(\mathrm{COCH}_{3}\right) ; \quad$ HRMS of $\mathrm{C}_{102} \mathrm{H}_{103} \mathrm{NO}_{20} \mathrm{Si} \quad(\mathrm{M}, 1689.684):\left[\mathrm{M}+\mathrm{NH}_{4}\right]^{+}$found 1707.713, calcd 1707.718.

3.19. (6-O-Tert-butyldiphenylsilyl-2-deoxy-3,4-di-O-pmethylbenzoyl-2-phthalimido- $\beta$-D-glucopyranosyl)-( $1 \rightarrow$ $3)-(2,4,6$-tri- $O$-acetyl- $\beta$-D-galactopyranosyl)-( $1 \rightarrow 4)$ 2,3,6-tri- $O$-acetyl- $\alpha, \beta$-D-glucopyranose (27)

To a soln of $25(1.46 \mathrm{~g}, 0.87 \mathrm{mmol})$ in EtOH $(45 \mathrm{~mL})$ and EtOAc $(45 \mathrm{~mL})$ was added $10 \% \mathrm{Pd}-\mathrm{C}(1.78 \mathrm{~g})$, and the mixture was stirred for $8 \mathrm{~h}$, while $\mathrm{H}_{2}$ was bubbled through. After filtration over hyflo and concentration, column chromatography $\left(19: 1 \mathrm{CH}_{2} \mathrm{Cl}_{2}-\mathrm{MeOH}\right)$ of the residue gave a colourless syrup $(0.94 \mathrm{~g}, 90 \%)$. A soln of the syrup in $\mathrm{Py}(75 \mathrm{~mL})$ and $\mathrm{Ac}_{2} \mathrm{O}(75 \mathrm{~mL})$ was stirred overnight, then co-concentrated with toluene, EtOH, and $\mathrm{CH}_{2} \mathrm{Cl}_{2}$. Low-pressure column chromatography (9:1 toluene-EtOAc) of the residue gave 26, isolated as a colourless syrup (1.08 g, quantitatively). To a soln of $26(0.11 \mathrm{~g}, 78 \mu \mathrm{mol})$ in dry DMF $(5 \mathrm{~mL})$ was added hydrazinium acetate $(9 \mathrm{mg}, 118 \mu \mathrm{mol})$. The mixture was stirred for $4 \mathrm{~h}$, washed with aq satd $\mathrm{NaCl}$, dried, filtered, and co-concentrated with toluene. Low-pressure column chromatography $(1: 1 \rightarrow 2: 3$ toluene-EtOAc) of the residue gave 27 , isolated as a white solid $(69 \mathrm{mg}, 65 \%) ; R_{f}$ 0.42 (1:1 toluene-EtOAc); ${ }^{1} \mathrm{H}$ NMR $\left(500 \mathrm{MHz}, \mathrm{CDCl}_{3}\right.$; 2D TOCSY, ROESY): $\delta 1.05$ [s, $9 \mathrm{H},\left(\mathrm{CH}_{3}\right)_{3} \mathrm{CSi}$ ], 1.88, $1.96,2.03,2.10$, and $2.14\left(5 \mathrm{~s}, 3,3,6,3,3 \mathrm{H}, 6 \mathrm{COCH}_{3}\right)$, 2.25 and $2.34\left(2 \mathrm{~s}\right.$, each $\left.3 \mathrm{H}, 2 \mathrm{CH}_{3} \mathrm{C}_{6} \mathrm{H}_{4} \mathrm{CO}\right), 3.48(\mathrm{~m}, 1$ $\left.\mathrm{H}, \mathrm{H}-5^{\mathrm{II}}\right), 3.54\left(\mathrm{~m}, 0.5 \mathrm{H}, \mathrm{H}-5^{\mathrm{I} \beta}\right), 3.83\left(\mathrm{dd}, 1 \mathrm{H}, J_{5,6 \mathrm{a}} 1.3\right.$, $\left.J_{6 \mathrm{a}, 6 \mathrm{~b}} 11.0 \mathrm{~Hz}, \mathrm{H}-6 \mathrm{a}^{\mathrm{III}}\right), 4.25\left(\mathrm{~d}, 1 \mathrm{H}, J_{1,2} 8.1 \mathrm{~Hz}, \mathrm{H}^{1{ }^{\mathrm{II}}}\right)$, $4.38\left(\mathrm{dd}, 1 \mathrm{H}, J_{1,2} 8.3, J_{2,3} 10.8 \mathrm{~Hz}, \mathrm{H}-2^{\mathrm{III}}\right), 4.66(\mathrm{~d}, 0.5$ $\left.\mathrm{H}, J_{1 \beta, 2 \beta} 7.9 \mathrm{~Hz}, \mathrm{H}-1^{\mathrm{I} \beta}\right), 4.76\left(\mathrm{dd}, 0.5 \mathrm{H}, J_{2 \beta, 3 \beta} 9.6 \mathrm{~Hz}\right.$, $\left.\mathrm{H}-2^{\mathrm{I} \beta}\right), 4.77\left(\mathrm{dd}, 0.5 \mathrm{H}, J_{1 \alpha, 2 \alpha} 3.5, J_{2 \alpha, 3 \alpha} 10.1 \mathrm{~Hz}, \mathrm{H}-2^{\mathrm{I} \alpha}\right)$, $4.84\left(\mathrm{dd}, 1 \mathrm{H}, J_{2,3} 9.8 \mathrm{~Hz}, \mathrm{H}-2^{\mathrm{II}}\right), 5.08\left(\mathrm{t}, 0.5 \mathrm{H}, \mathrm{H}-3^{\mathrm{I} \beta}\right)$, $5.31\left(\mathrm{~d}, 0.5 \mathrm{H}, \mathrm{H}-1^{\mathrm{I} \alpha}\right), 5.38\left(\mathrm{t}, 0.5 \mathrm{H}, \mathrm{H}-3^{\mathrm{I} \alpha}\right), 5.44(\mathrm{t}, 1 \mathrm{H}$, $\left.\mathrm{H}-4^{\mathrm{III}}\right), 6.20\left(\mathrm{~m}, 1 \mathrm{H}, \mathrm{H}-3^{\mathrm{III}}\right), 7.02$ and $7.12(2 \mathrm{~d}$, each 2 $\mathrm{H}$, Phth); HRMS of $\mathrm{C}_{70} \mathrm{H}_{77} \mathrm{NO}_{25} \mathrm{Si}(\mathrm{M}, 1359.455)$ : [M+ $\mathrm{H}]^{+}$found 1360.424 , calcd 1360.463 .

\subsection{0. (6-O-Tert-butyldiphenylsilyl-2-deoxy-3,4-di- $O$-p- methylbenzoyl-2-phthalimido- $\beta$-D-glucopyranosyl)-( $1 \rightarrow$ $3)-(2,4,6$-tri- $O$-acetyl- $\beta$-D-galactopyranosyl)-(1 $\rightarrow 4)$ - 2,3,6-tri- $O$-acetyl- $\alpha, \beta$-D-glucopyranosyl trichloroacetimidate (28)}

To a soln of $27(75 \mathrm{mg}, 55 \mu \mathrm{mol})$ in dry $\mathrm{CH}_{2} \mathrm{Cl}_{2}(0.5 \mathrm{~mL})$ were added, at $0{ }^{\circ} \mathrm{C}$ under Ar, trichloroacetonitrile (29 $\mu \mathrm{L}, 0.29 \mu \mathrm{mol})$ and 1,8-diazabicyclo[5.4.0]undec-7-ene $(0.90 \mu \mathrm{L})$. After stirring at $\mathrm{rt}$ for $1.5 \mathrm{~h}$, followed by concentration, low-pressure column chromatography $\left(1: 1\right.$ toluene-EtOAc with $\left.1 \% \mathrm{Et}_{3} \mathrm{~N}\right)$ of the residue gave $\mathbf{2 8}$, isolated as a slightly yellow foam (58 $\mathrm{mg}, 69 \%$ ); $R_{f}$ 0.63 (1:1 toluene-EtOAc); ${ }^{1} \mathrm{H}$ NMR $\alpha$-product $(300$ $\left.\mathrm{MHz}, \mathrm{CDCl}_{3}\right): \delta 1.06$ [s, $\left.9 \mathrm{H},\left(\mathrm{CH}_{3}\right)_{3} \mathrm{CSi}\right], 1.88,1.97$, $1.98,2.05,2.10$, and $2.15\left(6 \mathrm{~s}\right.$, each $\left.3 \mathrm{H}, 6 \mathrm{COCH}_{3}\right), 2.24$ and $2.34\left(2 \mathrm{~s}\right.$, each $\left.3 \mathrm{H}, 2 \mathrm{CH}_{3} \mathrm{C}_{6} \mathrm{H}_{4} \mathrm{CO}\right), 3.50(\mathrm{~m}, 1 \mathrm{H}$, $\left.\mathrm{H}-5^{\mathrm{II}}\right), 3.75\left(\mathrm{t}, 1 \mathrm{H}, \mathrm{H}-4^{\mathrm{I}}\right), 4.27\left(\mathrm{~d}, 1 \mathrm{H}, J_{1,2} 8.0 \mathrm{~Hz}, \mathrm{H}-\right.$ $\left.1^{\mathrm{II}}\right), 4.38\left(\mathrm{dd}, 1 \mathrm{H}, J_{1,2} 8.1, J_{2,3} 10.8 \mathrm{~Hz}, \mathrm{H}-2^{\mathrm{III}}\right), 4.44(\mathrm{dd}$, $\left.1 \mathrm{H}, J_{5,6 \mathrm{a}} 1.5, J_{6 \mathrm{a}, 6 \mathrm{~b}} 11.7 \mathrm{~Hz}, \mathrm{H}-6 \mathrm{a}\right), 4.85\left(\mathrm{dd}, 1 \mathrm{H}, J_{2,3}\right.$ $\left.9.8 \mathrm{~Hz}, \mathrm{H}-2^{\mathrm{II}}\right), 5.02\left(\mathrm{dd}, 1 \mathrm{H}, J_{1,2} 3.8, J_{2,3} 10.2 \mathrm{~Hz}, \mathrm{H}-2^{\mathrm{I}}\right)$, $6.24\left(\mathrm{dd}, 1 \mathrm{H}, J_{3,4} 9.2 \mathrm{~Hz}, \mathrm{H}-3^{\mathrm{III}}\right), 6.45\left(\mathrm{~d}, 1 \mathrm{H}, \mathrm{H}-1^{\mathrm{I}}\right)$, 7.01 and $7.11(2 \mathrm{~d}$, each $2 \mathrm{H}$, Phth), $8.63(\mathrm{~s}, 1 \mathrm{H}$, $\mathrm{OC}[\mathrm{NH}] \mathrm{CCl}_{3}$ ). 
3.21. 6-Azidohexyl (6-O-tert-butyldiphenylsilyl-2-deoxy3,4-di- $O$ - $p$-methylbenzoyl-2-phthalimido- $\beta$-Dglucopyranosyl)-( $1 \rightarrow 3)-(2,4,6$-tri- $O$-acetyl- $\beta$-Dgalactopyranosyl)-( $(\rightarrow 4)-(2,3,6$-tri- $O$-acetyl- $\beta$-Dglucopyranosyl)-(1 $\rightarrow 6)-2$-deoxy-3,4-di- $O-p$ methylbenzoyl-2-phthalimido- $\beta$-D-glucopyranoside (29)

A soln of $28(0.36 \mathrm{~g}, 0.24 \mathrm{mmol})$ and $11(0.18 \mathrm{~g}, 0.29$ mmol) in dry $\mathrm{CH}_{2} \mathrm{Cl}_{2}(5 \mathrm{~mL})$, containing molecular sieves $4 \AA$ ( $0.1 \mathrm{~g})$, was stirred for $30 \mathrm{~min}$ under Ar. The mixture was cooled to $-40{ }^{\circ} \mathrm{C}$, and TMSOTf $(4 \mu \mathrm{L}, 24$ $\mu \mathrm{mol})$ was added. The mixture was stirred for $2 \mathrm{~h}$, during which period the temperature was allowed to reach $\mathrm{rt}$, then neutralized with $\mathrm{Et}_{3} \mathrm{~N}$, washed with aq satd $\mathrm{NaHCO}_{3}$ and water, dried, filtered, and concentrated. Column chromatography (3:1 toluene-EtOAc) of the residue gave crude 29 after concentration. Gelfiltration of the residue on a LH-20 column eluted with $\mathrm{CH}_{2} \mathrm{Cl}_{2}-\mathrm{MeOH}$ (1:1) gave 29, isolated as a white solid $(0.18 \mathrm{~g}, 38 \%) ; R_{f} 0.88\left(1: 1\right.$ toluene-EtOAc); $[\alpha]_{\mathrm{D}}^{20}+4^{\circ}(c$ $\left.1, \mathrm{CHCl}_{3}\right) ;{ }^{1} \mathrm{H}$ NMR $\left(500 \mathrm{MHz}, \mathrm{CDCl}_{3} ; 2 \mathrm{D}\right.$ TOCSY, ROESY): $\delta 1.05\left[\mathrm{~s}, 9 \mathrm{H},\left(\mathrm{CH}_{3}\right)_{3} \mathrm{CSi}\right], 1.11-1.16(\mathrm{~m}, 4 \mathrm{H}$, $\left.2 \mathrm{CH}_{2}\right), 1.25-1.29\left(\mathrm{~m}, 2 \mathrm{H}, \mathrm{CH}_{2}\right), 1.42-1.50(\mathrm{~m}, 2 \mathrm{H}$, $\left.\mathrm{CH}_{2}\right), 1.88,1.94,2.02,2.03,2.04$, and $2.12(6 \mathrm{~s}$, each $3 \mathrm{H}$, $\left.6 \mathrm{COCH}_{3}\right), 2.25,2.26$, and $2.33(3 \mathrm{~s}, 3,3,6 \mathrm{H}, 4$ $\left.\mathrm{CH}_{3} \mathrm{C}_{6} \mathrm{H}_{4} \mathrm{CO}\right), 3.02\left(\mathrm{t}, 2 \mathrm{H}, \mathrm{CH}_{2} \mathrm{~N}_{3}\right), 3.62(\mathrm{t}, 1 \mathrm{H}, \mathrm{H}-$ $\left.4^{\mathrm{II}}\right), 3.76\left(\mathrm{dd}, 1 \mathrm{H}, J_{5,6 \mathrm{~b}} 7.7, J_{6 \mathrm{a}, 6 \mathrm{~b}} 11.6 \mathrm{~Hz}, \mathrm{H}-6 \mathrm{~b}^{\mathrm{IV}}\right), 3.83$ $\left(\mathrm{dd}, 1 \mathrm{H}, J_{5,6 \mathrm{a}} 1.5, J_{6 \mathrm{a}, 6 \mathrm{~b}} 11.2 \mathrm{~Hz}, \mathrm{H}-6 \mathrm{a}^{\mathrm{I}}\right), 4.18(\mathrm{~d}, 1 \mathrm{H}$, $\left.J_{1,2} 8.1 \mathrm{~Hz}, \mathrm{H}-1^{\mathrm{III}}\right), 4.46\left(\mathrm{dd}, 1 \mathrm{H}, J_{1,2} 8.5, J_{2,3} 10.8 \mathrm{~Hz}\right.$, $\left.\mathrm{H}-2^{\mathrm{IV}}\right), 4.57\left(\mathrm{~d}, 1 \mathrm{H}, J_{1,2} 7.9 \mathrm{~Hz}, \mathrm{H}-1^{\mathrm{II}}\right), 4.81(\mathrm{dd}, 1 \mathrm{H}$, $\left.J_{2,3} 9.8 \mathrm{~Hz}, \mathrm{H}-2^{\mathrm{III}}\right), 4.87\left(\mathrm{dd}, 1 \mathrm{H}, J_{2,3} 9.0 \mathrm{~Hz}, \mathrm{H}-2^{\mathrm{II}}\right)$, $5.01\left(\mathrm{t}, 1 \mathrm{H}, \mathrm{H}-3^{\mathrm{II}}\right), 5.33\left(\mathrm{t}, 1 \mathrm{H}, \mathrm{H}-4^{\mathrm{IV}}\right), 5.47\left(\mathrm{~d}, 1 \mathrm{H}, J_{1,2}\right.$ $\left.8.1 \mathrm{~Hz}, \mathrm{H}-1^{\mathrm{I}}\right) ;{ }^{13} \mathrm{C}$ NMR $\left(75.5 \mathrm{MHz}, \mathrm{CDCl}_{3}\right): \delta 19.1$ $\left[\left(\mathrm{CH}_{3}\right)_{3} \mathrm{CSi}\right], 20.4-20.6\left(\mathrm{COCH}_{3}\right), 21.4$ and 21.5 $\left(\mathrm{CH}_{3} \mathrm{C}_{6} \mathrm{H}_{4} \mathrm{CO}\right), 25.3,26.1,28.4$, and $29.0\left(4 \mathrm{CH}_{2}\right)$, $26.6\left[\left(\mathrm{CH}_{3}\right)_{3} \mathrm{CSi}\right], 51.0\left(\mathrm{CH}_{2} \mathrm{~N}_{3}\right), 54.8$ and $55.0\left(\mathrm{C}-2^{1}\right.$, $\left.\mathrm{C}-2^{\mathrm{IV}}\right), 61.3,62.1,63.0,68.3$, and $69.8\left(\mathrm{C}-6^{\mathrm{I}}, \mathrm{C}-6^{\mathrm{II}}, \mathrm{C}-\right.$ $\left.6^{\mathrm{III}}, \mathrm{C}-6^{\mathrm{IV}}, \mathrm{OCH}_{2}\right), 68.8,69.8,69.9,70.3,70.8,70.9$, 71.4, 72.4, 72.7, 74.1, 74.4 (2 C), 75.3, and 75.6 (C-3 ${ }^{\mathrm{I}}, \mathrm{C}-$ $4^{\mathrm{I}}, \mathrm{C}-5^{\mathrm{I}}, \mathrm{C}-2^{\mathrm{II}}, \mathrm{C}-3^{\mathrm{II}}, \mathrm{C}-4^{\mathrm{II}}, \mathrm{C}-5^{\mathrm{II}}, \mathrm{C}-2^{\mathrm{III}}, \mathrm{C}-3^{\mathrm{III}}, \mathrm{C}-4^{\mathrm{III}}$, $\left.\mathrm{C}-5^{\mathrm{III}}, \mathrm{C}-3^{\mathrm{IV}}, \mathrm{C}-4^{\mathrm{IV}}, \mathrm{C}-5^{\mathrm{IV}}\right), 97.9,98.0,100.5$, and 100.6 $\left(\mathrm{C}-1^{\mathrm{I}}, \mathrm{C}-1^{\mathrm{II}}, \mathrm{C}-1^{\mathrm{III}}, \mathrm{C}-1^{\mathrm{IV}}\right), 164.9,165.2,165.4$, and 165.5 (4 $\left.\mathrm{CH}_{3} \mathrm{C}_{6} \mathrm{H}_{4} \mathrm{CO}\right), 168.7,169.3,169.4,169.7,170.1$, and $170.2\left(6 \mathrm{COCH}_{3}\right)$; HRMS of $\mathrm{C}_{106} \mathrm{H}_{113} \mathrm{~N}_{5} \mathrm{O}_{33} \mathrm{Si}(\mathrm{M}$, 2011.708): $[\mathrm{M}+\mathrm{H}]^{+}$found 2012.795, calcd 2012.717.

3.22. 6-Azidohexyl (2-deoxy-3,4-di- $O$-p -methylbenzoyl2-phthalimido- $\beta$-D-glucopyranosyl)-( $1 \rightarrow 3)-(2,4,6$-tri- $O$ acetyl- $\beta$-D-galactopyranosyl)-(1 $\rightarrow 4)-(2,3,6$-tri- $O$-acetyl$\beta$-D-glucopyranosyl)-( $\rightarrow$ 6)-2-deoxy-3,4-di- $O$ - $p$ methylbenzoyl-2-phthalimido- $\beta$-D-glucopyranoside (30)

A soln of $29(60 \mathrm{mg}, 29.8 \mu \mathrm{mol})$ in $1.0 \mathrm{M}$ TBAF in THF $(0.7 \mathrm{~mL})$ and $\mathrm{AcOH}(0.7 \mathrm{~mL})$ was stirred for $1 \mathrm{~h}$ at $\mathrm{pH} 6$, at $0{ }^{\circ} \mathrm{C}$, then for $3 \mathrm{~h}$ at $\mathrm{rt}$. The mixture was washed with water and aq $10 \% \mathrm{NaCl}$, dried, filtered, and concen- trated. Column chromatography (1:1 toluene-EtOAc) of the residue gave 30, isolated as a white solid $(48 \mathrm{mg}$, $90 \%) ; R_{f} 0.42\left(1: 1\right.$ toluene-EtOAc); $[\alpha]_{\mathrm{D}}^{20}+2^{\circ}(c \quad 1$, $\left.\mathrm{CHCl}_{3}\right) ;{ }^{1} \mathrm{H}$ NMR $\left(500 \mathrm{MHz}, \mathrm{CDCl}_{3} ; 2 \mathrm{D}\right.$ TOCSY, ROESY): $\delta 1.10-1.16\left(\mathrm{~m}, 4 \mathrm{H}, 2 \mathrm{CH}_{2}\right), 1.22-1.29(\mathrm{~m}, 2$ $\left.\mathrm{H}, \mathrm{CH}_{2}\right), 1.42-1.46\left(\mathrm{~m}, 2 \mathrm{H}, \mathrm{CH}_{2}\right), 1.96,1.97,2.01,2.04$, 2.07, and $2.21\left(6 \mathrm{~s}\right.$, each $\left.3 \mathrm{H}, 6 \mathrm{COCH}_{3}\right), 2.27$ and $2.33(2$ s, each $\left.6 \mathrm{H}, 4 \mathrm{CH}_{3} \mathrm{C}_{6} \mathrm{H}_{4} \mathrm{CO}\right), 3.03\left(\mathrm{t}, 2 \mathrm{H}, \mathrm{CH}_{2} \mathrm{~N}_{3}\right), 3.96$ $\left(\mathrm{dd}, 1 \mathrm{H}, J_{5,6 \mathrm{~b}} 5.7, J_{6 \mathrm{a}, 6 \mathrm{~b}} 11.9 \mathrm{~Hz}, \mathrm{H}-6 \mathrm{~b}^{\mathrm{II}}\right), 4.27(\mathrm{dd}, 1 \mathrm{H}$, $\left.J_{5,6 \mathrm{a}} 1.9 \mathrm{~Hz}, \mathrm{H}-6 \mathrm{a}^{\mathrm{II}}\right), 4.28\left(\mathrm{~d}, 1 \mathrm{H}, J_{1,2} 8.1 \mathrm{~Hz}, \mathrm{H}-1^{\mathrm{III}}\right)$, $4.39\left(\mathrm{dd}, 1 \mathrm{H}, J_{1,2} 8.3, J_{2,3} 10.9 \mathrm{~Hz}, \mathrm{H}-2^{\mathrm{IV}}\right), 4.44(\mathrm{dd}, 1$ $\left.\mathrm{H}, J_{1,2} 8.5, J_{2,3} 10.8 \mathrm{~Hz}, \mathrm{H}-2^{\mathrm{I}}\right), 4.57\left(\mathrm{~d}, 1 \mathrm{H}, J_{1,2} 7.9 \mathrm{~Hz}\right.$, $\left.\mathrm{H}-1^{\mathrm{II}}\right), 4.86\left(\mathrm{dd}, 1 \mathrm{H}, J_{2,3} 9.4 \mathrm{~Hz}, \mathrm{H}-2^{\mathrm{II}}\right), 4.90(\mathrm{dd}, 1 \mathrm{H}$, $\left.J_{2,3} 9.9 \mathrm{~Hz}, \mathrm{H}-2^{\mathrm{III}}\right), 5.04\left(\mathrm{t}, 1 \mathrm{H}, \mathrm{H}-3^{\mathrm{II}}\right), 5.31(\mathrm{dd}, 1 \mathrm{H}$, $\left.J_{3,4} 9.0, J_{4,5} 10.1 \mathrm{~Hz}, \mathrm{H}-4^{\mathrm{I}}\right), 5.43\left(\mathrm{~d}, 1 \mathrm{H}, \mathrm{H}-1^{\mathrm{I}}\right), 5.46(\mathrm{t}, 1$ $\left.\mathrm{H}, \mathrm{H}-4^{\mathrm{IV}}\right), 5.56\left(\mathrm{~d}, 1 \mathrm{H}, J_{3,4} 3.9, J_{4,5}<1 \mathrm{~Hz}, \mathrm{H}-4^{\mathrm{III}}\right), 5.60$ $\left(\mathrm{d}, 1 \mathrm{H}, \mathrm{H}-1^{\mathrm{IV}}\right), 6.12\left(\mathrm{dd}, 1 \mathrm{H}, J_{3,4} 9.2 \mathrm{~Hz}, \mathrm{H}-3^{\mathrm{IV}}\right), 6.16$ $\left(\mathrm{dd}, 1 \mathrm{H}, \mathrm{H}-3{ }^{\mathrm{I}}\right) ;{ }^{13} \mathrm{C} \mathrm{NMR}\left(75.5 \mathrm{MHz}, \mathrm{CDCl}_{3}\right): \delta 19.5-$ $20.8\left(\mathrm{COCH}_{3}\right), 21.4\left(\mathrm{CH}_{3} \mathrm{C}_{6} \mathrm{H}_{4} \mathrm{CO}\right), 25.3,26.1,28.4$, and $28.9\left(4 \mathrm{CH}_{2}\right), 51.0\left(\mathrm{CH}_{2} \mathrm{~N}_{3}\right), 54.6$ and $54.8\left(\mathrm{C}-2^{\mathrm{I}}\right.$, C$\left.2^{\mathrm{IV}}\right), 61.2,62.0,68.2$, and $69.6(2 \mathrm{C})\left(\mathrm{C}-6^{\mathrm{I}}, \mathrm{C}-6^{\mathrm{II}}, \mathrm{C}-6^{\mathrm{III}}\right.$, $\left.\mathrm{C}-6^{\mathrm{IV}}, \mathrm{OCH}_{2}\right), 68.9,69.0,69.9,70.2,70.3,70.8(2 \mathrm{C})$, 71.4, 72.5 (2 C), 74.1, 74.9, 75.6, and 78.2 (C-3 ${ }^{\mathrm{I}}, \mathrm{C}-4^{\mathrm{I}}, \mathrm{C}-$ $5^{\mathrm{I}}, \mathrm{C}-2^{\mathrm{II}}, \mathrm{C}-3^{\mathrm{II}}, \mathrm{C}-4^{\mathrm{II}}, \mathrm{C}-5^{\mathrm{II}}, \mathrm{C}-2^{\mathrm{III}}, \mathrm{C}-3^{\mathrm{III}}, \mathrm{C}-4^{\mathrm{III}}, \mathrm{C}-5^{\mathrm{III}}$, $\left.\mathrm{C}-3^{\mathrm{IV}}, \mathrm{C}-4^{\mathrm{IV}}, \mathrm{C}-5^{\mathrm{IV}}\right), 98.0,98.8,100.4$, and $100.5\left(\mathrm{C}-1^{\mathrm{I}}\right.$, $\left.\mathrm{C}-1^{\mathrm{II}}, \mathrm{C}-1^{\mathrm{III}}, \mathrm{C}-1^{\mathrm{IV}}\right), 165.1$ and $165.4\left(\mathrm{CH}_{3} \mathrm{C}_{6} \mathrm{H}_{4} \mathrm{CO}\right)$, 168.1-170.9 $\left(\mathrm{COCH}_{3}\right)$; HRMS of $\mathrm{C}_{90} \mathrm{H}_{95} \mathrm{~N}_{5} \mathrm{O}_{33}(\mathrm{M}$, 1773.591): $[\mathrm{M}+\mathrm{H}]^{+}$found 1774.675, calcd 1774.599.

\subsection{6-Azidohexyl $(2,3,4,6$-tetra- $O$-acetyl- $\beta$-D- galactopyranosyl)-( $(1 \rightarrow 4)-(2,3,6$-tri- $O$-acetyl- $\beta$-D- glucopyranosyl)-(1 $\rightarrow 6)-(2-$ deoxy-3,4-di- $O$ - $p$ - methylbenzoyl-2-phthalimido- $\beta$-D-glucopyranosyl)-(1 $\rightarrow$ $3)-(2,4,6$-tri- $O$-acetyl- $\beta$-D-galactopyranosyl)- $(1 \rightarrow 4)$ - $(2,3,6$-tri- $O$-acetyl- $\beta$-D-glucopyranosyl)-( $(\rightarrow 6)-2$-deoxy- 3,4-di- $O$ - $p$-methylbenzoyl-2-phthalimido- $\beta$-D- glucopyranoside (31)}

(a) A soln of $19(129 \mathrm{mg}, 103 \mu \mathrm{mol})$ and (2,3,4,6-tetra$O$-acetyl- $\beta$-D-galactopyranosyl)-( $1 \rightarrow 4)-(2,3,6$-tri- $O$-acetyl- $\beta$-D-glucopyranosyl)-( $(1 \rightarrow 6)-2$-deoxy-3,4-di- $O$ - $p$ methylbenzoyl-2-phthalimido- $\beta$-D-glucopyranosyl trichloroacetimidate $(\mathbf{2 0})^{17}(202 \mathrm{mg}, 155 \mu \mathrm{mol})$ in dry $\mathrm{CH}_{2} \mathrm{Cl}_{2}(2 \mathrm{~mL})$, containing powdered molecular sieves 4 $\AA(0.2 \mathrm{~g})$, was stirred for $30 \mathrm{~min}$ under $\mathrm{Ar}$, then cooled to $-20^{\circ} \mathrm{C}$, and TMSOTf $(3 \mu \mathrm{L}, 16 \mu \mathrm{mol})$ was added. The mixture was stirred for $1 \mathrm{~h}$, during which period the temperature was allowed to reach $\mathrm{rt}$, then neutralized with $\mathrm{Et}_{3} \mathrm{~N}$, filtered over hyflo, and concentrated. Column chromatography (1:2 toluene-EtOAc) of the residue yielded after concentration crude 31. Gel-filtration of the residue on a LH-20 column, eluted with $\mathrm{CH}_{2} \mathrm{Cl}_{2}-\mathrm{MeOH}$ (1:1), gave 31, isolated as white foam (30 mg, 12\%).

(b) A soln of $30(84 \mathrm{mg}, 47 \mu \mathrm{mol})$ and $(\mathbf{1 2})^{19}(125 \mathrm{mg}$, $160 \mu \mathrm{mol})$ in dry $\mathrm{CH}_{2} \mathrm{Cl}_{2}(1 \mathrm{~mL})$, containing powdered 
molecular sieves $4 \AA(0.1 \mathrm{~g})$, was stirred for $0.5 \mathrm{~h}$ under Ar, then cooled to $-40{ }^{\circ} \mathrm{C}$, and TMSOTf $(3 \mu \mathrm{L}, 16$ $\mu \mathrm{mol})$ was added. The mixture was stirred for $30 \mathrm{~min}$ at $-40{ }^{\circ} \mathrm{C}$, then at $\mathrm{rt}$ for $3 \mathrm{~h}$, and concentrated. Column chromatography (1:1 toluene-EtOAc) of the residue yielded crude 31. Gel-filtration of the residue on a LH20 column, eluted with $1: 1 \mathrm{CH}_{2} \mathrm{Cl}_{2}-\mathrm{MeOH}$, gave 31, isolated as a white foam $(68 \mathrm{mg}, 60 \%) ; R_{f} 0.74(1: 2$ toluene-EtOAc); $[\alpha]_{\mathrm{D}}^{20}-4^{\circ}\left(c 1, \mathrm{CHCl}_{3}\right) ;{ }^{1} \mathrm{H}$ NMR $(500$ $\mathrm{MHz}, \mathrm{CDCl}_{3}$; 2D TOCSY, ROESY, HSQC): $\delta 1.08-$ $1.17\left(\mathrm{~m}, 4 \mathrm{H}, 2 \mathrm{CH}_{2}\right), 1.23-1.30\left(\mathrm{~m}, 2 \mathrm{H}, \mathrm{CH}_{2}\right), 1.41-$ $1.49\left(\mathrm{~m}, 2 \mathrm{H}, \mathrm{CH}_{2}\right), 1.96,2.01,2.03,2.05,2.06,2.07$, 2.12, 2.15, and $2.16\left(9 \mathrm{~s}, 9,3,6,3,3,6,3,3,3 \mathrm{H}, 13 \mathrm{COCH}_{3}\right)$, 2.25, 2.27, 2.33, and $2.34\left(4 \mathrm{~s}\right.$, each $\left.3 \mathrm{H}, 4 \mathrm{CH}_{3} \mathrm{C}_{6} \mathrm{H}_{4} \mathrm{CO}\right)$, $3.02\left(\mathrm{t}, 2 \mathrm{H}, \mathrm{CH}_{2} \mathrm{~N}_{3}\right), 3.75\left(\mathrm{dd}, 1 \mathrm{H}, J_{5,6 \mathrm{a}} 7.9, J_{6 \mathrm{a}, 6 \mathrm{~b}} 11.8\right.$ $\left.\mathrm{Hz}, \mathrm{H}-6 \mathrm{a}^{\mathrm{I}}\right), 3.80\left(\mathrm{t}, 1 \mathrm{H}, \mathrm{H}-4^{\mathrm{V}}\right), 4.45\left(\mathrm{dd}, 1 \mathrm{H}, J_{1,2} 8.5\right.$, $\left.J_{2,3} 10.7 \mathrm{~Hz}, \mathrm{H}-2^{\mathrm{I}}\right), 4.52\left(\mathrm{~d}, 1 \mathrm{H}, J_{1,2} 7.9 \mathrm{~Hz}, \mathrm{H}-1^{\mathrm{VI}}\right), 4.57$ $\left(\mathrm{d}, 1 \mathrm{H}, J_{1,2} 7.9 \mathrm{~Hz}, \mathrm{H}-1^{\mathrm{II}}\right), 4.63\left(\mathrm{~d}, 1 \mathrm{H}, J_{1,2} 7.7 \mathrm{~Hz}, \mathrm{H}-\right.$ $\left.1^{\mathrm{v}}\right), 4.95\left(\mathrm{dd}, 1 \mathrm{H}, J_{2,3} 10.5, J_{3,4} 3.3 \mathrm{~Hz}, \mathrm{H}-3^{\mathrm{VI}}\right), 5.00(\mathrm{t}, 1$ $\left.\mathrm{H}, J_{2,3} 9.6, J_{3,4} 9.2 \mathrm{~Hz}, \mathrm{H}-3^{\mathrm{II}}\right), 5.41\left(\mathrm{~d}, 1 \mathrm{H}, J_{3,4} 3.7\right.$, $\left.J_{4,5}<1 \mathrm{~Hz}, \mathrm{H}-4^{\mathrm{III}}\right), 5.43\left(\mathrm{~d}, 1 \mathrm{H}, \mathrm{H}-1^{\mathrm{I}}\right), 5.44\left(\mathrm{~d}, 1 \mathrm{H}, J_{1,2}\right.$ $\left.8.1 \mathrm{~Hz}, \mathrm{H}-1{ }^{\mathrm{VI}}\right) ;{ }^{13} \mathrm{C}$ NMR $\left(75.5 \mathrm{MHz}, \mathrm{CDCl}_{3}\right): \delta 20.4-$ $20.6\left(\mathrm{COCH}_{3}\right), 21.4$ and $21.5\left(\mathrm{CH}_{3} \mathrm{C}_{6} \mathrm{H}_{4} \mathrm{CO}\right), 25.3,26.1$, 28.4, and $28.9\left(4 \mathrm{CH}_{2}\right), 51.0\left(\mathrm{CH}_{2} \mathrm{~N}_{3}\right), 54.8(2 \mathrm{C})\left(\mathrm{C}-2^{\mathrm{I}}\right.$, $\left.\mathrm{C}-2^{\mathrm{IV}}\right), 60.4\left(\mathrm{C}-6^{\mathrm{III}}\right), 61.6\left(\mathrm{C}-6^{\mathrm{VI}}\right), 62.2\left(\mathrm{C}-6^{\mathrm{V}}\right), 62.4(\mathrm{C}-$ $\left.6^{\mathrm{II}}\right), 68.3\left(\mathrm{C}-6^{\mathrm{I}}\right), 68.7\left(\mathrm{C}-6^{\mathrm{IV}}\right), 69.6\left(\mathrm{OCH}_{2}\right), 66.4\left(\mathrm{C}-4^{\mathrm{VI}}\right)$, 68.9 (2 C) $\left(\mathrm{C}-4^{\mathrm{III}}, \mathrm{C}-2^{\mathrm{VI}}\right), 69.8(2 \mathrm{C})\left(\mathrm{C}-4^{\mathrm{I}}, \mathrm{C}-4^{\mathrm{IV}}\right), 69.9$ $\left(\mathrm{C}-3^{\mathrm{IV}}\right), 70.5\left(\mathrm{C}-5^{\mathrm{VI}}\right), 70.7\left(\mathrm{C}-3^{\mathrm{I}}\right), 70.8(2 \mathrm{C})\left(\mathrm{C}-3^{\mathrm{III}}, \mathrm{C}-\right.$ $\left.3^{\mathrm{VI}}\right), 71.0\left(\mathrm{C}-2^{\mathrm{III}}\right), 71.3\left(\mathrm{C}-2^{\mathrm{II}}\right), 71.8\left(\mathrm{C}-2^{\mathrm{V}}\right), 72.3\left(\mathrm{C}-3^{\mathrm{II}}\right)$, $72.7\left(\mathrm{C}-5^{\mathrm{V}}\right), 72.8\left(\mathrm{C}-5^{\mathrm{II}}\right), 72.9\left(\mathrm{C}-3^{\mathrm{V}}\right), 74.1(2 \mathrm{C})$ and 74.9 $\left(\mathrm{C}-5^{\mathrm{I}}, \mathrm{C}-5^{\mathrm{III}}, \mathrm{C}-5^{\mathrm{IV}}\right), 75.7\left(\mathrm{C}-4^{\mathrm{II}}\right), 76.1\left(\mathrm{C}-4^{\mathrm{V}}\right), 97.6$ and $98.0\left(\mathrm{C}-1^{\mathrm{I}}, \mathrm{C}-1^{\mathrm{IV}}\right), 100.3\left(\mathrm{C}-1^{\mathrm{III}}\right), 100.5\left(\mathrm{C}-1^{\mathrm{II}}\right), 100.8(\mathrm{C}-$ $\left.1^{\mathrm{V}}\right), 100.9\left(\mathrm{C}-1^{\mathrm{VI}}\right), 165.1,165.2,165.3$, and $165.4(4$ $\left.\mathrm{CH}_{3} \mathrm{C}_{6} \mathrm{H}_{4} \mathrm{CO}\right), \quad 168.6-170.9 \quad\left(\mathrm{COCH}_{3}\right) ;$ HRMS of $\mathrm{C}_{116} \mathrm{H}_{129} \mathrm{~N}_{5} \mathrm{O}_{50} \quad(\mathrm{M}, \quad 2391.770):[\mathrm{M}+\mathrm{H}]^{+}$found 2392.742, calcd 2392.778 .

\subsection{6-Azidohexyl $(2,3,4,6$-tetra- $O$-acetyl- $\beta$-D-} galactopyranosyl)-( $1 \rightarrow 4)-(2,3,6$-tri- $O$-acetyl- $\beta$-Dglucopyranosyl)-( $1 \rightarrow 6)$-(2-acetamido-3,4-di- $O$-acetyl-2deoxy- $\beta$-D-glucopyranosyl)-( $(1 \rightarrow 3)-(2,4,6$-tri- $O$-acetyl- $\beta$ D-galactopyranosyl)-( $1 \rightarrow 4)-(2,3,6$-tri- $O$-acetyl- $\beta$-Dglucopyranosyl)-( $1 \rightarrow 6)$-2-acetamido-3,4-di- $O$-acetyl-2deoxy- $\beta$-D-glucopyranoside (32)

To a soln of $31(30 \mathrm{mg}, 12.5 \mu \mathrm{mol})$ in $\mathrm{MeOH}(2 \mathrm{~mL})$ and $\mathrm{CH}_{2} \mathrm{Cl}_{2}(0.3 \mathrm{~mL})$ was added $\mathrm{NaOMe}(\mathrm{pH} 10)$, and the mixture was stirred for $24 \mathrm{~h}$. After neutralization with Dowex $50 \times 8\left(\mathrm{H}^{+}\right)$and filtration, the mixture was concentrated. To a solution of the residue in 1-BuOH (5 $\mathrm{mL}$ ) was added 1,2-diaminoethane $(1 \mathrm{~mL})$, and the mixture was stirred overnight at $80^{\circ} \mathrm{C}$, then, co-concentrated with toluene. A solution of the residue in Py $(10 \mathrm{~mL})$ and $\mathrm{Ac}_{2} \mathrm{O}(10 \mathrm{~mL})$ was stirred overnight, then co-concentrated with toluene. Column chromatography (9:1 $\left.\mathrm{CH}_{2} \mathrm{Cl}_{2}-\mathrm{MeOH}\right)$ of the residue afforded 32, iso- lated as a colourless glass (17 mg, 71\%); $R_{f} 0.30(9: 1$ $\left.\mathrm{CH}_{2} \mathrm{Cl}_{2}-\mathrm{MeOH}\right) ;[\alpha]_{\mathrm{D}}^{20}-2^{\circ}\left(c 1, \mathrm{CHCl}_{3}\right) ;{ }^{1} \mathrm{H}$ NMR (500 MHz, $\mathrm{CDCl}_{3}$; 2D TOCSY, ROESY): $\delta$ 1.37-1.38 (m, $4 \mathrm{H}, 2 \mathrm{CH}_{2}$ ), 1.58-1.61 (m, $\left.4 \mathrm{H}, 2 \mathrm{CH}_{2}\right), 1.90,1.93$, $1.96,1.98,1.99,2.01,2.02,2.03,2.06,2.07,2.08,2.09$, $2.10, \quad 2.12, \quad 2.13, \quad 2.14 \mathrm{~s}$ and $2.16 \quad(17 \mathrm{~s}$, $3,3,3,3,3,6,3,3,3,6,3,3,3,3,3,3,3 \quad \mathrm{H}, 2 \mathrm{NHCOCH}_{3}, 17$ $\left.\mathrm{COCH}_{3}\right), 3.26\left(\mathrm{t}, 2 \mathrm{H}, \mathrm{CH}_{2} \mathrm{~N}_{3}\right), 3.31\left(\mathrm{dd}, 1 \mathrm{H}, J_{1,2} 8.2\right.$, $\left.J_{2,3} 10.6 \mathrm{~Hz}, \mathrm{H}-2^{\mathrm{IV}}\right), 3.44(\mathrm{~m}, 1 \mathrm{H}, \mathrm{OCHH}), 3.97(\mathrm{dd}, 1$ $\left.\mathrm{H}, J_{2,3} 9.8, J_{3,4} 3.8 \mathrm{~Hz}, \mathrm{H}-3^{\mathrm{III}}\right), 4.53\left(\mathrm{~d}, 1 \mathrm{H}, J_{1,2} 7.9 \mathrm{~Hz}\right.$, $\left.\mathrm{H}-1^{\mathrm{IV}}\right), 4.56\left(\mathrm{~d}, 1 \mathrm{H}, J_{1,2} 8.0 \mathrm{~Hz}, \mathrm{H}-1^{\mathrm{II}}\right), 4.57\left(\mathrm{~d}, 1 \mathrm{H}, J_{1,2}\right.$ $\left.7.6 \mathrm{~Hz}, \mathrm{H}-1^{\mathrm{V}}\right), 4.60\left(\mathrm{~d}, 1 \mathrm{H}, J_{1,2} 8.3 \mathrm{~Hz}, \mathrm{H}-1^{\mathrm{I}}\right), 4.97(\mathrm{dd}, 1$ $\left.\mathrm{H}, J_{2,3} 10.4, J_{3,4} 3.4 \mathrm{~Hz}, \mathrm{H}-3^{\mathrm{IV}}\right), 5.02\left(\mathrm{dd}, 1 \mathrm{H}, J_{1,2} 8.0\right.$ $\left.\mathrm{Hz}, \mathrm{H}-2^{\mathrm{III}}\right), 5.08\left(\mathrm{dd}, 1 \mathrm{H}, \mathrm{H}-2^{\mathrm{IV}}\right), 5.12\left(\mathrm{t}, 1 \mathrm{H}, J_{2,3} 9.3\right.$, $\left.J_{3,4} 9.3 \mathrm{~Hz}, \mathrm{H}-3^{\mathrm{II}}\right), 5.12\left(\mathrm{dd}, 1 \mathrm{H}, J_{2,3} 9.4, J_{3,4} 9.5 \mathrm{~Hz}, \mathrm{H}-\right.$ $\left.3^{\mathrm{V}}\right), 5.25\left(\mathrm{dd}, 1 \mathrm{H}, J_{2,3} 10.6, J_{3,4} 9.3 \mathrm{~Hz}, \mathrm{H}-3^{\mathrm{I}}\right), 5.40(\mathrm{~d}, 1$ $\left.\mathrm{H}, J_{2, \mathrm{NH}} 7.8 \mathrm{~Hz}, \mathrm{NHCOCH} \mathrm{H}_{3}^{\mathrm{IV}}\right), 5.40\left(\mathrm{dd}, 1 \mathrm{H}, J_{3,4} 9.1\right.$ $\left.\mathrm{Hz}, \mathrm{H}-3^{\mathrm{IV}}\right), 5.45\left(\mathrm{~d}, 1 \mathrm{H}, J_{2, \mathrm{NH}} 8.7 \mathrm{~Hz}, \mathrm{~N} H \mathrm{COCH}_{3}^{\mathrm{I}}\right)$; HRMS of $\mathrm{C}_{80} \mathrm{H}_{113} \mathrm{~N}_{5} \mathrm{O}_{48}(\mathrm{M}, 1911.655):[\mathrm{M}+\mathrm{H}]^{+}$ found 1912.644, calcd 1912.663.

\subsection{6-Azidohexyl $\beta$-D-galactopyranosyl-( $1 \rightarrow 4)-\beta$-D- glucopyranosyl-( $1 \rightarrow 6)$-2-acetamido-2-deoxy- $\beta$-D- glucopyranosyl-( $(\rightarrow 3)-\beta$-D-galactopyranosyl- $(1 \rightarrow 4)-\beta$-D- glucopyranosyl-( $1 \rightarrow 6)$-2-acetamido-2-deoxy- $\beta$-D- glucopyranoside (33)}

To a soln of $32(17 \mathrm{mg}, 8.88 \mu \mathrm{mol})$ in $\mathrm{MeOH}(0.7 \mathrm{~mL})$ was added $\mathrm{NaOMe}(\mathrm{pH} 10)$. The mixture was stirred for $4 \mathrm{~h}$, then neutralized with Dowex $50 \times 8\left(\mathrm{H}^{+}\right)$, filtered, and concentrated. Gel-filtration of the residue on a Bio Gel P-2 column, eluted with aq $0.1 \mathrm{M} \mathrm{NH}_{4} \mathrm{HCO}_{3}$ at a flow rate of $40 \mathrm{~mL} / \mathrm{h}$, gave $\mathbf{3 3}$ after freeze-drying as a white powder $(9.4 \mathrm{mg}, 89 \%) ; R_{f} 0.19\left(1: 3 \mathrm{CH}_{2} \mathrm{Cl}_{2}-\right.$ $\mathrm{MeOH}) ; \quad[\alpha]_{\mathrm{D}}^{20}-1^{\circ} \quad(c \quad 0.5$, water); HRMS of $\mathrm{C}_{46} \mathrm{H}_{79} \mathrm{~N}_{5} \mathrm{O}_{31} \quad(\mathrm{M}, \quad 1197.475): \quad[\mathrm{M}+\mathrm{H}]^{+}$found 1198.508, calcd 1198.483. For ${ }^{1} \mathrm{H}$ NMR data, see Table 4.

\subsection{6-Aminohexyl $\beta$-D-galactopyranosyl-( $1 \rightarrow 4)-\beta$-D- glucopyranosyl- $(1 \rightarrow 6)$-2-acetamido-2-deoxy- $\beta$-D- glucopyranosyl-( $(\rightarrow 3)-\beta$-D-galactopyranosyl-( $(\rightarrow 4)-\beta$-D- glucopyranosyl-( $1 \rightarrow 6)$-2-acetamido-2-deoxy- $\beta$-D- glucopyranoside (4)}

To a soln of $33(8 \mathrm{mg}, 6.67 \mu \mathrm{mol})$ in $t$ - $\mathrm{BuOH}(3 \mathrm{~mL})$ and water $(2 \mathrm{~mL})$ were added $10 \% \mathrm{Pd}-\mathrm{C}(30 \mathrm{mg})$ and 2 drops of aq $25 \% \mathrm{NH}_{3}$, and the mixture was stirred for 24 $h$ under $\mathrm{H}_{2}$, then filtered over cotton, and concentrated. Gel-filtration of the residue on a Bio Gel P-2 column, eluted with aq $0.1 \mathrm{M} \mathrm{NH}_{4} \mathrm{HCO}_{3}$ at a flow rate of $40 \mathrm{~mL} /$ $\mathrm{h}$, gave $\mathbf{4}$ after freeze-drying as a white powder $(5.3 \mathrm{mg}$, $67 \%) ; R_{f} 0.16(2: 1: 1 \mathrm{AcOH}-1-\mathrm{BuOH}-$ water $) ;[\alpha]_{\mathrm{D}}^{20}-1^{\circ}$ (c $0.3, \mathrm{H}_{2} \mathrm{O}$ ); HRMS of $\mathrm{C}_{46} \mathrm{H}_{81} \mathrm{~N}_{3} \mathrm{O}_{31}$ (M, 1171.485): $[\mathrm{M}+\mathrm{H}]^{+}$found 1172.486 , calcd 1172.492 . For ${ }^{1} \mathrm{H}$ NMR data, see Table 5. 
3.27. 6-Aminohexyl $\beta$-D-galactopyranosyl-( $(\rightarrow 4)-\beta$-Dglucopyranosyl-(1 $\rightarrow 6)$-[ $\beta$-D-galactopyranosyl-(1 $\rightarrow 4)]-2-$ acetamido-2-deoxy- $\beta$-D-glucopyranosyl- $(1 \rightarrow 3)-\beta$-Dgalactopyranosyl-( $1 \rightarrow 4)$ - $\beta$-D-glucopyranosyl-( $1 \rightarrow 6)$-[ $\beta$ D-galactopyranosyl-(1 $\rightarrow 4)]$-2-acetamido-2-deoxy- $\beta$-Dglucopyranoside (2)

To a soln of $4(5.3 \mathrm{mg}, 4.52 \mu \mathrm{mol})$ in aq $50 \mathrm{mM}$ sodium cacodylate buffer $\mathrm{pH} 7.5(500 \mu \mathrm{L})$, containing $5 \mathrm{mM}$ $\mathrm{MnCl}_{2}$, BSA $(0.5 \mathrm{mg})$, and $\mathrm{NaN}_{3}(0.02 \%)$, were added alkaline phosphatase $(10 \mathrm{U})$, UDP-galactose $(8.2 \mathrm{mg}$, $13.4 \mu \mathrm{mol})$, and $\beta$-1,4-galactosyltransferase $(1 \mathrm{U})$. The reaction mixture was incubated for $20 \mathrm{~h}$ at $37^{\circ} \mathrm{C}$, then, water $(100 \mu \mathrm{L})$ was added. UDP-Galactose was removed on a Dowex $1 \times 8\left(\mathrm{Cl}^{-}\right)$column, eluted with water. The eluate was concentrated, and the residue applied on a Bio Gel P-2 column eluted with aq $0.1 \mathrm{M}$ $\mathrm{NH}_{4} \mathrm{HCO}_{3}$ at a flow rate of $40 \mathrm{~mL} / \mathrm{h}$. The appropriate fractions were freeze-dried to give $2(1.5 \mathrm{mg}, 22 \%) ; R_{f}$ 0.07 (2:1:1 AcOH-1-BuOH-water); HRMS of $\mathrm{C}_{58} \mathrm{H}_{101} \mathrm{~N}_{3} \mathrm{O}_{41} \quad(\mathrm{M}, \quad 1495.591):[\mathrm{M}+\mathrm{H}]^{+}$found 1496.614, calcd 1496.598. For ${ }^{1} \mathrm{H}$ NMR data, see Table 6.

\section{Acknowledgements}

We thank H.I.V. Amatdjais-Groenen (Nijmegen University) for measuring the elemental analysis data and $\mathrm{C}$. Versluis (Utrecht University) for recording the HRMS spectra. This work was financially supported by the EU, project BIO CT 95-0138.

\section{References}

1. Hausdorff, W. P.; Bryant, J.; Paradiso, P. R.; Siber, G. R. Clin. Infect. Dis. 2000, 30, 100-121.

2. Sniadack, D. H.; Schwartz, B.; Lipman, H.; Bogaerts, J.; Butler, J. C.; Dagan, R.; Echanizaviles, G.; Lloydevans, N.; Fenoll, A.; Girgis, N. I.; Henrichsen, J.; Klugman, K.; Lehmann, D.; Takala, A. K.; Vandepitte, J.; Gove, S.; Breiman, J. F. Pediatr. Infect. Dis. J. 1995, 14, 503-510.

3. Centers for Disease Control and Prevention. Prevention of pneumococcal disease: recommendations of the Advisory Committee on Immunization Practices (ACIP), $M M W R$ Morb. Mortal. Wkly. Rep. 1997, 46, 1-24.

4. Fine, M. J.; Smith, M. A.; Carson, C. A.; Mutha, S. S.; Sankey, S. S.; Weissfeld, L. A.; Kapoor, W. N. J. Am. Med. Assoc. 1996, 275, 134-141.

5. Doern, G. V.; Brueggemann, A. B.; Huynh, H.; Wingert, E.; Rhomberg, P. Emerg. Infect. Dis. 1999, 5, 757-765.
6. Klugman, K. P. Clin. Microbiol. Rev. 1990, 3, 171196.

7. Robbins, J. B.; Austrian, R.; Lee, C. J.; Rastogi, S. C.; Schiffman, G.; Henrichsen, J.; Mäkalä, P. H.; Broome, C. V.; Facklam, R. R.; Tiesjema, R. H.; Parke, J. C. J., Jr. J. Infect. Dis. 1983, 148, 1136-1159.

8. Butler, J.; Breiman, R.; Campbell, J.; Lipman, H.; Broome, C.; Facklam, R. J. Am. Med. Assoc. 1993, 270, $1826-1831$.

9. Örtqist, Å.; Hedlund, J.; Burman, L.-Å.; Elbel, E.; Höfer, M.; Leinonen, M.; Lindblad, I.; Sundelöf, B.; Kalin, M. Lancet 1998, 351, 399-403.

10. Beuvery, E. C.; Van Rossem, F.; Nagel, J. Infect. Immun. 1982, 37, 15-22.

11. Rennels, M. B.; Edwards, K. M.; Keyserling, H. L.; Reisinger, K. S.; Hogerman, D. A.; Madore, D. V.; Chang, I.; Paradiso, P. R.; Malinoski, F. J.; Kimura, A. Pediatrics 1998, 101, 604-611.

12. Jansen, W. T. M.; Hogenboom, S.; Thijssen, M. J. L.; Kamerling, J. P.; Vliegenthart, J. F. G.; Verhoef, J.; Snippe, H.; Verheul, A. F. M. Infect. Immun. 2001, 69, 787-793.

13. Benaissa-Trouw, B.; Lefeber, D. J.; Kamerling, J. P.; Vliegenthart, J. F. G.; Kraaijeveld, K.; Snippe, H. Infect. Immun. 2001, 69, 4698-4701.

14. Niggemann, J.; Kamerling, J. P.; Vliegenthart, J. F. G. Bioorg. Med. Chem. 1998, 6, 1605-1612.

15. Niggemann, J.; Kamerling, J. P.; Vliegenthart, J. F. G. J. Chem. Soc. Perkin Trans. 1 1998, 3011-3020.

16. Mawas, F.; Niggemann, J.; Jones, C.; Corbel, M. J.; Kamerling, J. P.; Vliegenthart, J. F. G. Infect. Immun. 2002, 70, 5107-5114.

17. Michalik, D.; Vliegenthart, J. F. G.; Kamerling, J. P. J. Chem. Soc. Perkin Trans. 1 2002, 1973-1981.

18. Lindberg, B.; Lönngren, J.; Powell, D. A. Carbohydr. Res. 1977, 58, 177-186.

19. Koeman, F. A. W.; Meissner, J. W. G.; van Ritter, H. R. P.; Kamerling, J. P.; Vliegenthart, J. F. G. J. Carbohydr. Chem. 1994, 13, 1-25.

20. Grundler, G.; Schmidt, R. R. Carbohydr. Res. 1985, 135 , 203-218.

21. Slaghek, T. M.; Nakahara, N.; Ogawa, T.; Kamerling, J. P.; Vliegenthart, J. F. G. Carbohydr. Res. 1994, 255, 6185.

22. Kamerling, J. P. In Streptococcus pneumoniae, Molecular Biology \& Mechanisms of Disease; Thomasz, A., Ed. Pneumococcal polysaccharides: a chemical view; Mary Ann Liebert: New York, 2000; pp 81-114.

23. Zhou, W.; Jennings, H. J. J. Carbohydr. Chem. 1996, 15 , $279-295$

24. Lemieux, R. U.; Driguez, H. J. Am. Chem. Soc. 1975, 97 , 4063-4069.

25. Yoshino, T.; Sadamitsu, M.; Minagawa, M.; Reuter, G.; Schauer, R. Glycoconjugate J. 1988, 5, 377-384. 